\title{
DIGITAL CONTROL BOARD FOR PHASED ARRAY ANTENNA BEAM STEERING IN ADAPTIVE COMMUNICATION APPLICATIONS
}

\author{
A Thesis \\ presented to \\ the Faculty of California Polytechnic State University \\ San Luis Obispo
}

\begin{abstract}
In Partial Fulfillment
of the Requirements for the Degree

Master of Science in Electrical Engineering
\end{abstract}

by

Mayur Bansal

November 2013 
(C) 2013

Mayur Bansal

ALL RIGHTS RESERVED 


\section{COMMITTEE MEMBERSHIP}

TITLE:

Digital Control Board for Phased Array Antenna Beam Steering in Adaptive Communication Applications

AUTHOR:

Mayur Bansal

DATE SUBMITTED: $\quad$ November 2013

COMMITTEE CHAIR: Bryan Mealy, Ph.D.

Associate Professor

Electrical Engineering Department

COMMITTEE MEMBER: Lynne Slivovsky, Ph.D.

Professor

Electrical Engineering Department

COMMitTeE MEMBER: Dennis Derickson, Ph.D.

Department Chair

Electrical Engineering Department 


\begin{abstract}
Digital Control Board for Phased Array Antenna Beam Steering in Adaptive Communication Applications
\end{abstract}

Mayur Bansal

The application of adaptive communication techniques for mobile communications has attracted considerable interest in the last decade. One example of these techniques is spatial filtering through planar antenna array beam forming.

This thesis describes the development of a digital system that adaptively controls a phased array antenna. The radiating structure of the phased antenna array is tetrahedral-shaped and contains four antenna elements on each of its three faces. The overall system comprises of a digital control board with an external computer interface, an RF control board, and the phased antenna array. The RF controls the main lobe direction on the phased array antenna. This thesis describes the design and implementation of the digital control board.

The digital control board's primary responsibilities are implementing interfaces between the external computer and the RF board, which results in two operational modes: the MATLAB graphical user interface (GUI) mode and the adaptive receive mode. The GUI mode allows users to input parameters that provide interactive control of the phased antenna array by interfacing with an external computer and the RF control board. The adaptive receive mode implements an algorithm for an adaptive receive station. This algorithm uses a 58-point scanning technique that locates the maximum receive power direction.

Test results show that the digital control board successfully manages the RF board control voltage with an nominal error of less than $1 \%$, which subsequently 
allows for precise control of the antenna's active face. Additionally, testing of the GUI demonstrated the successful interactive application of various system control parameters. 


\section{ACKNOWLEDGMENTS}

I would like to thank my parents, Meenakshi and Bharat, for encouraging and allowing me to pursue my ambitions. I am also grateful to my entire family for their continual support.

I would like to thank my advisor, Dr. Dean Arakaki, for his guidance and support. I would also like to thanks my project partner Steve Brockhoff for his hard work and help, which made this thesis possible. 


\section{TABLE OF CONTENTS}

LIST OF TABLES Ix

LIST OF FIGURES - x

1 INTRODUCTION 1

2 SYSTEM DESIGN 4

2.1 System Requirements . . . . . . . . . . . . . . 7

2.1.1 GUI Control . . . . . . . . . . . . . . . . 7

2.1.2 Adaptive Receive Mode . . . . . . . . . . . . . . 9

2.2 System Sub-blocks . . . . . . . . . . . . . . . . 10

2.2.1 RF Power Detector . . . . . . . . . . . . . 10

2.2.2 Interrupt Feedback Loop . . . . . . . . . . . . . . . . . 10

3 RF POWER DETECTOR 12

3.1 LT-5534 . . . . . . . . . . . . . . . . . . . . . . 14

3.2 LT-5538 . . . . . . . . . . . . . . . . . . 16

3.3 Log-Amp Power Detector Analysis . . . . . . . . . . . . . . . 18

4 INTERRUPT FEEDBACK LOOP 19

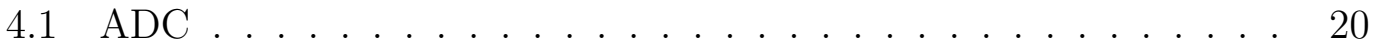

4.2 Interrupt . . . . . . . . . . . . . . . . . 23

$4.3 \mathrm{DAC} \ldots \ldots \ldots \ldots \ldots \ldots \ldots \ldots$

4.4 Comparator ........................ 27

5 RF SYSTEM INTEGRATION 29

5.1 Phase Shifters . . . . . . . . . . . . . . . . . . . . 29

5.2 RF Single Pole Double Throw (SPDT) Switch . . . . . . . . . . . 34

5.3 RF Single Pole Triple Throw (SP3T) Switch . . . . . . . . . . . . 35

5.4 Voltage Control Oscillator (VCO) Control Line . . . . . . . . . 36 
6 PRINTED CIRCUIT BOARD DESIGN 39

6.1 Schematic . . . . . . . . . . . . . . . . . . 39

6.2 Layout . . . . . . . . . . . . . . . . . . . 40

7 GRAPHICAL USER INTERFACE (GUI) CONTROL SYSTEM 42

7.1 GUI Inputs and Features . . . . . . . . . . . . . . . . 43

7.1.1 Drop Down Menus ............... 43

7.1 .2 Edit Text ................... 47

7.1.3 Push Buttons . . . . . . . . . . . . . . . . 49

7.1.4 Additional Figure Checkboxes . . . . . . . . . . . . 50

7.2 Communication Protocol . . . . . . . . . . . . . . . . 51

7.3 Test and Results . . . . . . . . . . . . . . . 53

8 ADAPTIVE RECEIVE MODE 58

8.1 Adaptive Receive Algorithm . . . . . . . . . . . . . . . . . . . 58

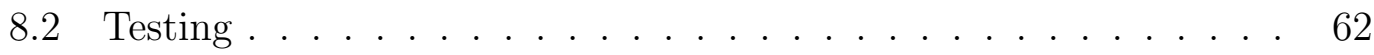

9 CONCLUSIONS AND FUTURE WORK 65

$\begin{array}{ll}\text { BIBLIOGRAPHY } & 67\end{array}$

$\begin{array}{ll}\text { APPENDICES } & 69\end{array}$

A 2013 IEEE APS Student Design Challenge: Reconfigurable Antennas 69

B Contest Proposal . . . . . . . . . . . . . . . . 72

C LT 5534 Demo Board Schematic . . . . . . . . . . . . . . 75

D LT 5538 Demo Board Schematic . . . . . . . . . . . . . . . 77

E Microcontroller Code . . . . . . . . . . . . . . 79

F PCB Schematic . . . . . . . . . . . . . . . . 90

G PCB Layout . . . . . . . . . . . . . . . . . 96

$\mathrm{H}$ Bill Of Materials .................... 98

I Block Diagrams . . . . . . . . . . . . . . 100 


\section{LIST OF TABLES}

2.1 16-Pin IDC Pin-out Between Digital Control Board and RF Board 6

4.1 ADC Conversion Time . . . . . . . . . . . . . . . . . . 22

4.2 ADC Configuration . . . . . . . . . . . . . . . . . 23

4.3 Interrupt Configuration . . . . . . . . . . . . . . . . . 23

4.4 DAC Command Codes . . . . . . . . . . . . . . 26

4.5 DAC Address Codes . . . . . . . . . . . . . . . . . 26

5.1 Truth Table for SPDT, $V_{H I G H}=2 \mathrm{~V}$ to $5 \mathrm{~V}, V_{L O W}=0 \mathrm{~V}$ Insertion Loss $=0.5 \mathrm{~dB}$, and Isolation $=22 \mathrm{~dB} \ldots \ldots . . . . . . .34$

5.2 SPDT, Antenna Mode Truth Table . . . . . . . . . . . . . . . . 35

5.3 Truth Table for HMC245QS16 and the Active Antenna Face . . . 36

5.4 VCO Tuning Circuit Result . . . . . . . . . . . . . . . . . . . 38

7.1 Hexadecimal Notation for ASCII Decimal Characters to 8-bit Integers ........................... 52

7.2 GUI Test Result . . . . . . . . . . . . . . . . . . . . . . . 54

8.1 Test Data for Adaptive Receive Mode Test Procedure . . . . . . . 63 


\section{LIST OF FIGURES}

1.1 A Radiation Diagram with Main Lobe . . . . . . . . . . . . 2

2.1 System Block Diagram . . . . . . . . . . . . . . . . . 4

2.2 Tetrahedron Antenna Model . . . . . . . . . . . . . . . . . 5

2.3 Digital Control Board Block Diagram . . . . . . . . . . . 5

2.4 IDC connector Pin Positions . . . . . . . . . . . . . . . . 6

2.5 Screen shot of MATLAB GUI . . . . . . . . . . . . . 8

2.6 Adaptive RX, Example System Configuration . . . . . . . . . 9

2.7 Inner Feedback Loop Block Diagram _ . . . . . . . . . . . 11

3.1 DC log-amp BJT implementation . . . . . . . . . . . . . 13

3.2 Demo Board DC748A . . . . . . . . . . . . . . . . . . . 14

$3.3 \quad$ LT-5534 log-amp Characteristics . . . . . . . . . . . . . . . 15

3.4 Demo Board DC1120A . . . . . . . . . . . . . . . . . 16

3.5 LT-5538 Output characteristics . . . . . . . . . . . . . 17

4.1 Analog to Digital Converter Timing Diagram for Single Conversion Mode . . . . . . . . . . . . . . . . . . . . . 22

$4.2 \quad$ LTC2656 DAC Load Sequence . . . . . . . . . . . . . . . 25

4.3 LM339 Schematic Diagram . . . . . . . . . . . . . . . . . . . 28

5.1 JSPHS-2484+ Phase Shifter, Interpolated Data . . . . . . . 30

5.2 Operational Amplifier in Non-Inverting Configuration . . . . . . 30

5.3 LTSpice Simulation Schematic, LT6005 Non-Inverting Gain OpAmp Circuit . . . . . . . . . . . . . . . . . 31

5.4 LTSpice Simulation results, LT6005 Op-amp Non-Inverting Gain Circuit . . . . . . . . . . . . . . . . . 32 
5.4 LTSpice Simulation results, LT6005 Op-amp Non-Inverting Gain Circuit . . . . . . . . . . . . . . . . . 33

5.5 Monte Carlo Analysis for Amplification Circuit, Expanded View . 33

5.6 AS179-92LF, SPDT, Pin-out and Block Diagram . . . . . . . 34

5.7 HMC245QS16 SP3T RF Switch Pin-out and Functional Diagram 35

5.8 JTOS-2700V, VCO, datasheet characteristic . . . . . . . . . 37

5.9 VCO tuning Schematic, R5 is the tunable resistor . . . . . . 38

6.1 PCB layout $\ldots \ldots \ldots \ldots \ldots \ldots \ldots \ldots \ldots \ldots \ldots \ldots \ldots$

7.1 RS-232, 9-pin D-sub connector pinout . . . . . . . . . . 42

7.2 MATLAB GUI main screen . . . . . . . . . . . . . . . 43

7.3 MATLAB GUI screen with Face drop down menu . . . . . . . . 44

7.4 MATLAB GUI Screen with Mode Drop-Down Menu . . . . . . . . 45

7.5 TRENDnet TU-S9, USB to RS-232 Converter Cable . . . . . . 45

7.6 Device Manager screen shot with COM port highlighted . . . . 46

7.7 MATLAB GUI screen with COM drop down menu $\ldots \ldots$. . . 47

7.8 Antenna Radiation Pattern Coordinate system . . . . . . . . . . 48

7.9 MATLAB GUI Screen Shot, $\theta$ and $\phi$ Control Variables . . . . . 49

7.10 Phase Shifter JSPHS-2484+ Interpolated Data . . . . . . . 50

7.11 Checkbox Selected Images, with $\theta=30^{\circ}$ and $\phi=45^{\circ} \ldots \ldots$

7.12 RS-232 Frame . . . . . . . . . . . . . . . . . . 51

7.13 Communication Packet Frame _. . . . . . . . . . . 52

7.14 Microcontroller, MATLAB GUI Mode Flowchart . . . . . . . 53

7.15 Status LED's for Mode and Face value . . . . . . . . . . . . 55

7.16 GUI Test Measured Radiation Patterns . . . . . . . . . . . . 56

7.16 GUI Test Measured Radiation Patterns . . . . . . . . . . . . 57

8.1 Adaptive Receive scan plot . . . . . . . . . . . . . . . . . . 59

8.2 Raster Scan Pattern . . . . . . . . . . . . . . . . 60

8.3 Adaptive Receive Flowchart . . . . . . . . . . . . . . . . . . 61

8.4 ISR Flowchart . . . . . . . . . . . . . . . . . . . . . 62 
I.1 Schottky Diode Chip Model . . . . . . . . . . . . . . . . . . 100

I.2 ATmega2560 Block Diagram . . . . . . . . . . . . . . . . 101

I.3 Analog to Digital Converter Block Schematic . . . . . . . . . . . . 102

I.4 LTC2656 Block Diagram . . . . . . . . . . . . . . . . . 103 


\section{CHAPTER 1}

\section{INTRODUCTION}

An adaptive communication system was proposed as a contest entry to the $4^{\text {th }}$ IEEE Antenna Design Challenge, sponsored by the Antenna and Propagation Society (AP-S). This thesis describes an adaptive communication system, which was developed as a contest entry. The main challenge of the contest was to develop an "antenna system with reconfigurable antenna elements that can adapt to different propagation conditions (e.g. line-of-sight versus non-line-of-sight)." The IEEE contest rules are shown in Appendix A. The two page proposal for the design challenge is shown in Appendix B.

A phased adaptive antenna array was proposed for the contest entry. Adaptive phased array antennas can change their radiation pattern electronically or mechanically. In electrically configurable radiation pattern antenna, elements have a relative phase difference while the antenna elements remain stationary. Whereas in mechanical reconfigurable radiation patterns, antenna elements are placed relative to each other but the antenna elements are movable. A tetrahedral structure with four patch antennas on each face of the antenna is developed, as shown in Figure 2.2. Only one face is active at any given moment. A relative phase difference in the four patch antenna changes the radiation pattern's main lobe direction, which is referred to as beam steering. The main lobe of an antenna radiation pattern contains the maximum power. Figure 1.1 shows a main lobe for a radiation pattern. 


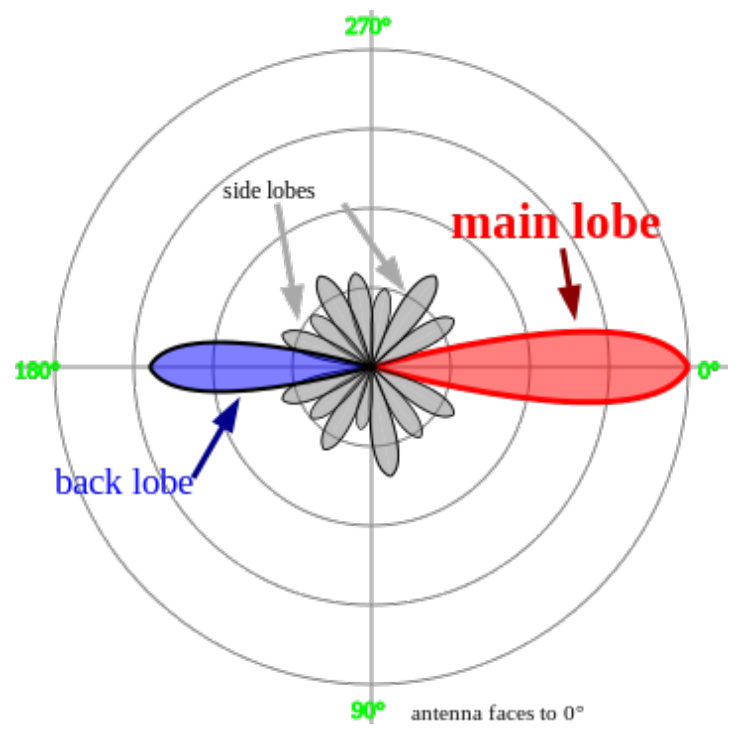

Figure 1.1: A Radiation Diagram with Main Lobe

The goal of this thesis is to design and implement a Digital Control System to control the Electronically-Steered Phased Array Antenna described in [1]. The controllable fields for the RF front-end system include the active antenna face, two communication modes (transmit or receive), and control voltages for a phase shifter that sets the main beam direction. The digital control board components include:

\section{RF Power Detector}

The RF power detector measures the power received from the RF system. The received power is interpreted by the microcontroller using an ADC, which is further explained in Chapter 8.

\section{Microcontroller}

A microcontroller provides a communication interface between the computer and RF system; see Chapter 7 for additional details. The microcontroller also executes adaptive communication algorithms, as explained in 
Chapter 8.

\section{Digital Peripherals}

The digital peripherals include a digital-to-analog converter (DAC), a comparator, and an analog-to-digital converter (ADC). The ADC is an internal peripheral of the microcontroller. The DAC output voltages control the phase shifters (via an SPI bus) to steer the antenna beam. The ADC measures received power by sampling the RF power detector output voltage. The comparator checks the receive power level against a reference level set by the DAC; this level is the previously received maximum power and is discussed further in Chapter 4. A feedback loop is implemented using an $\mathrm{ADC}, \mathrm{DAC}$ and a comparator. The feedback loop improves the time required to find the receive direction with maximum power. The feedback loop is explained in Chapter 4. 


\section{CHAPTER 2}

\section{SYSTEM DESIGN}

An AVR-based microcontroller serves as the system controller. The AVR model is an 8-bit Reduced Instruction Set Computer (RISC) single-chip microcontroller developed by Atmel. Figure 2.1 shows the system basic block diagram.

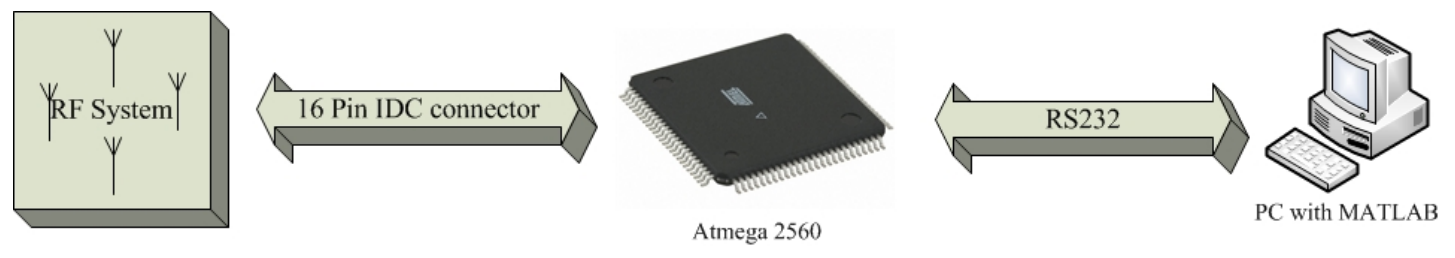

Figure 2.1: System Block Diagram

The adaptive antenna system is a tetrahedron shaped structure (Figure 2.2) with $2 \mathrm{X} 2$ patch arrays on each of the three faces. Only one face is active at a time. The system is half duplex in that it can both transmit and receive, but not simultaneously. 


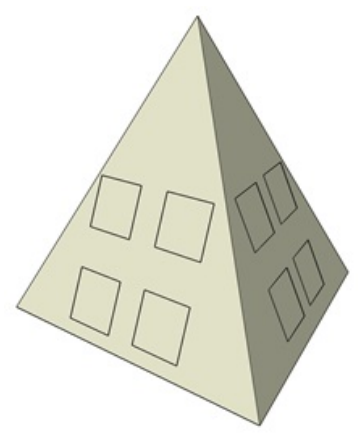

Figure 2.2: Tetrahedron Antenna Model

Figure 2.3 is the level-1 block diagram for the system. The block diagram shows the main system blocks and the associated data flow. The yellow blocks correspond to the RF components on the board. The purple blocks correspond to the analog system on the board. The pink blocks are the USART support components.

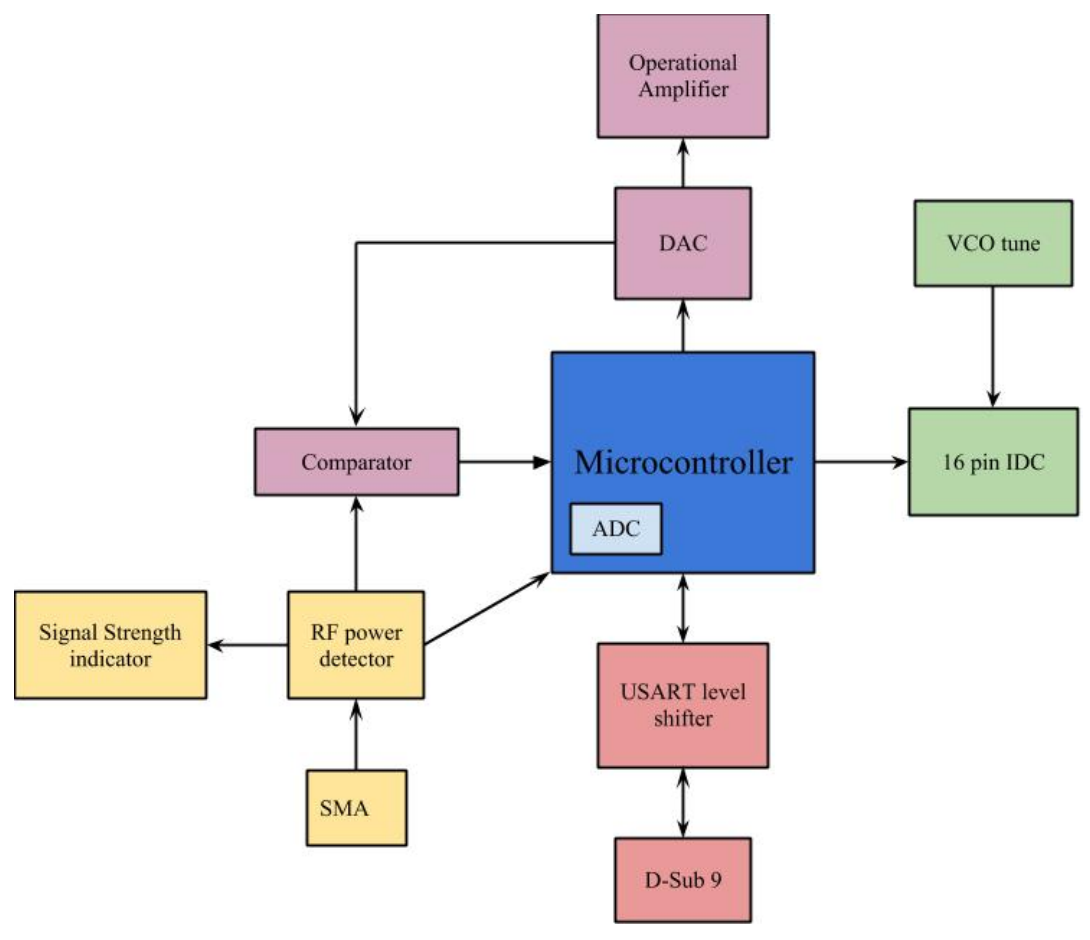

Figure 2.3: Digital Control Board Block Diagram 
Table 2.1: 16-Pin IDC Pin-out Between Digital Control Board and RF Board

\begin{tabular}{|r||r|l|}
\hline PIN \# & Control Line & Function \\
\hline 1 & GND & Ground Connection \\
\hline 2 & GND & Ground Connection \\
\hline 3 & CTRL1 & Unused \\
\hline 4 & CTRL0 & Unused \\
\hline 5 & SPDT0 & Control line for Single pole double throw \\
\hline 6 & PS1 & Control Voltage Phase Shifter 1 \\
\hline 7 & SPDT1 & Control line for Single pole double throw \\
\hline 8 & PS2 & Control Voltage Phase Shifter 2 \\
\hline 9 & SP3T0 & Control line for Single pole triple throw \\
\hline 10 & PS3 & Control Voltage Phase Shifter 3 \\
\hline 11 & SP3T1 & Control line for Single pole triple throw \\
\hline 12 & PS4 & Control Voltage Phase Shifter 4 \\
\hline 13 & GND & Ground Connection \\
\hline 14 & VCO & Control Voltage for the Voltage Control oscillator \\
\hline 15 & GND & Ground Connection \\
\hline 16 & GND & Ground Connection \\
\hline & & \\
\hline 16 & &
\end{tabular}

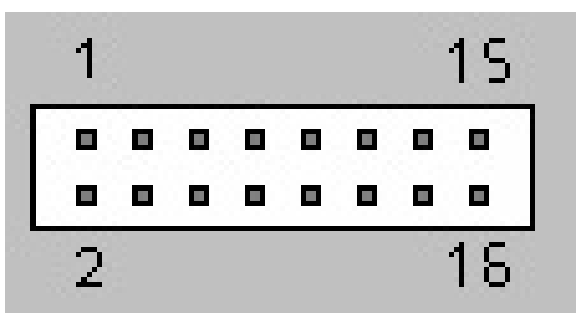

Figure 2.4: IDC connector Pin Positions 
The control board and RF system communicate via a 16-pin insulation displacement connector (IDC). The IDC pinout is defined in Table 2.1 and pin positions are shown in Figure 2.4. System requirements for the digital control board include:

\section{Graphical User Interface (GUI) Controlled System}

A MATLAB-based GUI tests the system's beam steering. The GUI communicates with the digital control board via a Universal Asynchronous Receiver/Transmitter (USART) using a RS-232 protocol. All antenna system variables are defined through the GUI; see Chapter 7.

\section{Adaptive RX}

An adaptive receive station directs the system's radiation pattern in the maximum receive power direction. The scan algorithm for finding this direction is explained in Chapter 8.

\subsection{System Requirements}

\subsubsection{GUI Control}

The first project goal is to interface the system with the MATLAB GUI that defines phase shifter voltages, mode, and face; see Figure 2.5. 


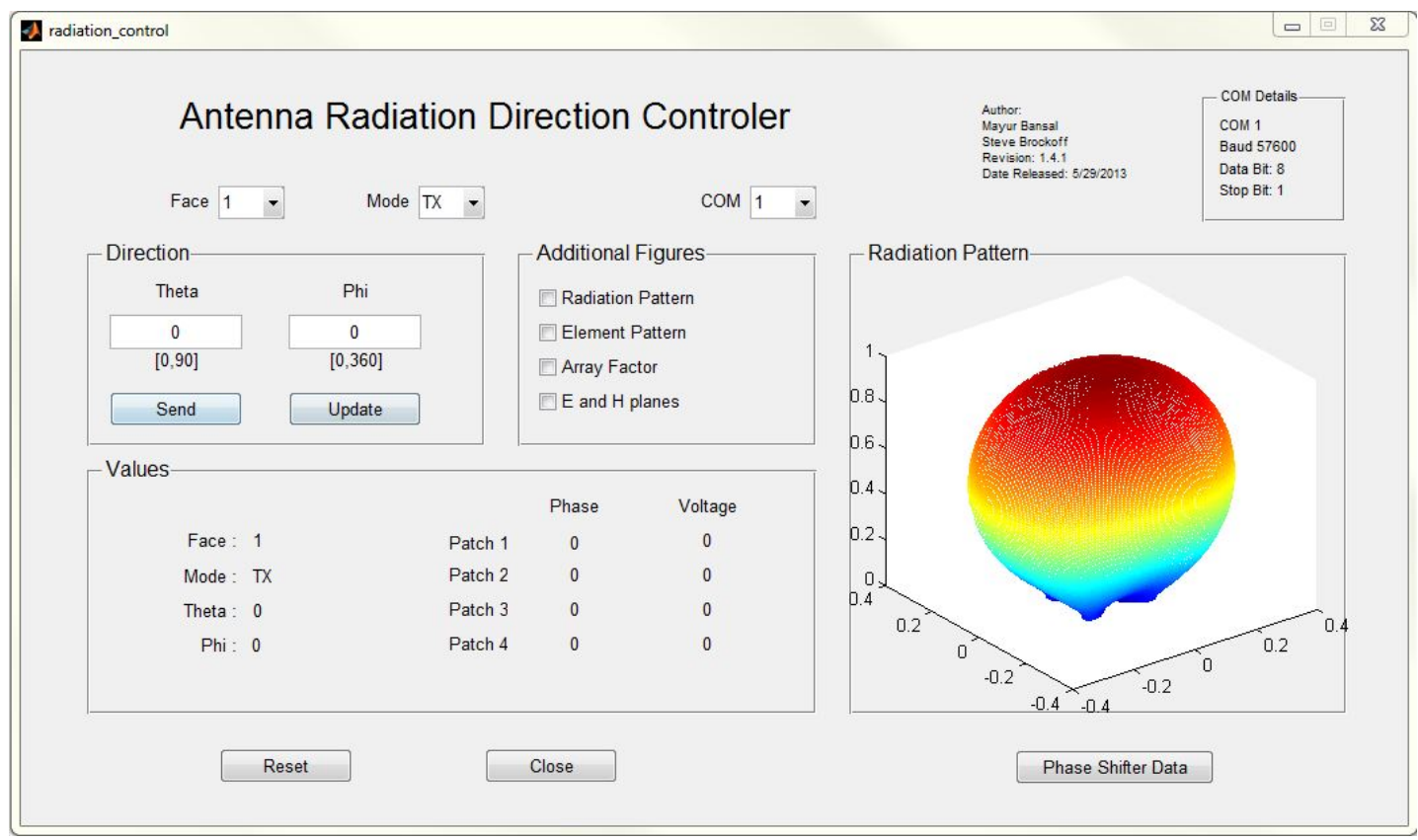

Figure 2.5: Screen shot of MATLAB GUI

\section{Phase Shifter Voltages}

Voltage controlled phase shifters in the RF front-end are used to direct the radiation pattern's main beam [1].

\section{Mode}

The system has two modes: transmit and receive. Single pole double throw (SPDT) switches control the modes; see Section 5.2.

\section{Face}

The tetrahedral antenna structure has three faces. The active face is controlled using single pole triple throw (SP3T) switches; see Section 5.3.

A USART is used for MATLAB GUI and system communications. The Voltage Controlled Oscillator (VCO) output frequency is controlled by an external potentiometer for fine adjustments. Detailed GUI information is provided in Chapter 7. 


\subsubsection{Adaptive Receive Mode}

The second system goal is to operate as an adaptive receive station, which tracks a transmitting signal's location. A scan algorithm determines the maximum power direction and is described in Chapter 8. Figure 2.6 shows two 'RX antenna' placements and how the radiation patterns adapt to the optimum direction. Additional information appears in Chapter 8 .

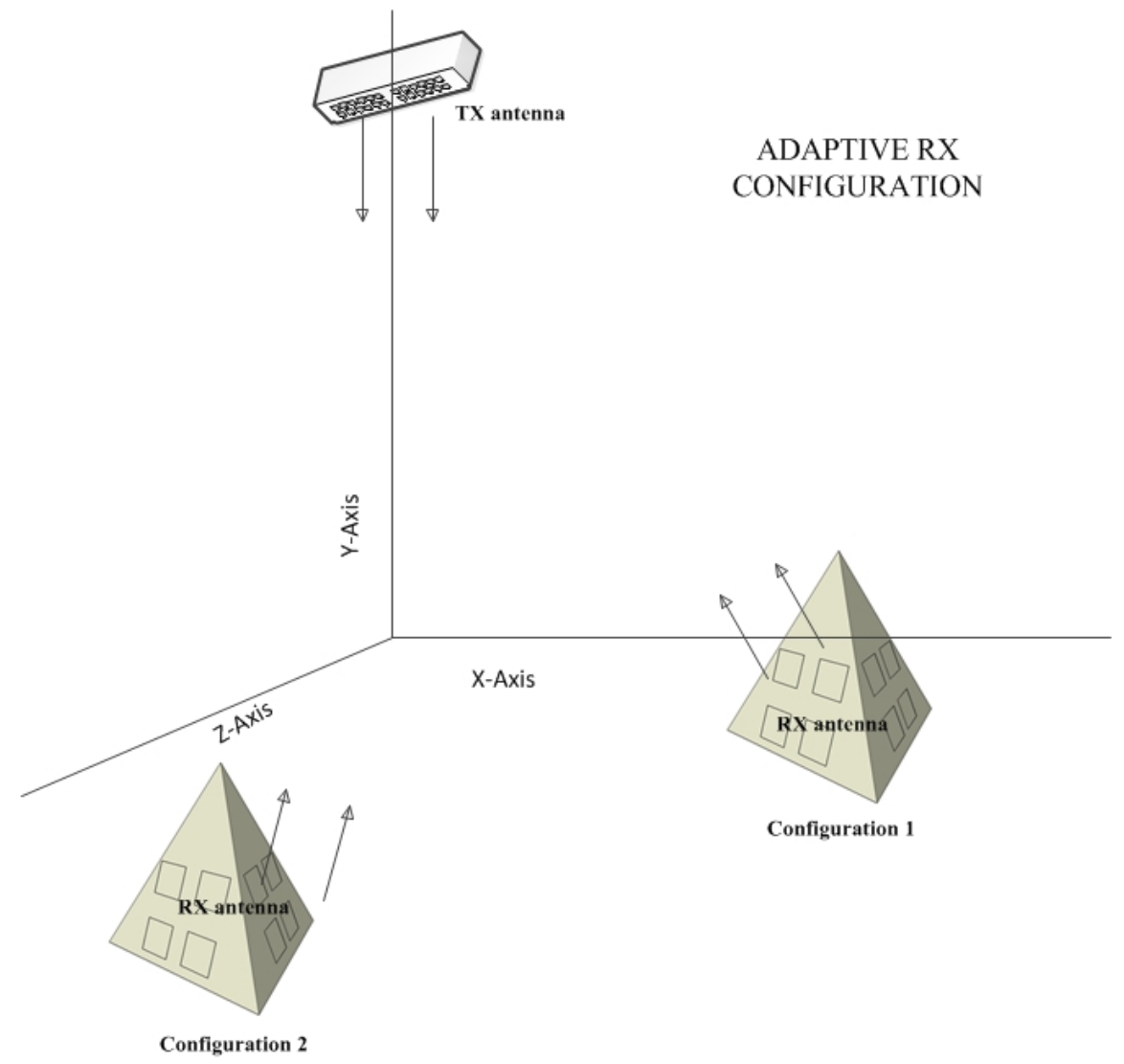

Figure 2.6: Adaptive RX, Example System Configuration 


\subsection{System Sub-blocks}

\subsubsection{RF Power Detector}

The RF power detector converts the received $\mathrm{RF}$ power to a $\mathrm{DC}$ voltage. Most RF power detectors are Schottky diode-based topologies; they exhibit an exponential

relation between input power and output voltage, $V_{\text {out }}(V) \propto e^{P_{\text {in }}}(W)$. Another common implementation is a log-amp based topology, which exhibits a linear relationship between input power and output voltage, $V_{\text {out }}(V) \propto P_{\text {in }}(W)$. RF power detector information appears in Chapter 3.

\subsubsection{Interrupt Feedback Loop}

The block diagram of the Interrupt Feedback Loop is shown in Figure 2.7. The Interrupt Feedback Loop improves system efficiency by reducing the number of required ADC samples at every step. The ADC samples only when hardware interrupts are triggered; i.e.: when a power level that exceeds the previous maximum is detected. When an interrupt is triggered, the received power is saved in memory as the new maximum value. The feedback loop includes a comparator, the RF power detector and the microcontroller. The microcontroller uses a DAC to set the comparator's reference level, which represents the previous maximum received power. Interrupt feedback loop information is provided in Chapter 4. 


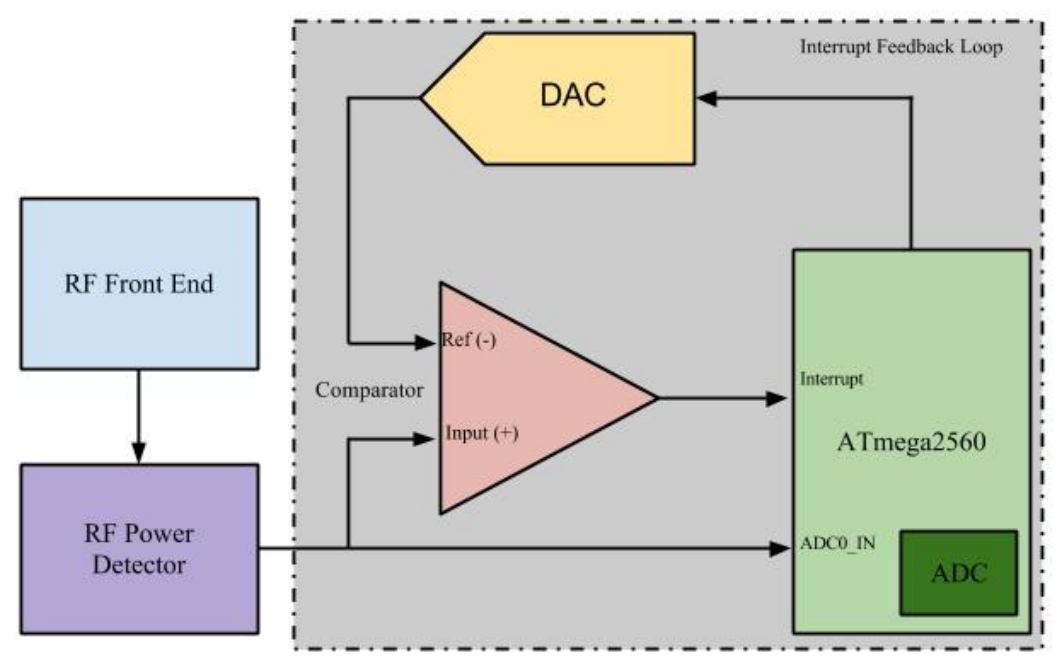

Figure 2.7: Inner Feedback Loop Block Diagram 


\section{CHAPTER 3}

\section{RF POWER DETECTOR}

An RF power detector monitors the RF circuit's output and develops a DC voltage proportional to the received power in Watts [2]. Equation 3.1 shows power in decibels relative to $1 \mathrm{~mW}(\mathrm{dBm})$, commonly used by $\mathrm{RF}$ and wireless engineers.

$$
P(d B m)=10 \log P(m W)
$$

An operational amplifier (op-amp) with a PN junction diode as negative feedback is a classical implementation for a log-amp [3]. The logarithmic relationship between diode voltage and current yields a linear relationship between input power (dB scale) and output voltage (linear voltage scale). A bipolar junction transistor (BJT) generates the logarithmic $I$ vs $V$ relation in place of a diode. Figure 3.1 shows implementation examples for the single stage NPN and PNP log-amp. Equation 3.2 defines RF power detector output voltage $\left(V_{\text {out }}\right)$ as a function of the input power $\left(I_{i n}\right)$.

$$
V_{\text {out }}=\frac{k T}{q} \ln \frac{I_{C}}{I_{S}} \approx \frac{k T}{q} \ln \frac{I_{I n}}{I_{S}}
$$




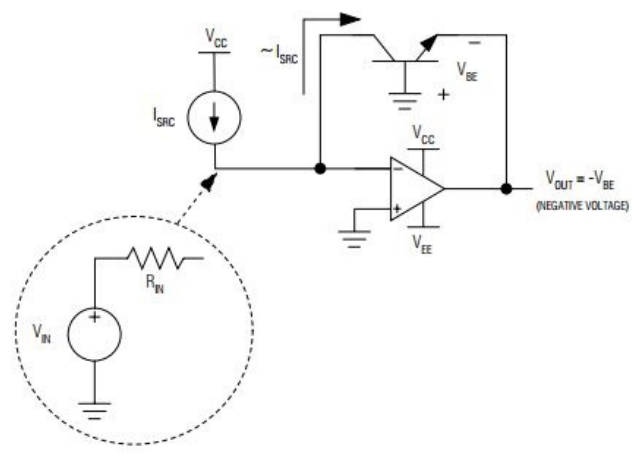

(a) Basic NPN log-amp

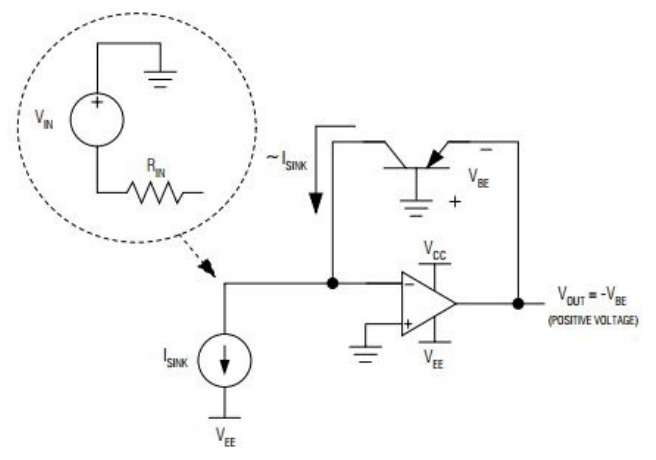

(b) Basic PNP log-amp

\section{Figure 3.1: DC log-amp BJT implementation}

Two Linear Technology Corp. (LTC) log-amps were compared to maximize dynamic range and output voltage slope. Log-amp datasheet characteristics include:

1. LT 5534

- RF Frequency Range: $50 \mathrm{MHz}$ to $3 \mathrm{GHz}$

- Linear Dynamic Range: 60dB

- Supply Voltage: $2.70 \mathrm{~V}$ to $5.25 \mathrm{~V}$

- Low Supply Current: $7 \mathrm{~mA}[4]$

2. LT 5538

- RF Frequency Range: $40 \mathrm{MHz}$ to $3.8 \mathrm{GHz}$

- Linear Dynamic Range: 75dB

- Supply Voltage: $3.00 \mathrm{~V}$ to $5.25 \mathrm{~V}$

- Low Supply Current: 29mA [5] 
The log-amps were compared to the required dynamic range, 60dB, and the output slope of the linear $V_{\text {out }}(V)$ vs input power $(\mathrm{dBm})$ relationship, > $20 \mathrm{mV} / \mathrm{dB}$. Comparison results are shown in Section 3.3.

\section{$3.1 \quad$ LT-5534}

The LT-5534 is a monolithic RF power detector capable of measuring RF signals over a dynamic range of $-63 \mathrm{dBm}$ to $3 \mathrm{dBm}$ at $2.5 \mathrm{GHz}$ [6]. A Linear Technology Corp log-amp demo board DC748A enabled LT-5534 characterization, as shown in Figure 3.2 The demo board's schematic is shown in Appendix C [7].

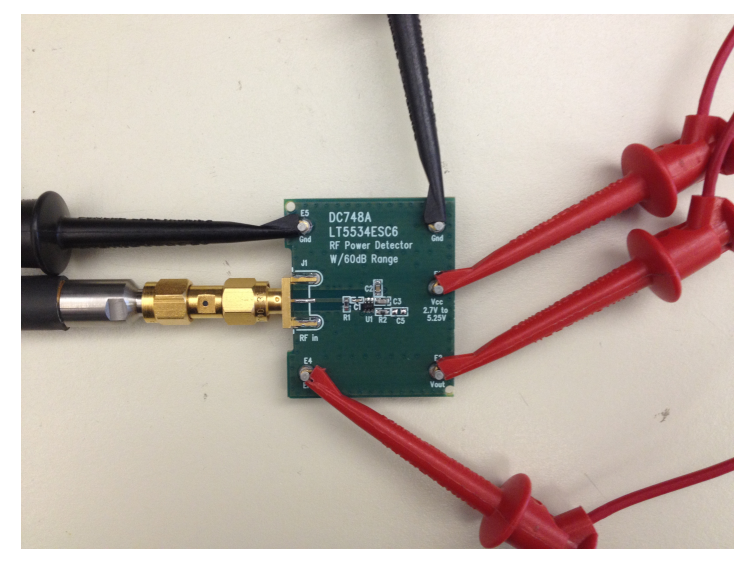

Figure 3.2: Demo Board DC748A

The log-amp, LT-5534, output characteristics were compared to datasheet values. Figure 3.3a shows measured output voltage $V_{\text {out }}(V)$ vs Input Power $(\mathrm{dBm})$ at $2.4 \mathrm{GHz}$, Figure $3.3 \mathrm{~b}$ shows the same plot provided by the datasheet. The measured output slope is $0.0373 \mathrm{~V} / \mathrm{dB}$ compared to $0.0366 \mathrm{~V} / \mathrm{dB}$ in the datasheet [6]. The minimum detectable signal power is $-60 \mathrm{dBm}$. 


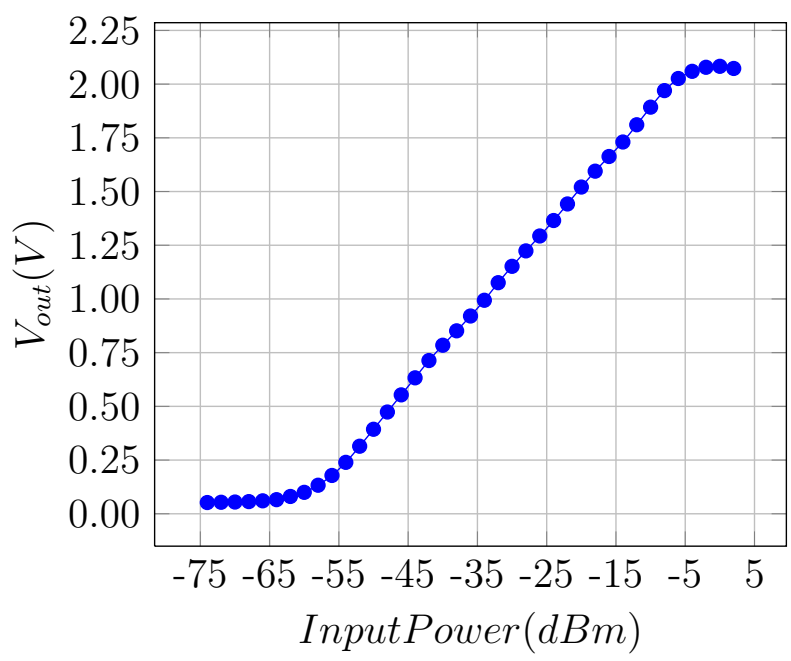

(a) Measured

\section{Output Voltage and Linearity Error} vs Input Power

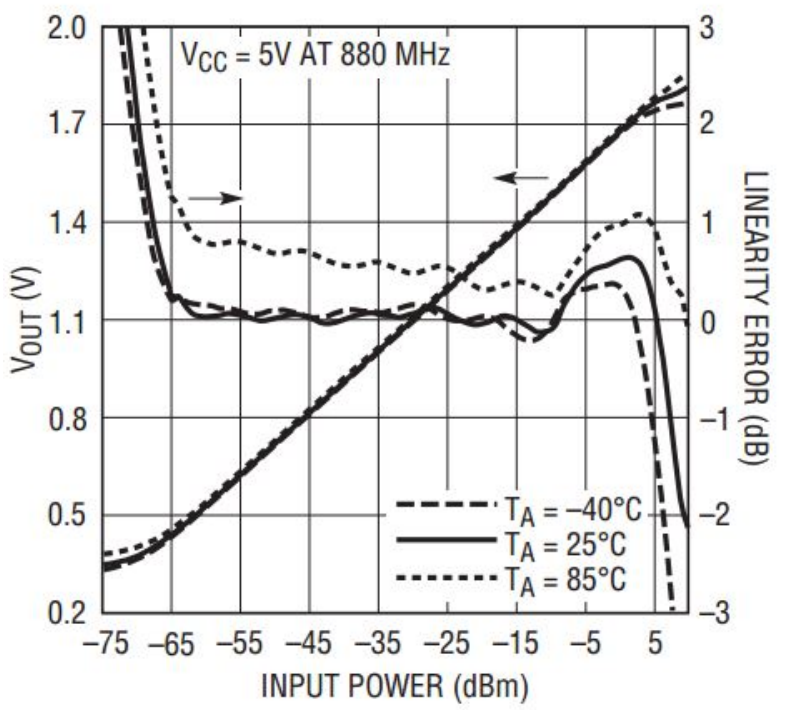

(b) Datasheet

Figure 3.3: LT-5534 log-amp Characteristics 


\section{$3.2 \quad$ LT-5538}

The LT-5538 is a monolithic RF power detector capable of measuring RF signals over a dynamic range of $-72 \mathrm{dBm}$ to $10 \mathrm{dBm}$ at $2.7 \mathrm{GHz}[8]$. The demo board DC1120A, Figure 3.4 schematic can be found in Appendix D [9].

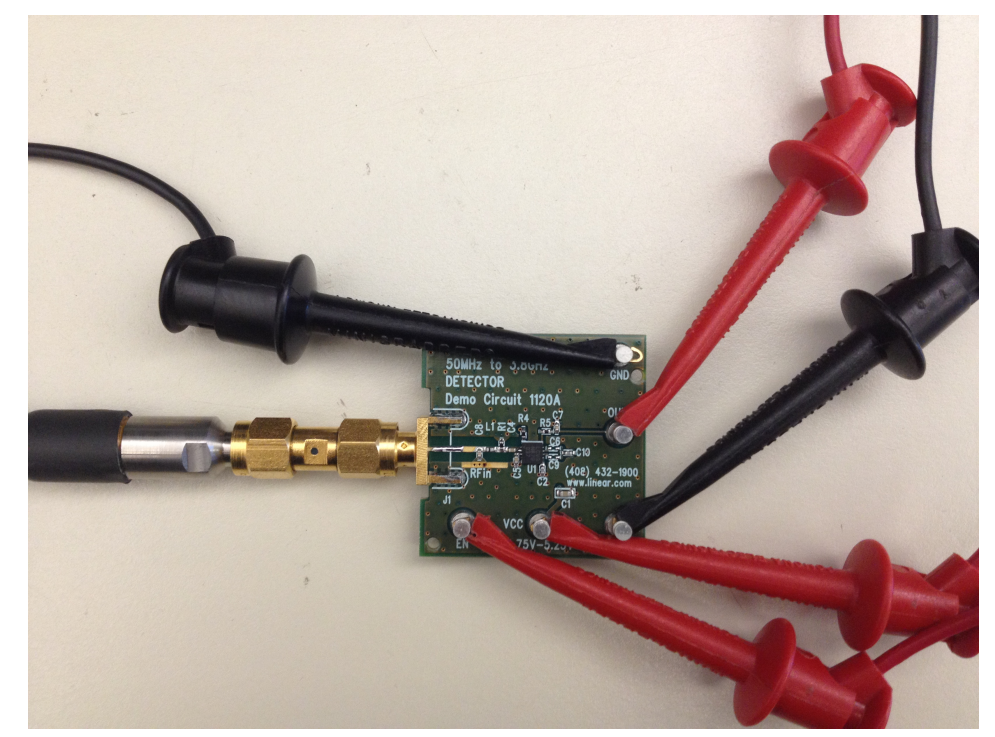

Figure 3.4: Demo Board DC1120A

The LT-5538 log-amp`s output characteristics are compared to datasheet values. Figure 3.5a shows the measured output voltage $V_{\text {out }}(V)$ vs. Input Power $(\mathrm{dBm})$ at $2.4 \mathrm{GHz}$. Figure $3.5 \mathrm{~b}$ shows the same plot provided by the datasheet. The measured output slope is $0.0180 \mathrm{~V} / \mathrm{dB}$ compared to the datasheet value of $0.0176 \mathrm{~V} / \mathrm{dB}[8]$. The minimum detectable signal power is $-68 \mathrm{dBm}$. 


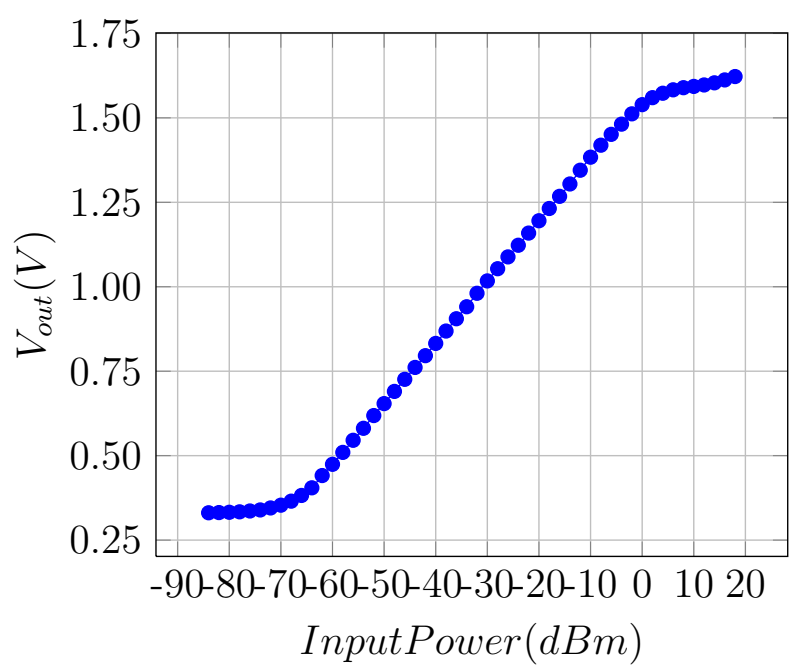

(a) Measured

Output Voltage and Linearity Error vs Input Power

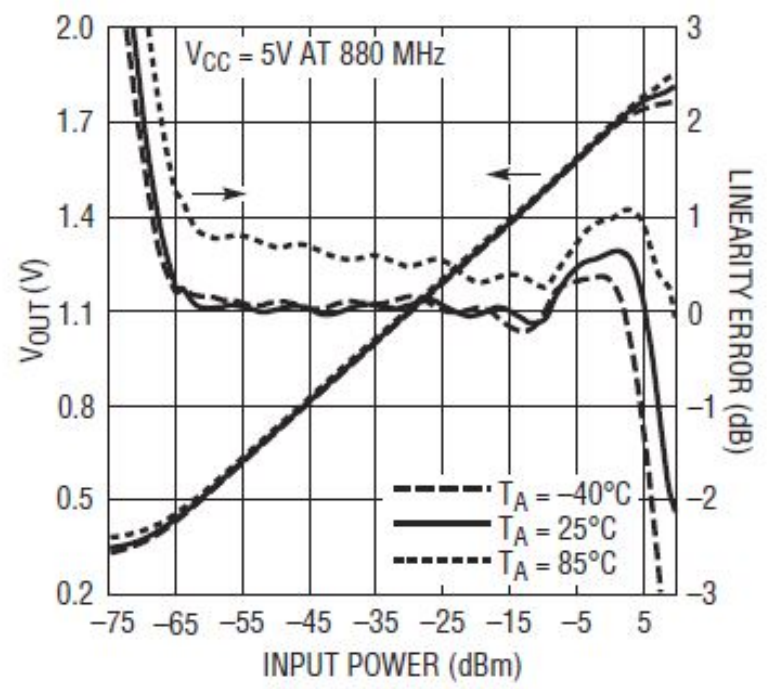

(b) Datasheet

Figure 3.5: LT-5538 Output characteristics 


\subsection{Log-Amp Power Detector Analysis}

From the previous sub-sections, the LT-5534 and LT-5538 have output slopes of $37.3 \mathrm{mV} / \mathrm{dB}$ and $18.0 \mathrm{mV} / \mathrm{dB}$, respectively. For an 8-bit ADC with reference voltage of $5 \mathrm{~V}$, a quantum is $19.6 \mathrm{mV}$, as shown in Equation 3.3.

$$
\text { quanta }=\frac{5 \mathrm{~V}}{2^{8}-1}=19.6 \mathrm{mV}
$$

Thus the LT-5534 is the best option, since the dynamic range satisfies the required range $(-60 \mathrm{dBm}$ to $-30 \mathrm{dBm})$ and the output slope is greater than one quanta $(19.6 \mathrm{mV} / \mathrm{dB})$. 


\section{CHAPTER 4}

\section{INTERRUPT FEEDBACK LOOP}

Microcontrollers are widely used in communication applications. The AT-mega2560 AVR architecture [12], microcontroller is the main system controller for the system. Key features include:

- Flash : 256 Kbytes $(\mathrm{kB})$

- Static Random Access Memory (SRAM): 8 kB

- Maximum Operating Frequency: $16 \mathrm{MHz}$

- Serial Peripheral Interface (SPI): 5 channels

- Analog-to-Digital Converter: 16 channels, 10-Bit Resolution

- Real Time Clock (RTC)

Flash memory stores the boot loader and firmware. The boot loader is a program that loads the firmware, or runtime environment, for the processor following self-test completion. SRAM microcontroller memory used for system variable declaration and firmware operation. An algorithm with multiple subroutines

requires an extensive $(>128 \mathrm{kB})$ flash memory (see Section 8.1 for algorithm). Status variables are required for each algorithm branch; thus, greater than $2 \mathrm{kB}$ of SRAM must be available. Many current digital peripherals use a communication protocol (SPI, CAN, I2C, USART, etc.) for intercommunication. SPI and 
USART are the two communication buses used in this system. ADC converts the $\mathrm{RF}$ peak detector DC output voltage into a digital value. A $2 \%$ output accuracy and resolution greater than eight bits is required to capture a $0.25 \mathrm{dBm}$ change in received power level. Real time clock (RTC) compatibility is preferred for systems synchronization. Figure I.2 (Appendix I) shows the microcontroller's block diagram.

The inner feedback loop (Figure 2.7) consists of an ADC, DAC, and a comparator. The DAC sets the reference input to the comparator. The peak detector output is connected to both the positive input terminal of the comparator and the microcontroller's ADC input.

When the peak detector output is greater than the comparator's reference voltage, the comparator output changes to logic high, triggering a microcontroller interrupt. The microcontroller is notified that a new maximum power has been received and is sampled by the microcontroller's ADC. In this case, a new reference value is set by the DAC, and microcontroller stores the face and phase counters (see Section 8.1). This loop eliminates redundant power detector output voltage sampling in each algorithm step. Only voltage levels greater than the previously received maximum power are sampled. Lock time is defined as the time required to find the optimal receive direction.

\subsection{ADC}

The microcontroller's ADC samples the power detector's output voltage. Figure I.3 (Appendix I) shows the microcontroller's ADC block schematic. Key features include:

- 10-bit resolution 
- $13 \mu \mathrm{s}-260 \mu \mathrm{s}$ conversion time

- $0 V-V_{C C} \mathrm{ADC}$ input voltage range, $V_{C C}=5 \mathrm{~V}$

- Free running or single conversion mode

The ATmega2560 uses a 10-bit successive approximation ADC that can be configured as either a single-ended or a differential input. A single-ended ADC is preferable since negative voltages are not sampled. Equation 4.1 defines the digital output for a single-ended conversion, where $V_{I N}$ is the voltage at the selected input and $V_{R E F}$ is the selected voltage reference.

$$
\text { ADCout }=\text { floor }\left(\frac{V_{I N} \cdot 1024}{V_{R E F}}\right)
$$

The 10-bit ADC resolution results in a quanta of $\left(V_{C C}=5 \mathrm{~V}\right)$ :

$$
\text { quanta }=\frac{V_{C C}}{2^{n}-1}=\frac{5 \mathrm{~V}}{2^{10}-1}=4.9 \mathrm{mV}
$$

The ADC is configured in single conversion mode since samples are recorded only upon interrupt triggers. As specified in Table 4.1, the first conversion requires 25 clock cycles, while subsequent conversions require 13 clock cycles. 
Table 4.1: ADC Conversion Time

\begin{tabular}{|l|l|l|}
\hline Condition & $\begin{array}{l}\text { Sample \& Hold } \\
\text { (Cycles from Start } \\
\text { of Conversion) }\end{array}$ & $\begin{array}{l}\text { Conversion Time } \\
\text { (Cycles) }\end{array}$ \\
\hline First conversion & 13.5 & 25 \\
\hline $\begin{array}{l}\text { Subsequent conver- } \\
\text { sions, single end }\end{array}$ & 1.5 & 13 \\
\hline
\end{tabular}

The ADC conversion timing diagram is shown in Figure 4.1. Table 4.2 shows ADC register definitions and functions. The Application Programming Interface (API) for the ADC is listed in Appendix E.

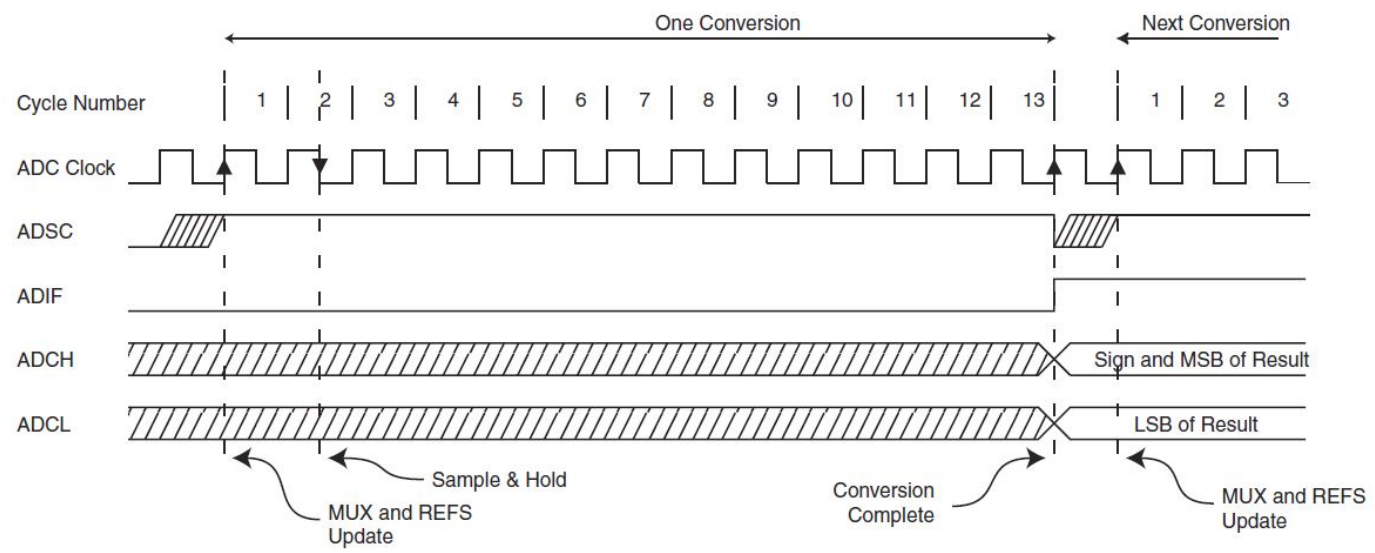

Figure 4.1: Analog to Digital Converter Timing Diagram for Single Conversion Mode 
Table 4.2: ADC Configuration

\begin{tabular}{|l||l|l|l|}
\hline $\begin{array}{l}\text { ADC } \\
\text { Parameter }\end{array}$ & $\begin{array}{l}\text { Microcontroller } \\
\text { Register and Bit(s) }\end{array}$ & Value & Value Interpretation \\
\hline \hline Reference Value & ADMUX(REFS1:0) & b01 & $\begin{array}{l}\text { AVCC with external ca- } \\
\text { pacitor at AREF pin, 5V }\end{array}$ \\
\hline ADC Clock & ADCSRA(ADPS2:0) & b010 & XTAL/4 \\
\hline ADC Trigger & ADCSRA(ADATE) & b0 & ADC auto trigger disable \\
\hline ADC Input Pin & $\begin{array}{l}\text { ADCSRB(MUX5) } \\
\text { ADMUX(MUX4:0) }\end{array}$ & b000000 & ADC0 \\
\hline $\begin{array}{l}\text { ADV value justi- } \\
\text { fication }\end{array}$ & ADMUX(ADLAR) & b1 & ADC left adjust result \\
\hline ADC Interrupt & ADCSRA(ADIE) & b0 & ADC interrupt disables \\
\hline
\end{tabular}

\subsection{Interrupt}

An external microcontroller interrupt triggers the Interrupt Service Routine (ISR) (see Chapter 8). The interrupt configuration for the microcontroller in shown in Table 4.3.

Table 4.3: Interrupt Configuration

\begin{tabular}{|l||l|l|l|}
\hline $\begin{array}{l}\text { Interrupt } \\
\text { Parameter }\end{array}$ & $\begin{array}{l}\text { Microcontroller } \\
\text { Register and Bit(s) }\end{array}$ & Value & Value Interpretation \\
\hline $\begin{array}{l}\text { Active Interrupt } \\
\text { pin }\end{array}$ & EIMSK(INT0) & b1 & Enable INT0 \\
\hline $\begin{array}{l}\text { Interrupt trigger } \\
\text { edge }\end{array}$ & EICRA(ISC10:00) & b011 & Rising edge triggered \\
\hline
\end{tabular}




\subsection{DAC}

The LTC2565 is an Octal 12-bit rail-to-rail DAC. The DAC block diagram is shown in Figure I.4 (Appendix I). Key features include:

- 8 individually configurable DAC units

- Synchronous or asynchronous update on each DAC

- Precision 10ppm $/{ }^{\circ} \mathrm{C}$ Max Deviation from Reference Voltage

- Selectable Internal or External Reference

- Power-On-Reset to Zero-Scale $(0 \mathrm{~V}) /$ Mid-scale (half of reference voltage)

In the internal feedback loop, one DAC output channel is set to the comparator reference input. The other four output channels are used to set the four phase shifter's control voltages, which are further described in Section 5.1. The DAC has a resolution defined in Equation 4.3

$$
D A C_{r e s l}=\frac{2 * V_{\text {ref }}}{2^{\text {bits }}}=\frac{2 \times 2.5}{2^{12}}=1.2 \mathrm{mV} / \text { quanta }
$$

The comparator has an internal error of $6 \mathrm{mV}$, or 5 quanta. The RF Power Detector's output slope is $37.3 \mathrm{mV} / \mathrm{dBm}$, thus the $\mathrm{DAC}$ resolution with respect to power is provided by Equation 4.4:

$$
\frac{37.3 m V}{1 d B} \times \frac{1 \text { quanta }}{1.2 m V} \approx 31 \text { quanta } / d B
$$

The DAC uses an SPI bus to communicate with the microcontroller. The LTC2656 
can operate with clock speeds up to $50 \mathrm{MHz}$; however, the DAC operates at a clock speed of $4 \mathrm{MHz}$. The maximum clock frequency for the SPI is one quarter the microcontroller's clock frequency, $\frac{f_{o s c}}{4}$. The microcontroller's operating frequency is $16 \mathrm{MHz}$.

The load sequence timing diagram is shown in Figure 4.2. The data word is 16-bits in length since the DAC is available in both 12 and 16 bits. The system only uses a 12 bit DAC: thus, the four Least Significant Bits (LSB) are not used.

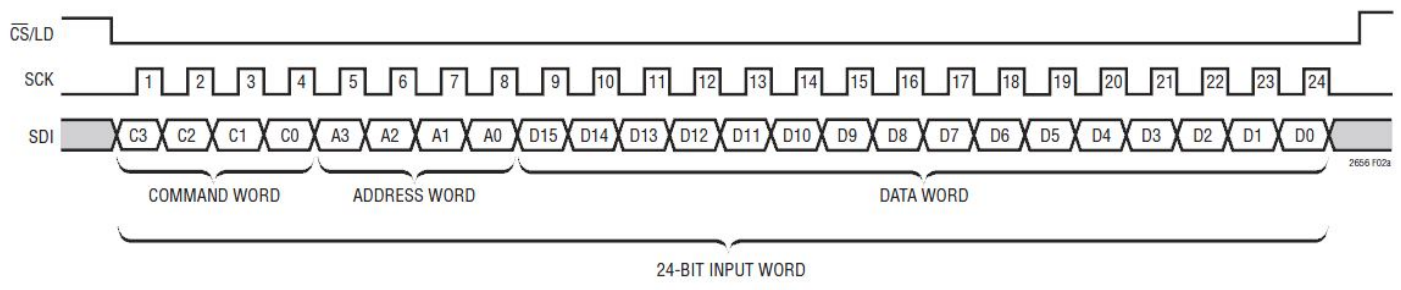

\section{Figure 4.2: LTC2656 DAC Load Sequence}

The DAC has a 24-bit input word, but the ATmega2560 is an 8-bit microcontroller (Figure 4.2). Each time a load sequence is sent to the DAC, the SPI Data Register (SPDR) is loaded sequentially three times. Tables 4.4 and 4.5 show the DAC command and address codes, respectively. For DAC and ADC API, refer to files: SPI_API.h, SPI_API.c and DAC_SPI.h in Appendix E. 
Table 4.4: DAC Command Codes

\begin{tabular}{|c|c|c|c|c|}
\hline \multicolumn{4}{|c|}{ Command } & \multirow[t]{2}{*}{ Operation } \\
\hline C3 & $\mathrm{C} 2$ & $\mathrm{C} 1$ & $\mathrm{Co}$ & \\
\hline 0 & 0 & 0 & 0 & Write to Input Register $n$ \\
\hline 0 & 0 & 0 & 1 & Update (Power Up) DAC Register n \\
\hline 0 & 0 & 1 & 0 & Write to Input Register n, Update (Power Up) All \\
\hline 0 & 0 & 1 & 1 & Write to and Update (Power Up) n \\
\hline 0 & 1 & 0 & 0 & Power Down $\mathrm{n}$ \\
\hline 0 & 1 & 0 & 1 & Power Down Chip (All DACs and Reference) \\
\hline 0 & 1 & 1 & 0 & Select Internal Reference (Power-Up Reference) \\
\hline 0 & 1 & 1 & 1 & Select External Reference (Power-Down Reference) \\
\hline 1 & 1 & 1 & 1 & No Operation \\
\hline
\end{tabular}

Table 4.5: DAC Address Codes

\begin{tabular}{|c|c|c|c|c|}
\hline \multicolumn{4}{|c|}{ Address 'n' } & Operation \\
\hline A3 & A2 & A1 & A0 & \\
\hline 0 & 0 & 0 & 0 & DAC A \\
\hline 0 & 0 & 0 & 1 & DAC B \\
\hline 0 & 0 & 1 & 0 & DAC C \\
\hline 0 & 0 & 1 & 1 & DAC D \\
\hline 0 & 1 & 0 & 0 & DAC E \\
\hline 0 & 1 & 0 & 1 & DAC F \\
\hline 0 & 1 & 1 & 0 & DAC G \\
\hline 0 & 1 & 1 & 1 & DAC H \\
\hline 1 & 1 & 1 & 1 & All DAC's \\
\hline
\end{tabular}




\subsection{Comparator}

A high-precision comparator enables voltage comparison between two signals with

difference in $\mathrm{mV}$ range; the voltages used in the system are $\approx 20 \mathrm{mV}$ apart. The LM339 is a Low Power Low Offset Voltage Quad Comparator. Key features include:

- Supply voltage 2 to $36 V_{D C}$

- Low input biasing current: 25nA

- Low supply current drain (0.8mA) independent of supply voltage

- Output voltage compatible with TTL, DTL, ECL, MOS and CMOS logic systems

- Response time of $1.3 \mu \mathrm{s}$

The simplified schematic of a single LM339 comparator is shown in Figure 4.3. The open collector comparator output requires a pull-up resistor to the $5 \mathrm{~V}$ rail; a $3.3 k \Omega$ resistor is used. 


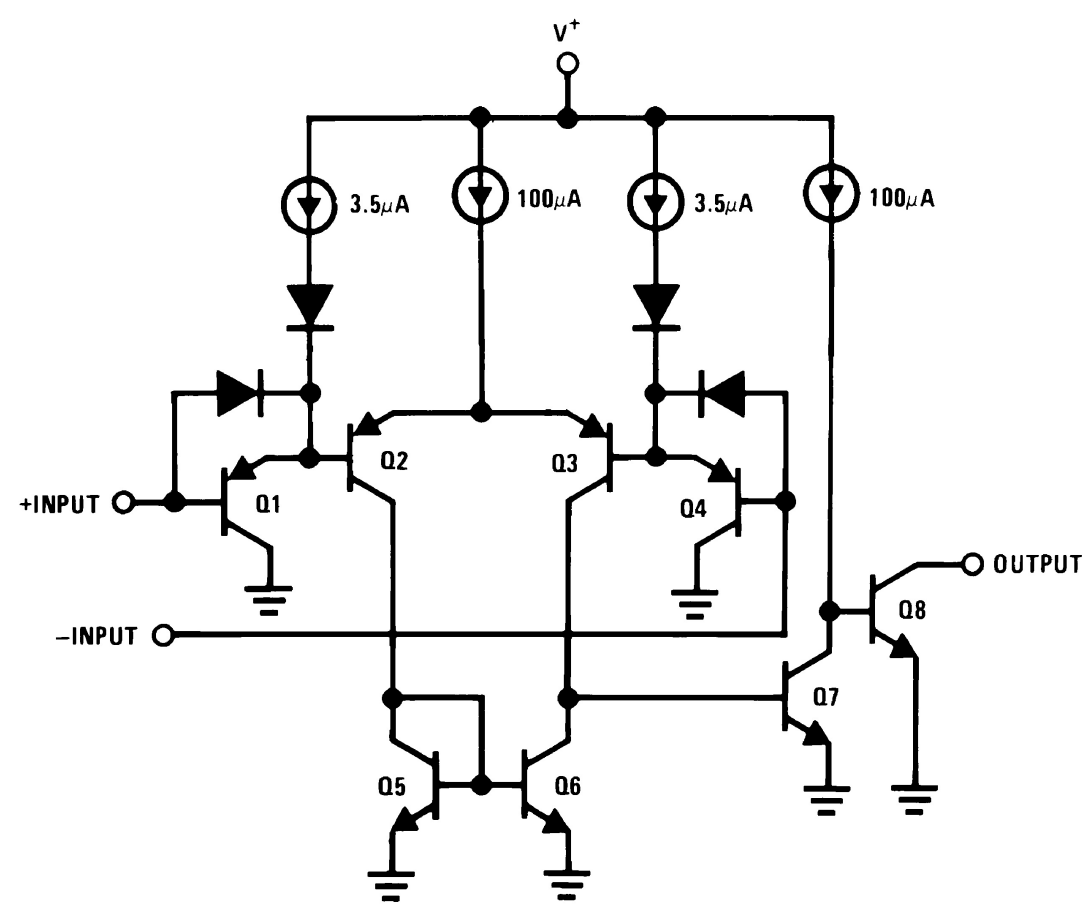

Figure 4.3: LM339 Schematic Diagram

A $0.1 \mu \mathrm{F}$ capacitor is used at the comparator output to remove high frequency glitching caused when inputs are within $20 \mathrm{mV}$ of each other. Although a capacitor delays the comparator output rise time, it is required to avoid output glitching. The comparator output triggers the microcontroller interrupt. A context switch is the process of storing and restoring the state (context) of a process so that execution can be resumed from the same point at a later time. If an interrupt triggers multiple times within a single context switch, the microcontroller keeps track of the trigger counts, and causes the microcontroller to go in multiple nested context switches. These multiple context switching cause the system to become unstable. The system unsuitability is due to the unknown and unstable state of the system variables. If the microcontroller enters an unstable state, a power cycle is executed. 


\section{CHAPTER 5}

\section{RF SYSTEM INTEGRATION}

This chapter describes the microcontroller control lines for the RF system. A 16-pin IDC interfaces the RF board to the digital board. The connector pinout (Table 2.1) and control lines are described in the following subsections.

\subsection{Phase Shifters}

Phase shifters control the four-patch inter-element phase shift to steer the radiation beam. The JSPHS-2484+ (MiniCircuits) is a $50 \Omega$ matched, $0^{\circ}$ to $180^{\circ}$ voltage-controlled phase shifter operating in the frequency range $2150 \mathrm{MHz}$ to $2484 \mathrm{MHz}$. The datasheet provides voltage-to-phase data at $2150 \mathrm{MHz}, 2300 \mathrm{MHz}$ and $2484 \mathrm{MHz}$. Linear interpolation between $2300 \mathrm{MHz}$ and $2484 \mathrm{MHz}$ yields voltage-to-phase data at $2400 \mathrm{MHz}$ as shown in Figure 5.1. 


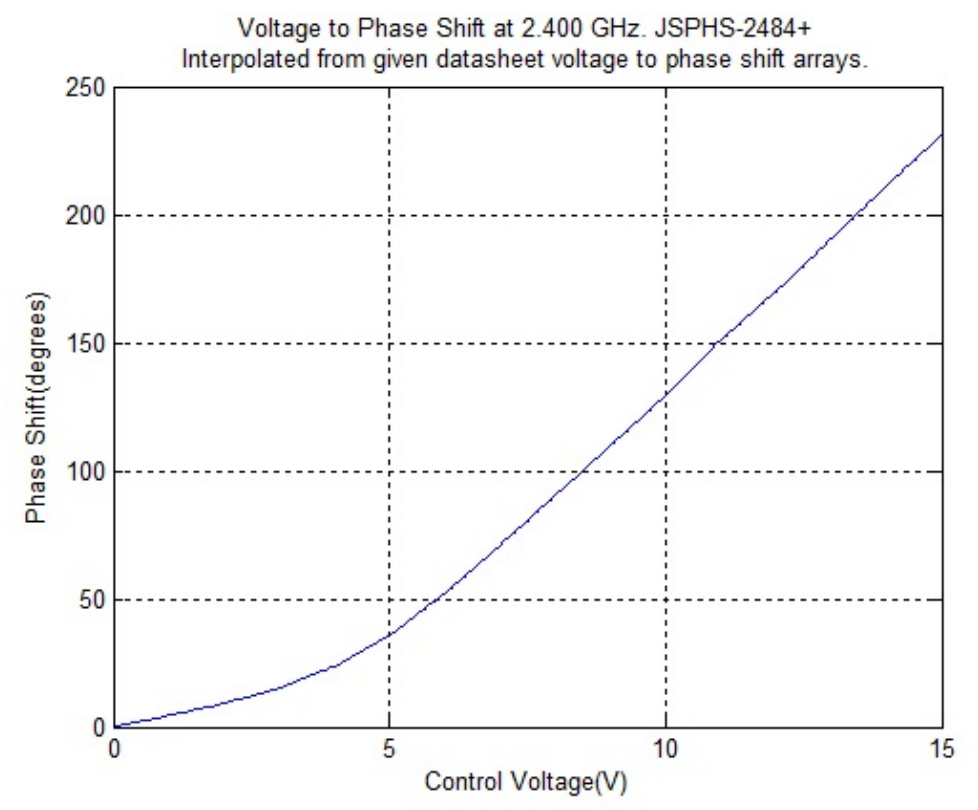

Figure 5.1: JSPHS-2484+ Phase Shifter, Interpolated Data

Individual phase shifters control the relative signal phase with respect to each path. The DAC was initially intended to set control voltages for the phase shifters directly. This requires DAC analog output voltages from 0V to $15 \mathrm{~V}$. DACs with $15 \mathrm{~V}$ output capability are limited, thus a 5V rail DAC was chosen. The LTC2656 DAC was selected to set phase shifter control voltages. An op-amp is required to increase the DAC output voltage range from $0-5 \mathrm{~V}$ to $0-15 \mathrm{~V}$; thus a gain of +3 is required. Figure 5.2 shows an op-amp in a non-inverting gain configuration. Equation 5.1 shows the input and output voltage relationship.

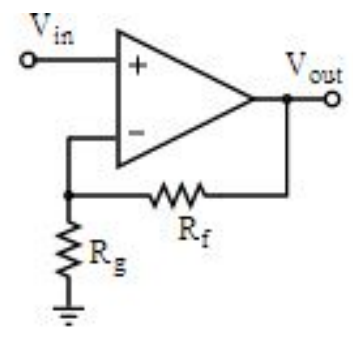

Figure 5.2: Operational Amplifier in Non-Inverting Configuration 


$$
V_{\text {out }}=V_{\text {in }}\left(1+\frac{R_{f}}{R_{g}}\right)
$$

For improved system performance a stable rail-to-rail op-amp was used. Each of the phase shifters require a voltage control line and an op-amp. To reduce component count, a quad pack op-amp (LT6005) was selected. Key Features of the LT6005 include:

- Wide supply range $1.6 \mathrm{~V}$ to $16.0 \mathrm{~V}$

- Low supply current, $1 \mu \mathrm{A}$

- Low input bias current, 90pA

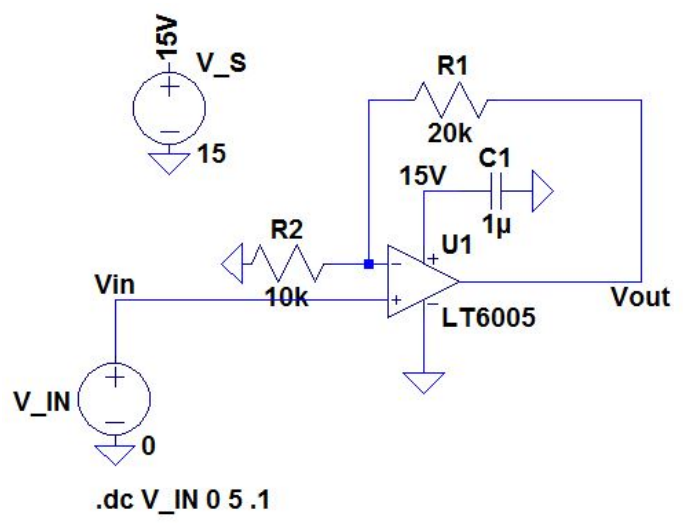

Figure 5.3: LTSpice Simulation Schematic, LT6005 Non-Inverting Gain Op-Amp Circuit

Figure 5.3 shows an LT6005 op-amp LTspice simulation. The simulation in Figure 5.3 assumes ideal resistors and capacitors. To model a practical circuit, a Monte Carlo Analysis was completed using variable resistor and capacitors values. Variations in resistor values cause control voltage deviations and phase offset errors, which will affect the beam direction. 
Analyses with resistor tolerances ranging from $5 \%$ to $0.01 \%$ were completed. The $0.01 \%$ tolerance had the best performance; the most cost effective option utilizes $0.5 \%$ tolerance resistors. The Monte Carlo analysis schematic and results are displayed in Figure 5.4. The response is expanded to highlight the area of maximum error, which occurs when input voltages are close to the $5.0 \mathrm{~V}$ rail. Figure 5.5 shows results from $4.95 \mathrm{~V}$ to $5.00 \mathrm{~V}$. The measured output saturation voltage for the op-amp is $14.97 \mathrm{~V}$ for a $15.00 \mathrm{~V}$ rail. The extreme cases are:

- $V_{\text {out }}=14.97 \mathrm{~V}$ at $V_{\text {in }}=4.95 \mathrm{~V}$

- $V_{\text {in }}=5.00 \mathrm{~V}$ outputs $V_{\text {out }}=14.92 \mathrm{~V}$

This Monte Carlo Analysis was iterated 50 times, which constitutes $85 \%$ of the Gaussian distribution at $0.5 \%$ tolerance. [10]

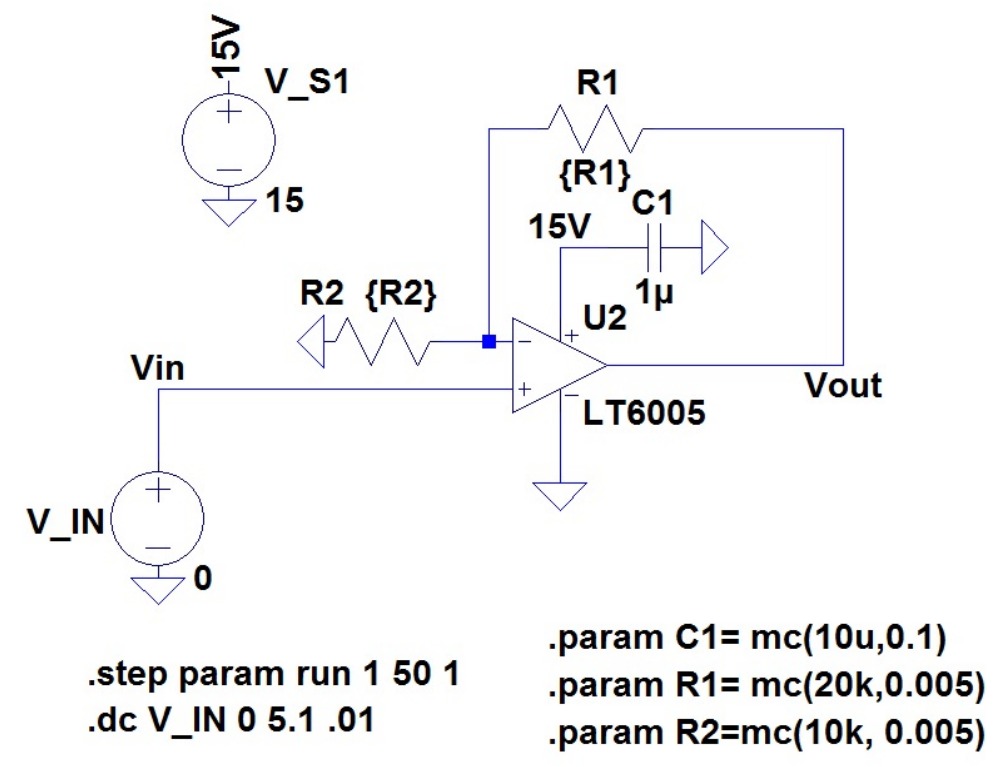

(a) Schematic

Figure 5.4: LTSpice Simulation results, LT6005 Op-amp Non-Inverting Gain Circuit 


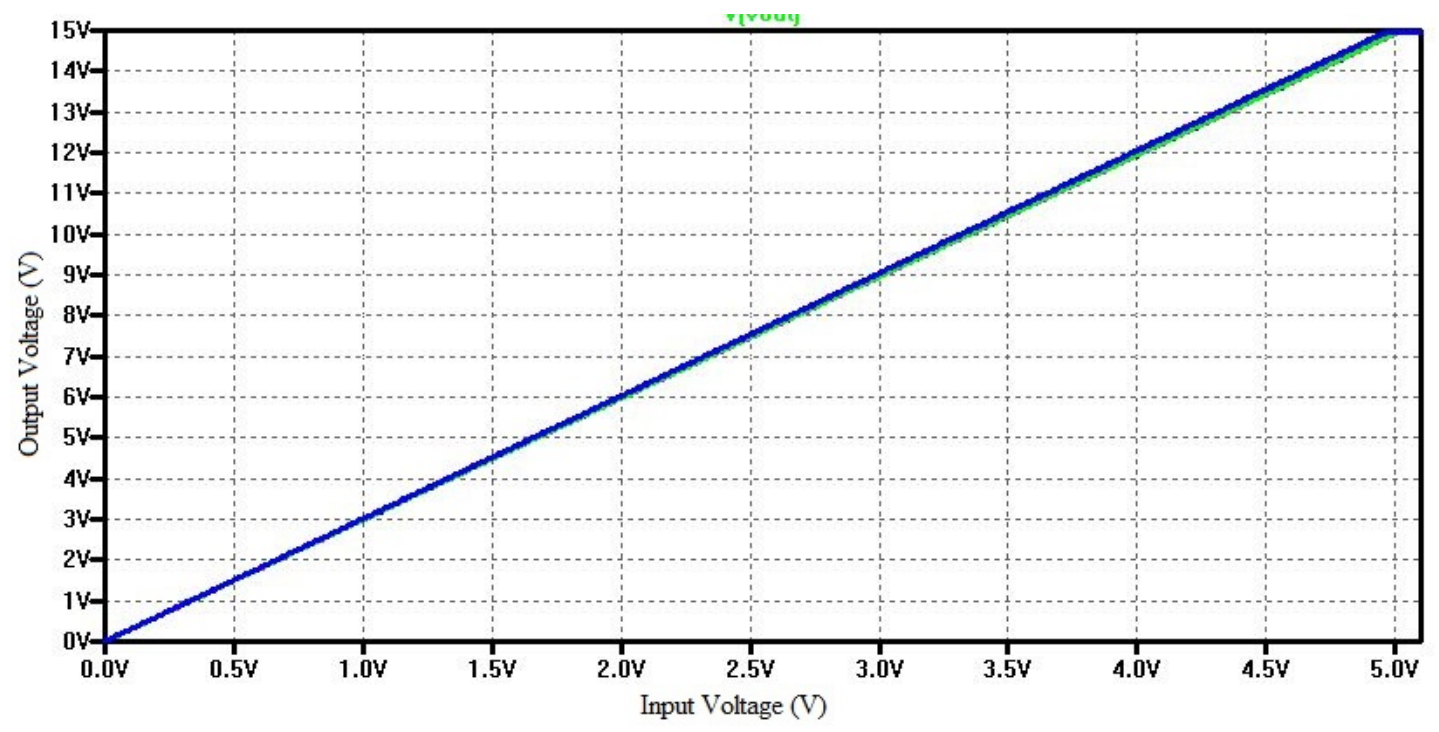

(b) Result

Figure 5.4: LTSpice Simulation results, LT6005 Op-amp Non-Inverting Gain Circuit

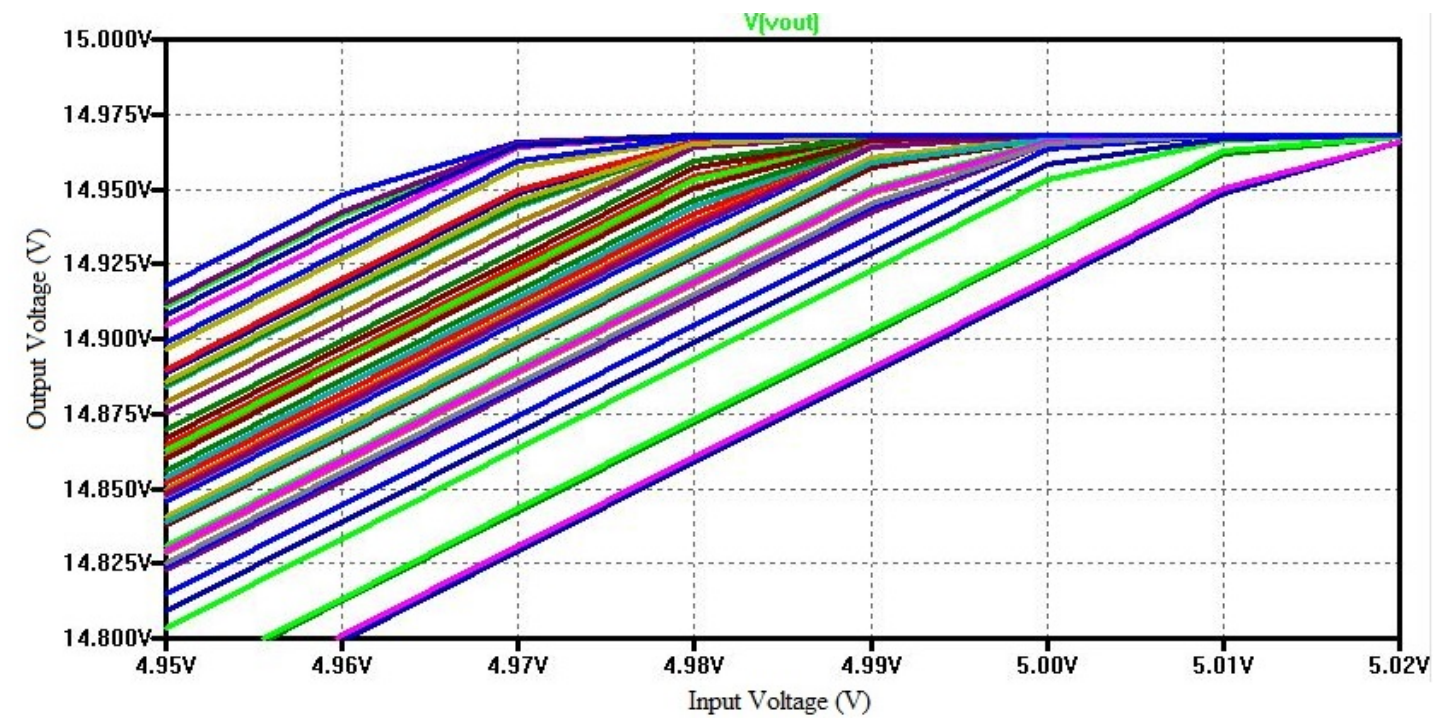

Figure 5.5: Monte Carlo Analysis for Amplification Circuit, Expanded View 


\subsection{RF Single Pole Double Throw (SPDT) Switch}

SPDT switches determine the Antenna and RF system modes: transmit or receive. Two control lines control the SPDT switch position. The AS179-92LF is a $20 \mathrm{MHz}$ to $3.0 \mathrm{GHz}$ GaAs SPDT switch. The AS179-92LF's pinout and block diagram is shown are Figure 5.6.

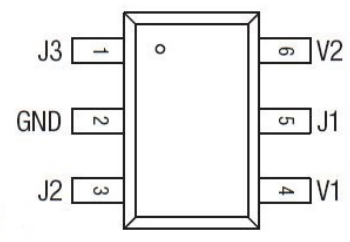

(a) Pinout

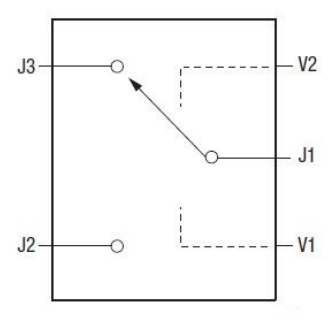

(b) Block Diagram

\section{Figure 5.6: AS179-92LF, SPDT, Pin-out and Block Diagram}

Table 5.1 shows the SPDT truth table. As defined in Figure 5.6b, V1 and V2 are control lines while $\mathrm{J} 1, \mathrm{~J} 2$, and $\mathrm{J} 3$ are RF lines. $\mathrm{J} 1$ is the common line while $\mathrm{J} 2$ and $\mathrm{J} 3$ are selectable. Table 5.2 shows the system truth table for the receive (RX) and transmit (TX) modes. For the transmit (TX) mode, J1 is connected to $\mathrm{J} 3$; for receive (RX) mode, $\mathrm{J} 1$ is connected to $\mathrm{J} 2$.

Table 5.1: Truth Table for SPDT, $V_{H I G H}=2 \mathrm{~V}$ to $5 \mathrm{~V}, V_{L O W}=0 \mathrm{~V}$ Insertion Loss $=0.5 \mathrm{~dB}$, and Isolation $=22 \mathrm{~dB}$

\begin{tabular}{|l|l||l|l|}
\hline $\mathrm{V} 1$ & $\mathrm{~V} 2$ & $\mathrm{~J} 1$ to J2 connection & $\mathrm{J} 1$ to J3 connection \\
\hline \hline$V_{H I G H}$ & 0 & Isolation & Insertion Loss \\
\hline 0 & $V_{H I G H}$ & Insertion Loss & Isolation \\
\hline
\end{tabular}


Table 5.2: SPDT, Antenna Mode Truth Table

\begin{tabular}{|l|l||l|}
\hline SPDT1(V1) & SPDT0(V2) & Mode \\
\hline \hline 1 & 0 & TX \\
\hline 0 & 1 & RX \\
\hline
\end{tabular}

\subsection{RF Single Pole Triple Throw (SP3T) Switch}

The SP3T switches determine the tetrahedral structure's active face. The HMC245QS16 is a SP3T RF switch is a GaAs-based monolithic microwave integrated circuit (MMIC) operating in the frequency range DC to $3.5 \mathrm{GHz}$. The SP3T switch's pinout and functional diagram is shown in Figure 5.7.

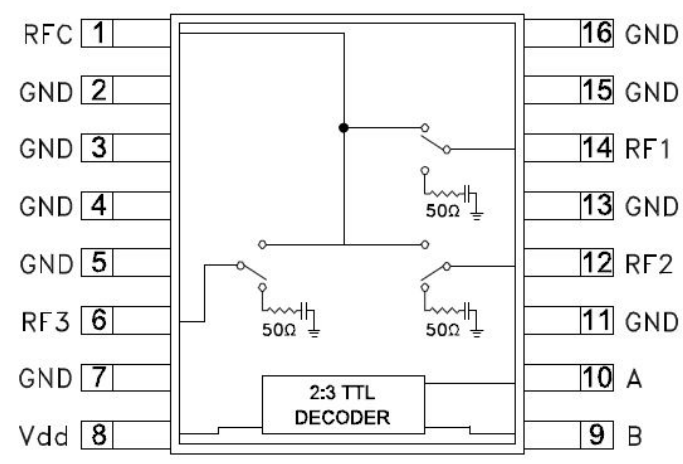

Figure 5.7: HMC245QS16 SP3T RF Switch Pin-out and Functional Diagram

Table 5.3 shows a truth table for the HMC245QS16 SP3T RF switch. Control lines A and B are compatible with both TTL (Transistor-Transistor Logic) and CMOS (Complementary Metal-Oxide Semiconductor) logic levels. Voltage ranges are defined as 0 to $0.8 \mathrm{~V}$ for $L O W$ and $2.0 \mathrm{~V}$ to $5.0 \mathrm{~V}$ for $H I G H$. The MMIC exhibits an insertion loss of $0.6 \mathrm{~dB}$ and isolation of $35 \mathrm{~dB}$ between $\mathrm{RF}$ ports at $2.4 \mathrm{GHz}$. 
Table 5.3: Truth Table for HMC245QS16 and the Active Antenna Face

\begin{tabular}{|c|c||c|c|}
\hline \multicolumn{2}{|c||}{ Control Input } & \multicolumn{2}{c|}{ Signal Path State } \\
\hline A & B & RF COM to: & Active Face \\
\hline \hline Low & Low & RF1 & 1 \\
\hline High & Low & RF2 & 2 \\
\hline Low & High & RF3 & 3 \\
\hline High & High & All Off & All Off \\
\hline
\end{tabular}

\subsection{Voltage Control Oscillator (VCO) Control Line}

The JTOS-2700V is a linearly tunable voltage controlled oscillator (VCO) for the frequency range 2050 to $2700 \mathrm{MHz}$. VCO Frequency Tuning and output power data are shown in Figure 5.8. 
JTOS-2700V

Frequency and Tuning Sensitivity $\left(\right.$ at $\left.+25^{\circ} \mathrm{C}\right)$

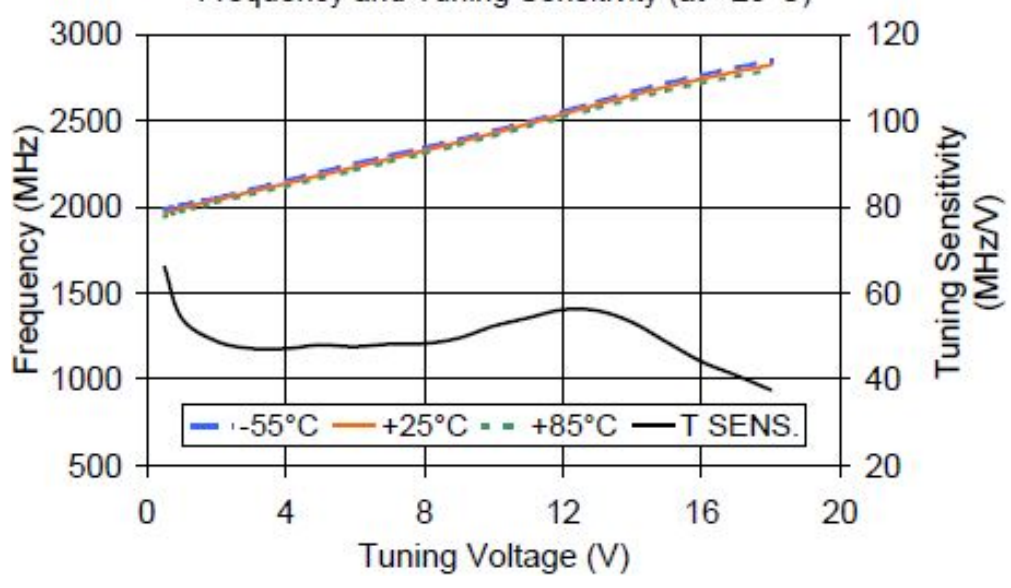

(a) Frequency Tuning JTOS-2700V

Power Output

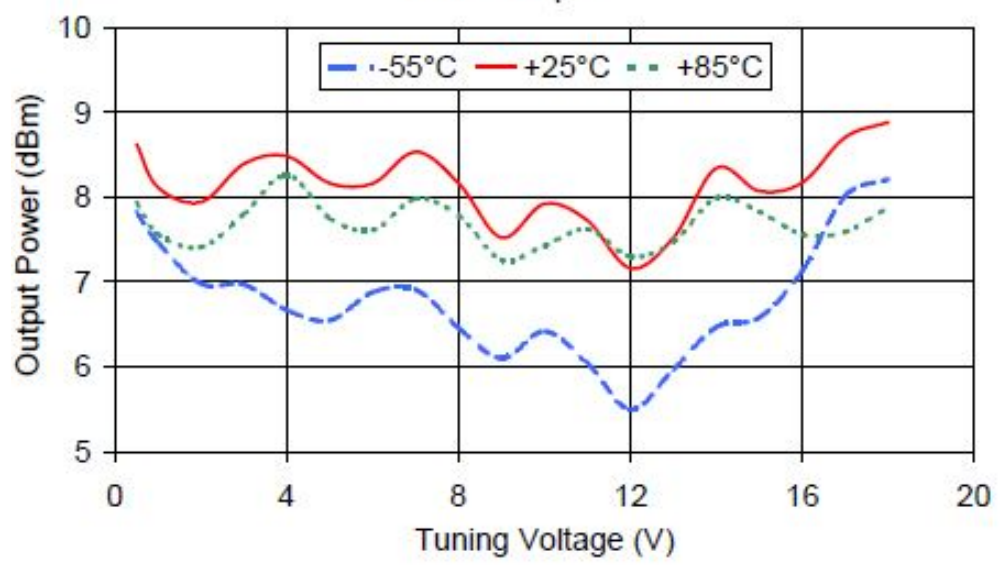

(b) Output Power

\section{Figure 5.8: JTOS-2700V, VCO, datasheet characteristic}

Figure $5.8 \mathrm{a}$ shows that a control voltage of $9.18 \mathrm{~V}$ is required to produce a $2.4 \mathrm{GHz}$ signal, the RF system's operating frequency. The antenna resonance frequency has an engineering tolerance of $5 \%$ at $2.4 \mathrm{GHz}$ [1], which requires a tunable VCO control line. Figure 5.9 shows the VCO tuning circuit with output voltages from $7.5 \mathrm{~V}$ to $10 \mathrm{~V}$. Table 5.4 shows output voltages when the tunable potentiometer is in the range $1 \Omega$ to $5 k \Omega$. A 14 -turn tunable $5 k \Omega$ potentiome- 
ter provides fine adjustment for the VCO output frequency. High tolerance 1\% resistors are used for $R 1$ through $R 4$.

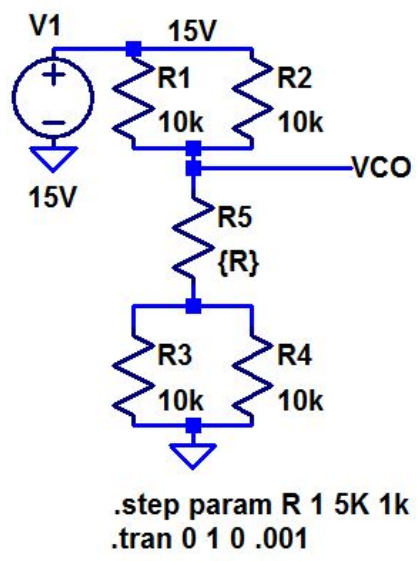

Figure 5.9: VCO tuning Schematic, R5 is the tunable resistor

Table 5.4: VCO Tuning Circuit Result

\begin{tabular}{|c|c|}
\hline Potentiometer resistance $(\Omega)$ & VCO control voltage $(\mathrm{V})$ \\
\hline 1 & 7.50 \\
\hline $1 \mathrm{k}$ & 8.18 \\
\hline $2 \mathrm{k}$ & 8.75 \\
\hline $3 \mathrm{k}$ & 9.23 \\
\hline $4 \mathrm{k}$ & 9.64 \\
\hline $5 \mathrm{k}$ & 10 \\
\hline
\end{tabular}




\section{CHAPTER 6}

\section{PRINTED CIRCUIT BOARD DESIGN}

CadSoft EAGLE PCB Design Software was used to design the printed circuit

board (PCB) schematic and layout. PCB schematic and layout can be found in Appendices F and G, respectively.

\subsection{Schematic}

The schematic was created to add the maximum testability in the PCB. The following are some of the key testability features added to the PCB design:

- Zero ohm resistors to isolate power supplies and ICs

- Zero ohm resistors to isolate outputs and inputs for testability

- DIP switch and header pins to set SPDT and SP3T control signal manually and LEDs to show the status

- Test points at most microcontroller input/outputs (I/Os)

- LED Display Driver(LM3914) to show the received power RF power detector.

- LEDs to check the power statuses

- On broad ISP programmer pinout 
The ICs used required $5 \mathrm{~V}$ and $15 \mathrm{~V}$ power rails. To avoid the need for two power supplies, a 20V AC to DC wall transformer was used with power regulators. A barrel connector was used to connect the $20 \mathrm{~V}$ power supply to the board. ON Semi's NCP1117ST50T3G was used to achieve 5V rail and Texas Instrument part LM3480 was used to achieve $15 V$ rail. Linear Technology LT1761 was used to set the $2.5 \mathrm{~V}$ DAC reference voltage.

\subsection{Layout}

A two-layer PCB was chosen for the layout. A full copper pour on the top and bottom layers was used as the ground. The advantage of large group plane is its low inductance and resistance. Routing the signals and power lines in the group pour helps maintain a good signal value by creating capacitance between the signal or power and the ground signal. A section of copper pour was cut out from the full copper pour to act as analog ground. The full ground plane and analog ground plane were connected via $22 \mathrm{nH}$ inductor, which prevents the high frequency switching ground plane currents from affecting the analog voltages. The analog plane was used under the DAC and the Amplifier. The layout was designed so that traces would have a minimal number of vias. 


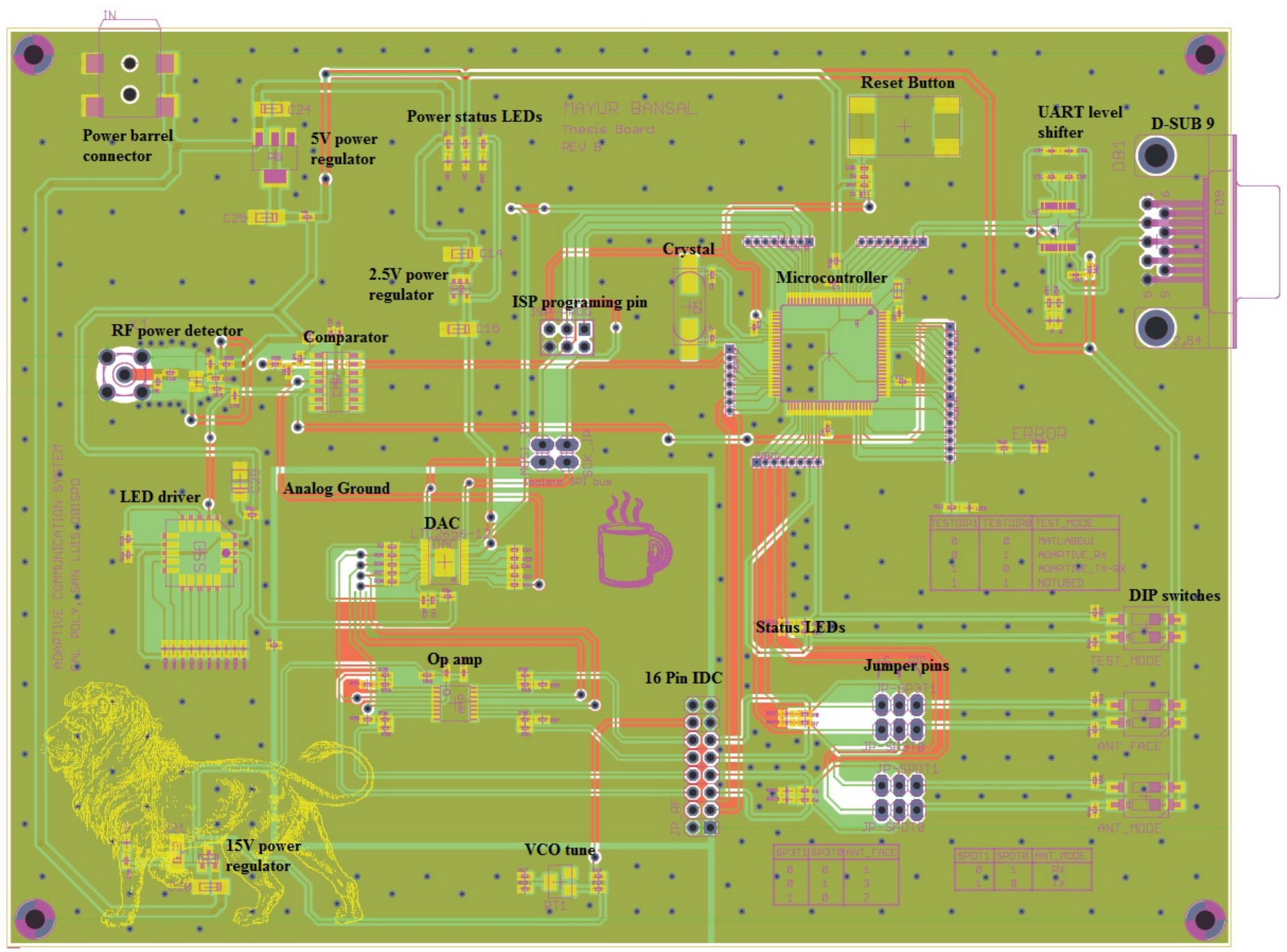

Figure 6.1: PCB layout

Figure 6.1 shows the layout of the subsequent PCB. All the main components are marked in the Figure with accompanying text. All connectors were placed so that they are as close to their mates as possible. 


\section{CHAPTER 7}

\section{GRAPHICAL USER INTERFACE (GUI) CONTROL SYSTEM}

The MATLAB GUI is designed to test system functionality. The GUI configures all system variables for beam steering and can be set to transmit or receive mode. MATLAB uses $R S$-232, a serial protocol, to communicate between the computer and control board. A 9-pin D-subminiature (D-sub) connector is used for RS-232 communication; the pinout is shown in Figure 7.1 .

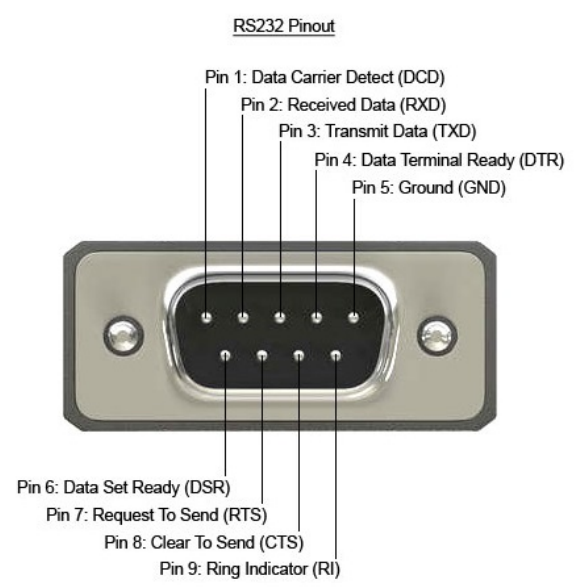

Figure 7.1: RS-232, 9-pin D-sub connector pinout

MATLAB has a built-in serial communication library. Data is sent from the MATLAB GUI to the microcontroller via a one-way data link. The microcontroller interprets the RS-232 data and sets the system variables accordingly. The GUI screen is shown in Figure 7.2. 


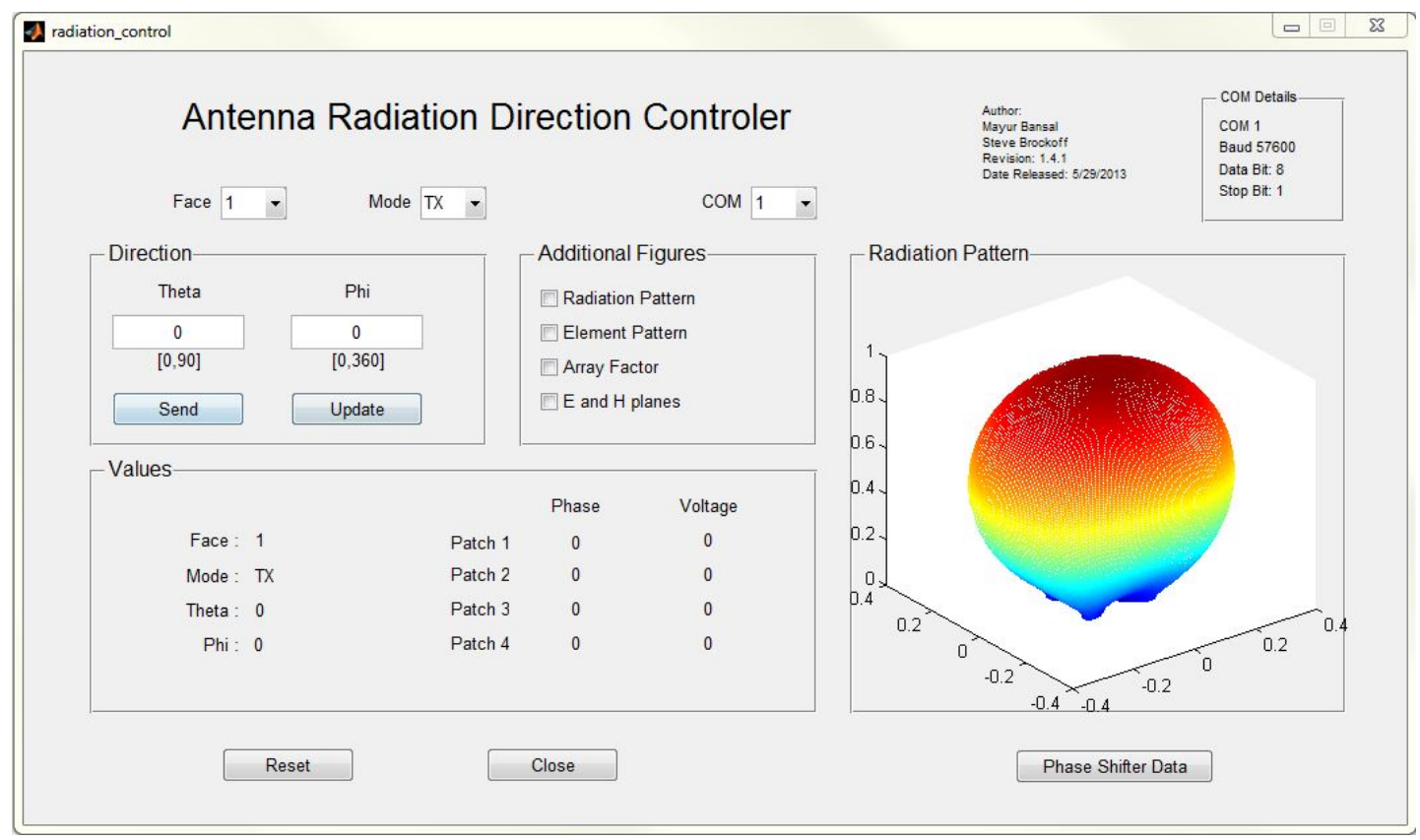

Figure 7.2: MATLAB GUI main screen

\subsection{GUI Inputs and Features}

MATLAB GUI has four user interface fields.

1. Drop down menus: Face, Mode and COM

2. Edit Text: Theta and Phi

3. Push Buttons: Reset, Close, Phase Shifter Data, Send and Update

4. Checkbox: Radiation Pattern, Element Pattern, Array Factor and E and H Planes

\subsubsection{Drop Down Menus}

The three drop down menus are Face, Mode and COM port. The Face drop-down menu has three options: 1, 2, or 3. Figure 7.3 shows the GUI screen-shot with 
user options. The selection controls the adaptive antenna face. The antenna face is controlled using SP3T switches. The antenna faces 1, 2, and 3 are labeled on the tetrahedral antenna structure.

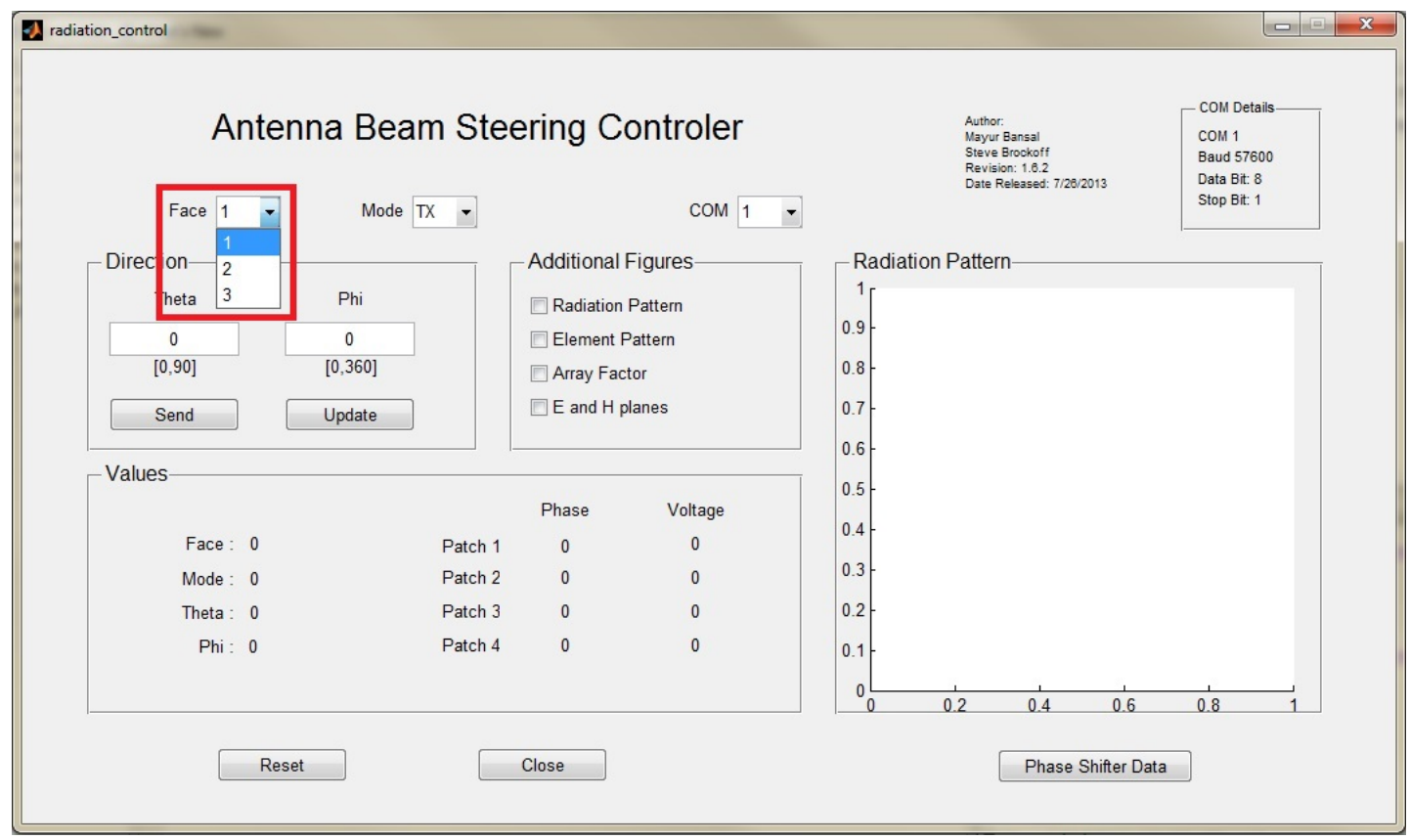

\section{Figure 7.3: MATLAB GUI screen with Face drop down menu}

The Mode drop down menu has two options; TX (transmit) or RX (receive), which are controlled by SPDT switches. Figure 7.4 shows the GUI screen-shot with the Mode drop down menu open. 


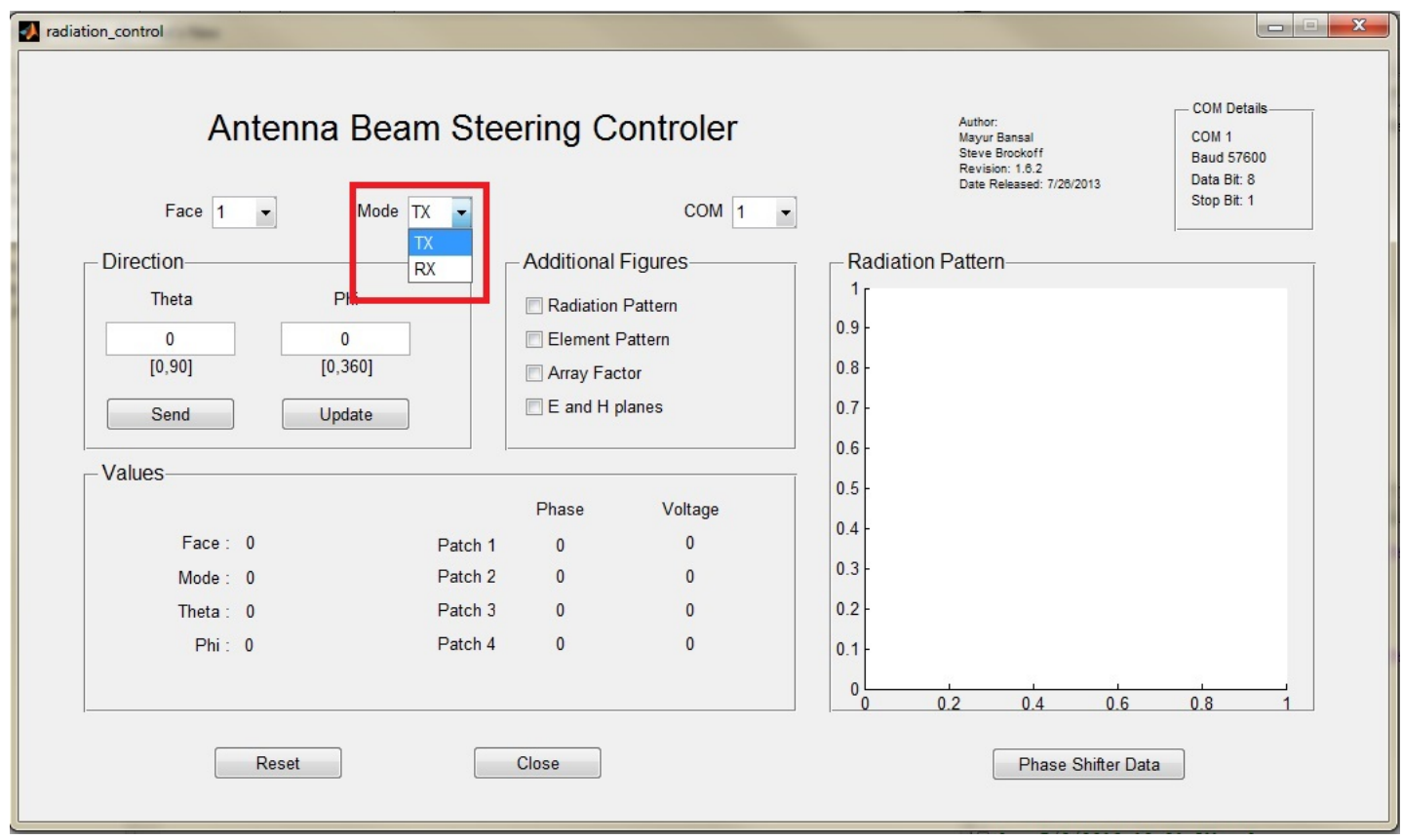

Figure 7.4: MATLAB GUI Screen with Mode Drop-Down Menu

RS-232 uses the COM port set by the COM drop down menu. A TRENDnet TU-S9 (Figure 7.5) USB to RS-232 converter cable connects the PC to the Control Board. On Microsoft Windows, the Device Manager shows the COM port that the converter cable is connected to on the computer. As shown by the red highlighted box in Figure 7.6.

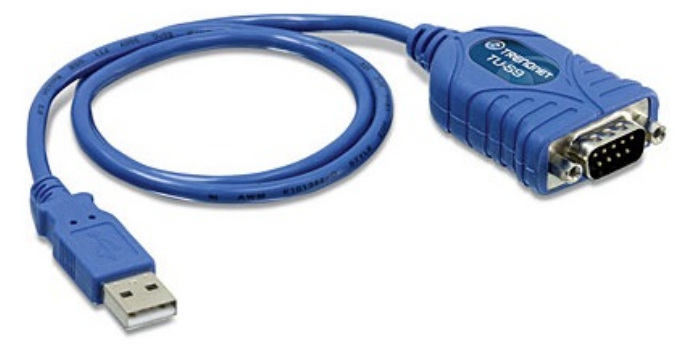

Figure 7.5: TRENDnet TU-S9, USB to RS-232 Converter Cable 


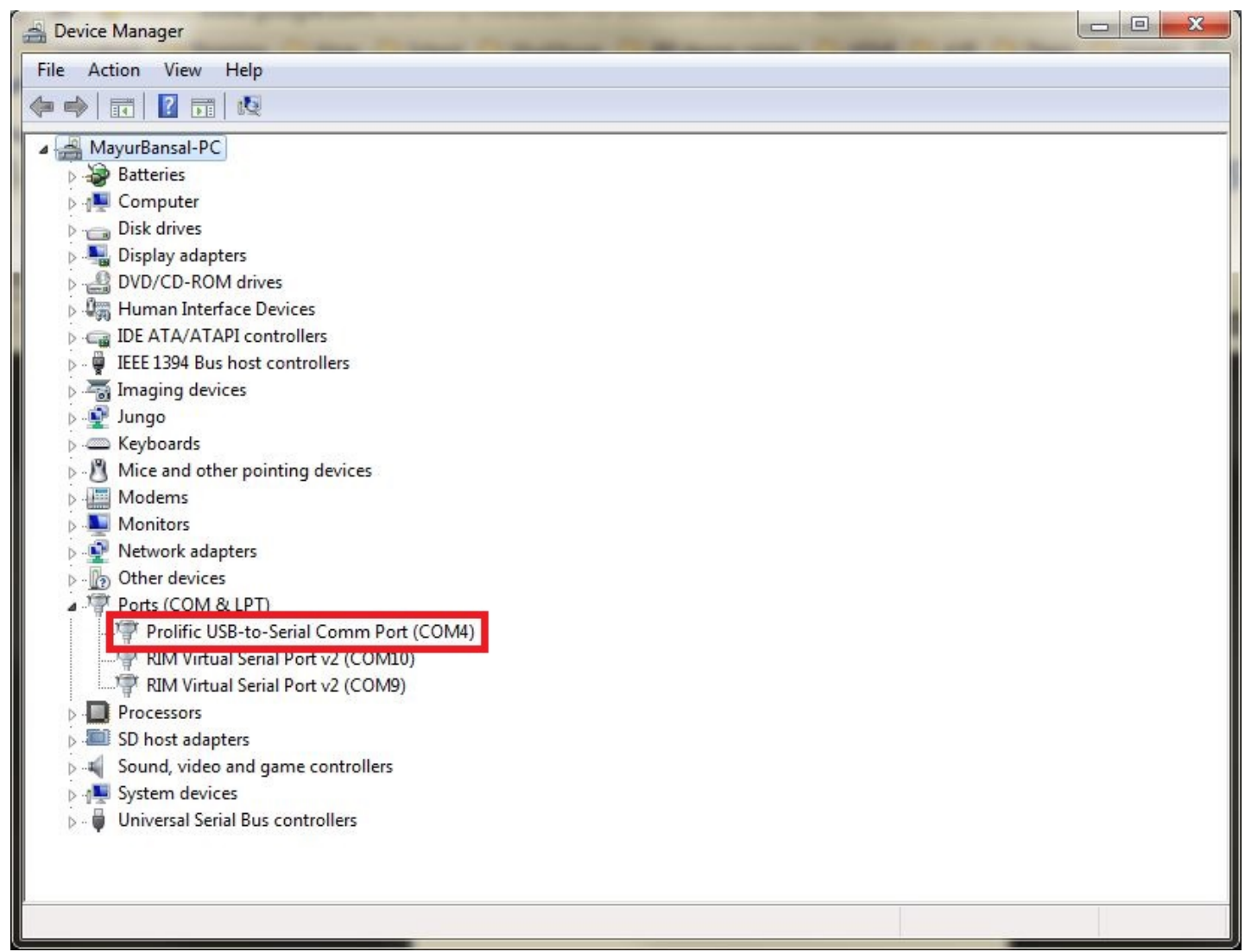

Figure 7.6: Device Manager screen shot with COM port highlighted

Once the COM port is known, the COM drop-down menu sets the COM port value. The blue highlighted box in Figure 7.7 shows the default range of COM port values, while the red highlighted box shows the serial connection details. The BAUD rate (pulses per second) of 57600, eight data bits, and one stop bit is used. 


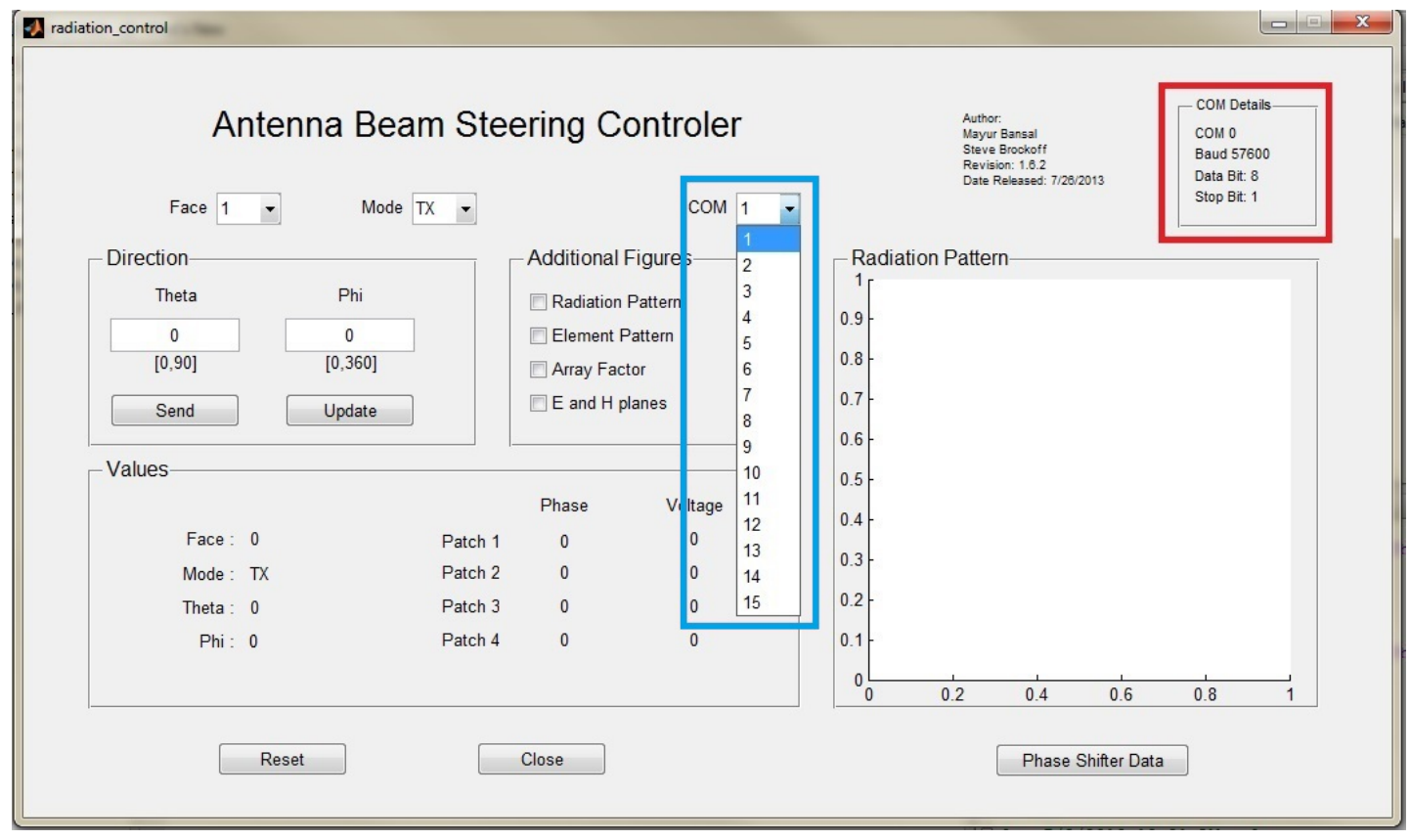

Figure 7.7: MATLAB GUI screen with COM drop down menu

\subsubsection{Edit Text}

Theta $(\theta)$ and Phi $(\phi)$ are two editable text fields. Figure 7.8 shows theta and phi in the spherical coordinate system. The limits on $\theta$ are $0^{\circ}$ to $90^{\circ}$ or 0 to $\frac{\pi}{2}$ radians because it is impractical to direct radiation below the antenna plane. The limits on $\phi$ are 0 to $360^{\circ}$ or 0 to $2 \pi$ radians. 


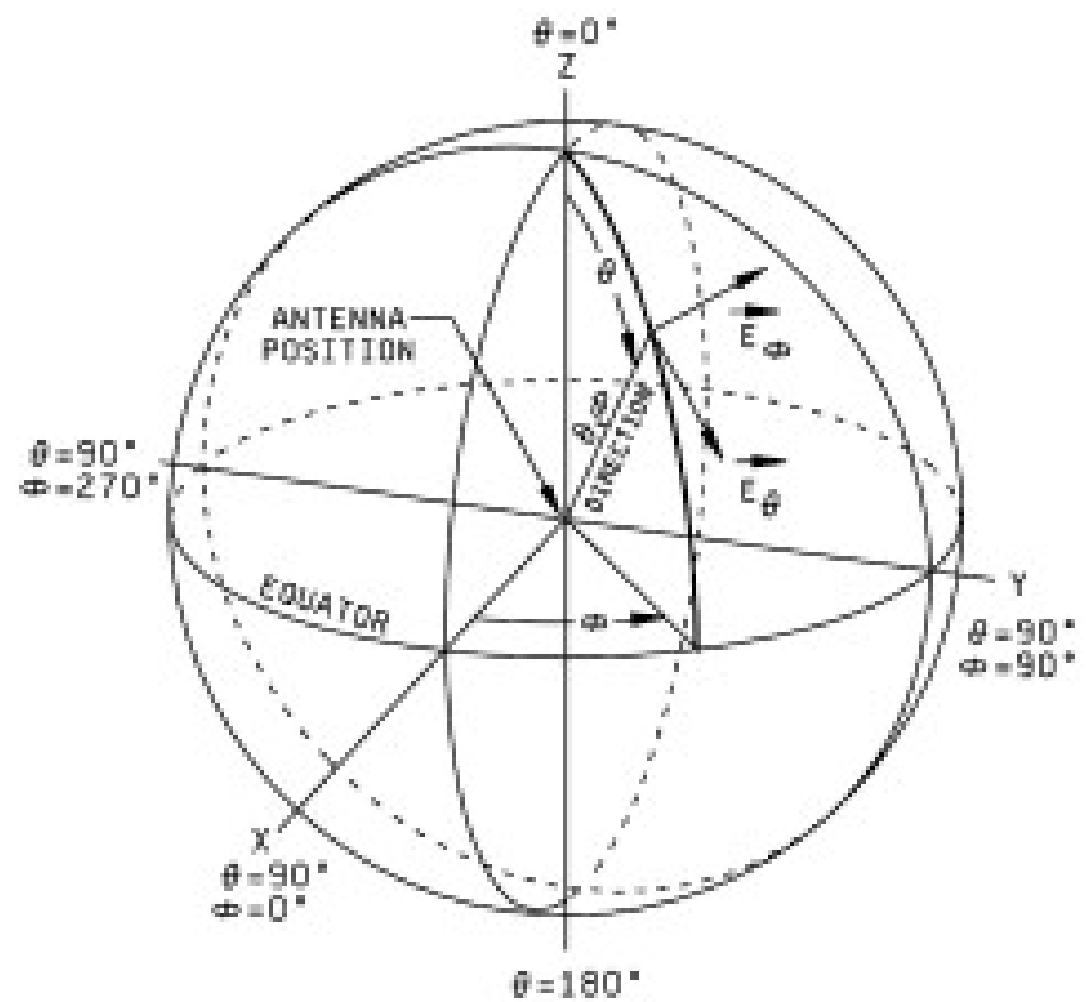

Figure 7.8: Antenna Radiation Pattern Coordinate system

$\theta$ and $\phi$ set the antenna beam direction, or its radiation pattern's main lobe. The calculated control angles and phase shifter voltages from the $\theta$ and $\phi$ fields are shown in the green highlighted box in Figure 7.9. The antenna radiation pattern is displayed in the GUI right panel, highlighted with a red box. The blue highlighted box shows the achievable main beam direction in $\theta$ and $\phi$ coordinates. The offset between theoretical and achievable is due to phase shifter limitations $[1]$. 


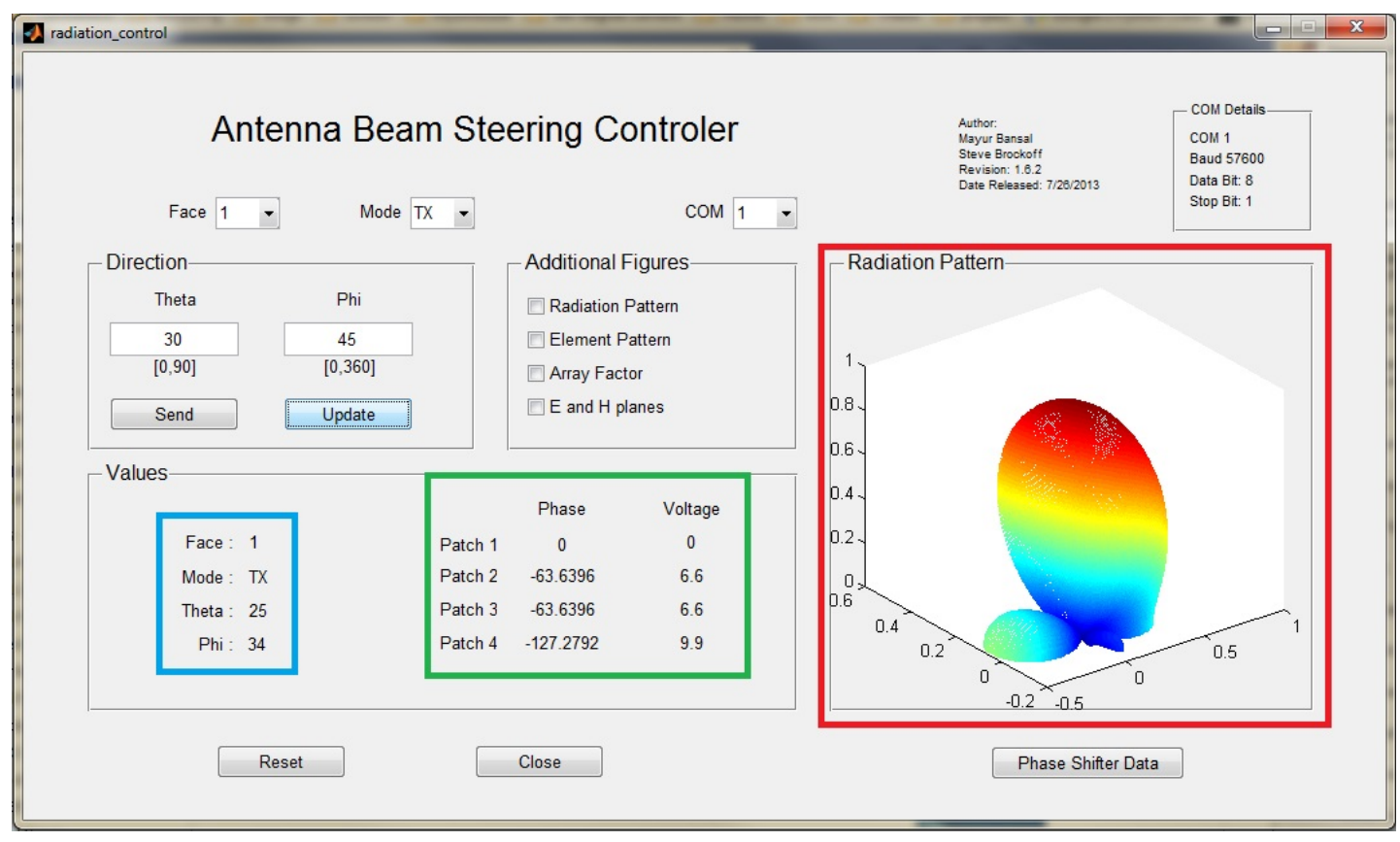

Figure 7.9: MATLAB GUI Screen Shot, $\theta$ and $\phi$ Control Variables

\subsubsection{Push Buttons}

There are five GUI push buttons: Reset, Close, Phase Shifter Data, Send, and Update. The Reset button returns the GUI to its default state. The Close button closes the MATLAB GUI, but maintains all open figures defined in Section 7.1.4. The Phase Shifter Data button shows the Phase Shifter JSPHS-2484+ characteristic data interpolated from the associated datasheet, which is shown in Figure 7.10. 


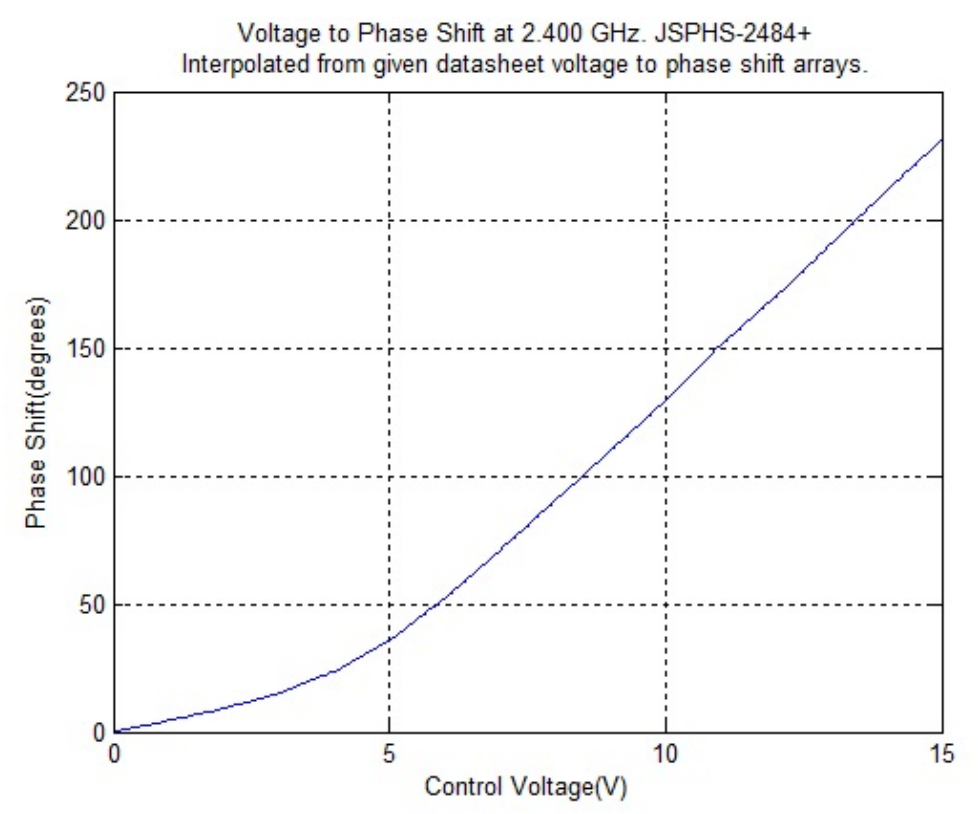

Figure 7.10: Phase Shifter JSPHS-2484+ Interpolated Data

The Update button initiates phase shifter control voltage and angle value calculations from theta and phi values. The Update button also refreshes the Value and Radiation Pattern panel, and creates the plots selected from the Additional Figures panel. In addition to Update button's function, theSend Button also transmits the data packet to the microcontroller. Transmit data packet structure is described in Section 7.2.

\subsubsection{Additional Figure Checkboxes}

The four checkboxes that create additional information plots for antenna radiation pattern are Radiation Pattern, Element Pattern, Array Factor and E and $H$ Planes. Figure 7.11 shows all the additional plots for $\theta=30^{\circ}$ and $\phi=45^{\circ}$. The $E$ and $H$ planes checkbox creates an addition plot for all previously selected options in the Additional Figures panel. 


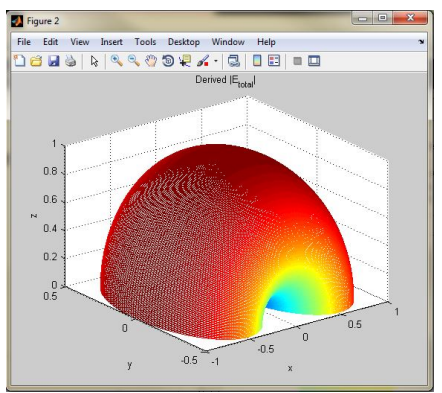

(a) Element Pattern

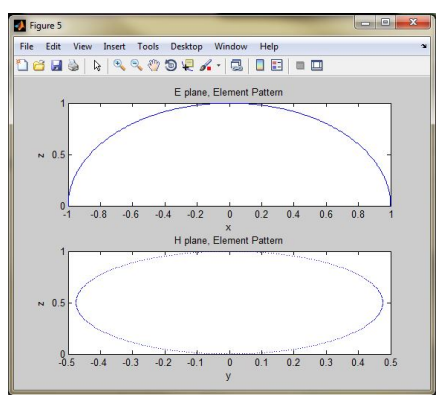

(d) E and H planes, Element Pattern

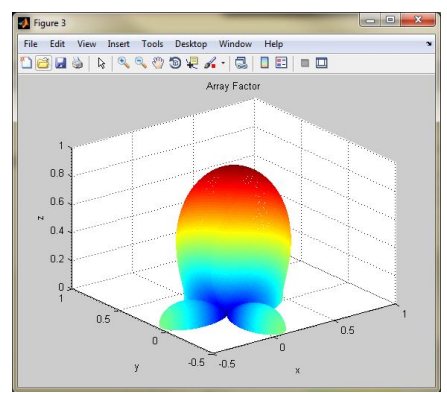

(b) Array Factor

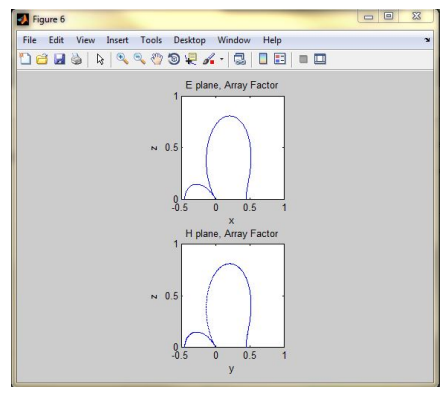

(e) E and H planes, Array

Factor

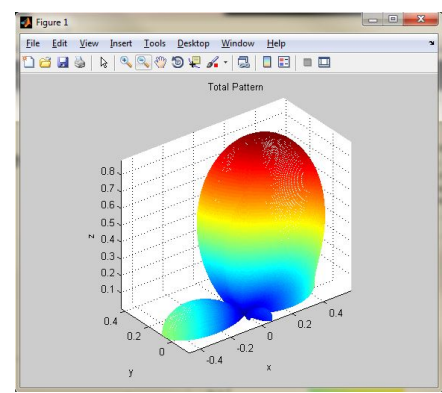

(c) Total Pattern

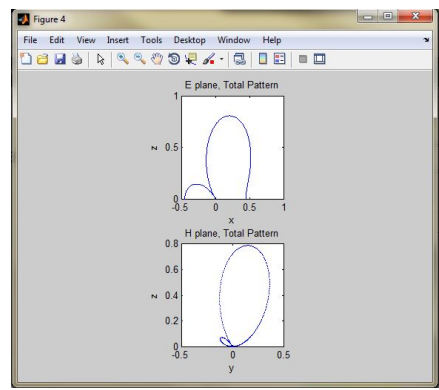

(f) E and H planes, Total Pattern

Figure 7.11: Checkbox Selected Images, with $\theta=30^{\circ}$ and $\phi=45^{\circ}$

\subsection{Communication Protocol}

Figure 7.12 shows a single RS-232 physical frame. The protocol consists of a start bit, eight data bits, and one stop bit. The start bit is usually HIGH. Data bits are in order of least significant bit (LSB) to most significant bit (MSB). The stop bit is usually LOW.

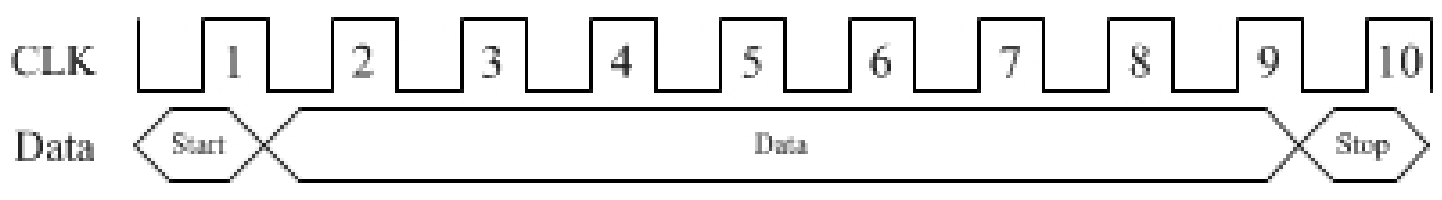

Figure 7.12: RS-232 Frame 
The protocol data packet for communication between the microcontroller and MATLAB GUI include mode value, face value and four phase shifter control voltages. Figure 7.13 shows the timing diagram for a data packet. RS-232 data bits are transmitted as an 8-bit American Standard Code for Information Interchange (ASCII) character. Therefore, conversion is performed from decimal ASCII characters to 8-bit integer values, conversion chart is shown in Table 7.1. Data

Figure 7.13: Communication Packet Frame

Table 7.1: Hexadecimal Notation for ASCII Decimal Characters to 8-bit Integers

\begin{tabular}{|c||c||c|}
\hline Data in Hexadecimal & ASCII Character & 8-bit integer value \\
\hline \hline $0 \times 30$ & 0 & $0 \times 00$ \\
\hline $0 \times 31$ & 1 & $0 \times 01$ \\
\hline $0 \times 32$ & 2 & $0 \times 02$ \\
\hline $0 \times 33$ & 3 & $0 \times 03$ \\
\hline $0 \times 34$ & 4 & $0 \times 04$ \\
\hline $0 \times 35$ & 5 & $0 \times 05$ \\
\hline $0 \times 36$ & 6 & $0 \times 06$ \\
\hline $0 \times 37$ & 7 & $0 \times 07$ \\
\hline $0 \times 38$ & 8 & $0 \times 08$ \\
\hline $0 \times 39$ & 9 & $0 \times 09$ \\
\hline
\end{tabular}

The flowchart in Figure 7.14 shows microcontroller received data packet processing methods. The $\mathrm{C}$ code implementing the algorithm is provided in Appendix E. 


\section{MATLAB GUI mode}

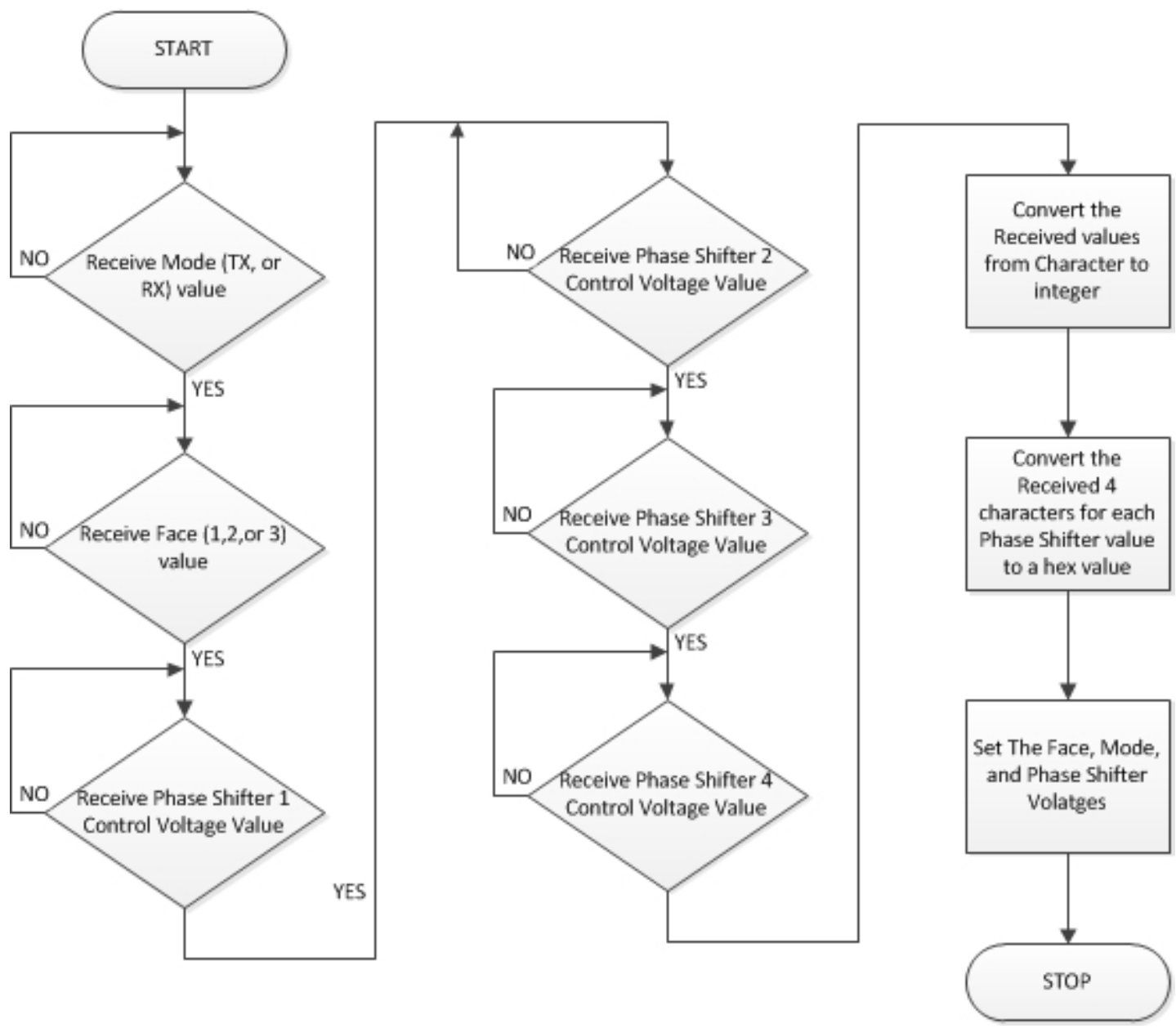

Figure 7.14: Microcontroller, MATLAB GUI Mode Flowchart

\subsection{Test and Results}

The board was initially tested with the RF system disconnected. Status LEDs were checked for SPDT and SP3T control lines. Set line or logic high is indicated by an illuminated LED; clear line or logic low is shown by a dark LED. The highlighted box in Figure 7.15 shows status LED locations. The phase shifter control voltages were measured using a digital multimeter. Table 7.2 shows the 
measured control voltages for three test cases:

1. $\theta=-30^{\circ} \phi=0^{\circ}$

2. $\theta=0^{\circ} \phi=0^{\circ}$

3. $\theta=15^{\circ} \phi=0^{\circ}$

Radiation patterns were collected for antenna Face-3 in transmit mode. The radiation pattern plots are shown in Figure 7.16. The offset angle in all the radiation patterns is explained in [1].

Table 7.2: GUI Test Result

\begin{tabular}{|c|c|c|c|c|}
\hline Theta & Phi & $\begin{array}{l}\text { Expected Phase } \\
\text { Shifter Control } \\
\text { Voltage }(\mathrm{V})\end{array}$ & $\begin{array}{l}\text { Measured Phase } \\
\text { Shifter Control } \\
\text { Voltage }(\mathrm{V})\end{array}$ & Percent Error \\
\hline \multirow[t]{4}{*}{-30} & 0 & 7.98 & 7.96 & $-0.25 \%$ \\
\hline & & 7.98 & 7.94 & $-0.50 \%$ \\
\hline & & 0 & 0.01 & NA \\
\hline & & 0 & 0.01 & NA \\
\hline \multirow[t]{4}{*}{0} & 0 & 0 & 0.01 & NA \\
\hline & & 0 & 0.01 & NA \\
\hline & & 0 & 0.01 & NA \\
\hline & & 0 & 0.01 & NA \\
\hline \multirow[t]{4}{*}{15} & 0 & 0 & 0.01 & NA \\
\hline & & 0 & 0.01 & NA \\
\hline & & 5.64 & 5.62 & $-0.35 \%$ \\
\hline & & 5.64 & 5.61 & $-0.53 \%$ \\
\hline
\end{tabular}




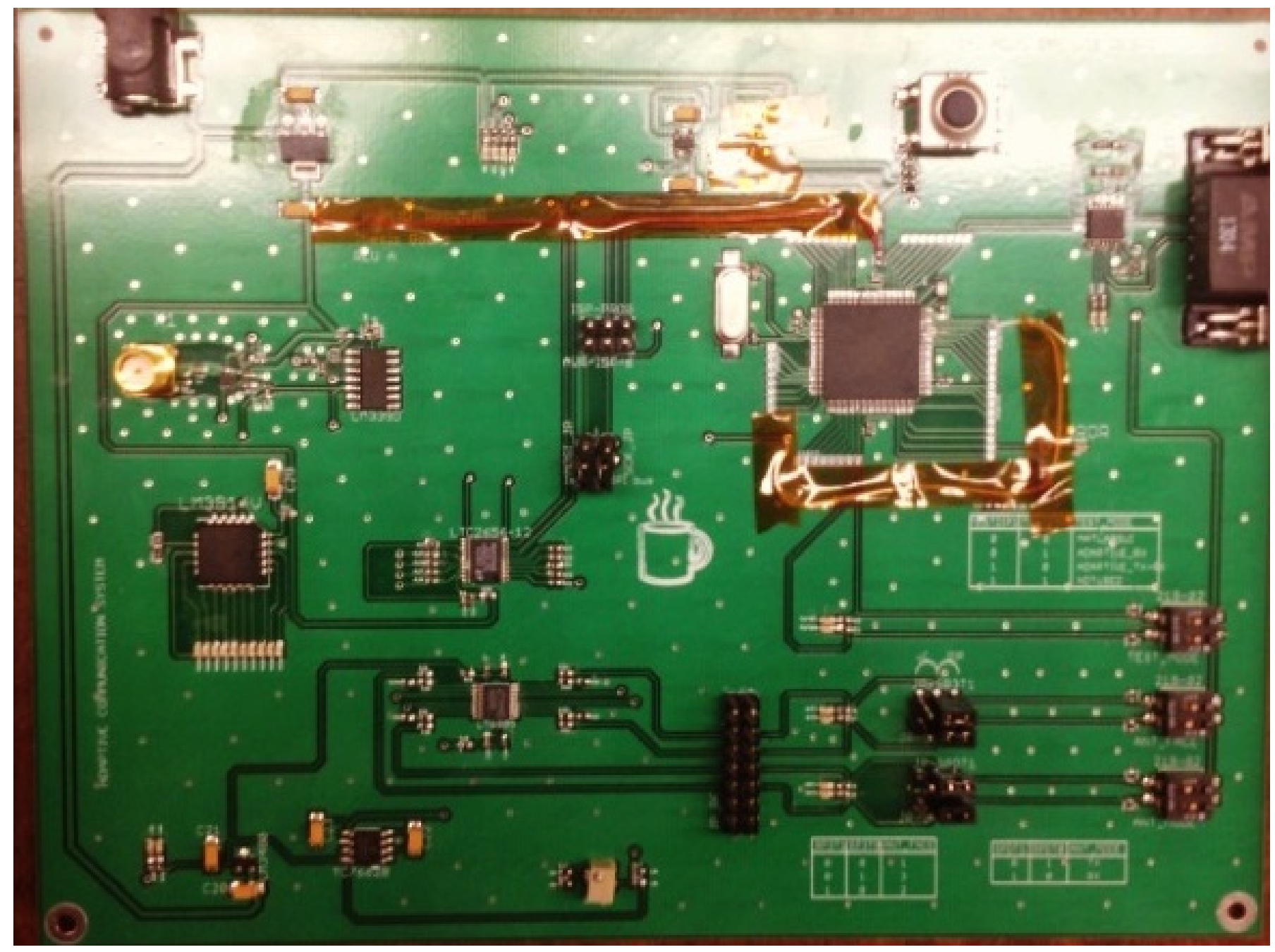

Figure 7.15: Status LED's for Mode and Face value 


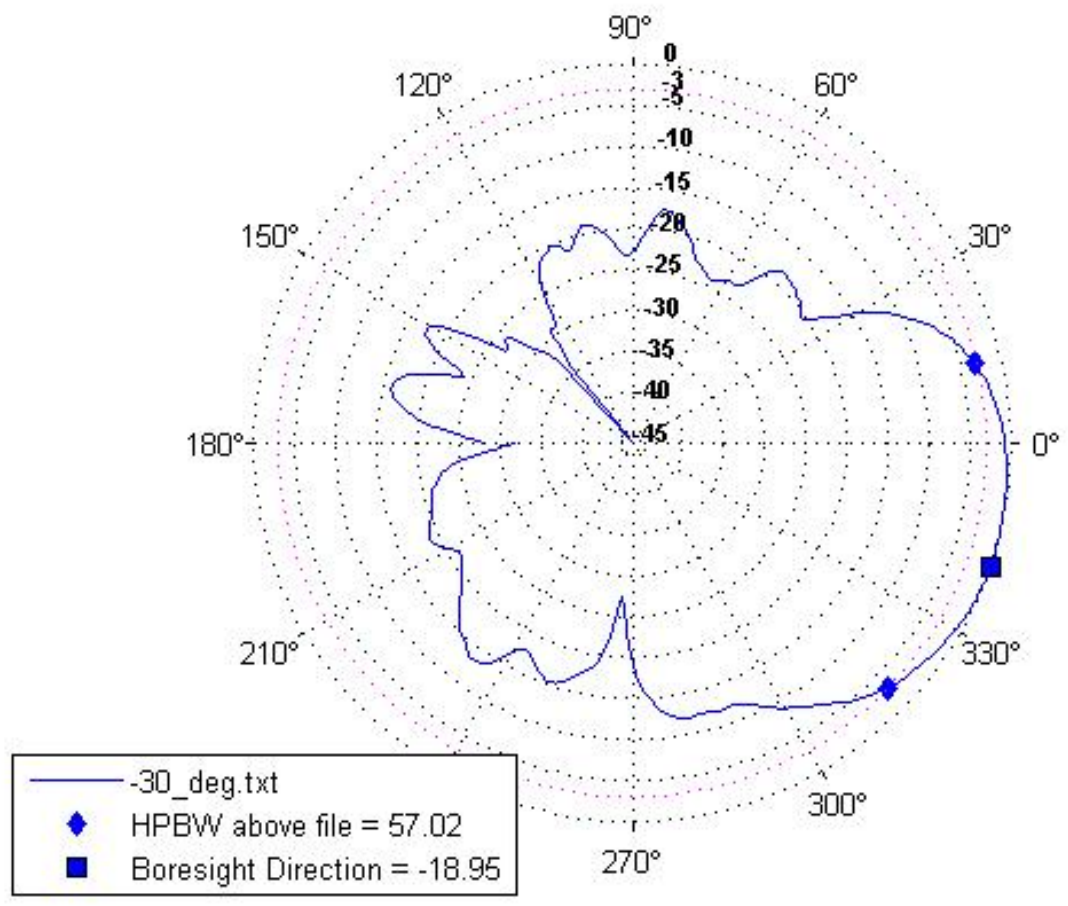

(a) Radiation Pattern, $\theta=-30^{\circ} \phi=0^{\circ}$

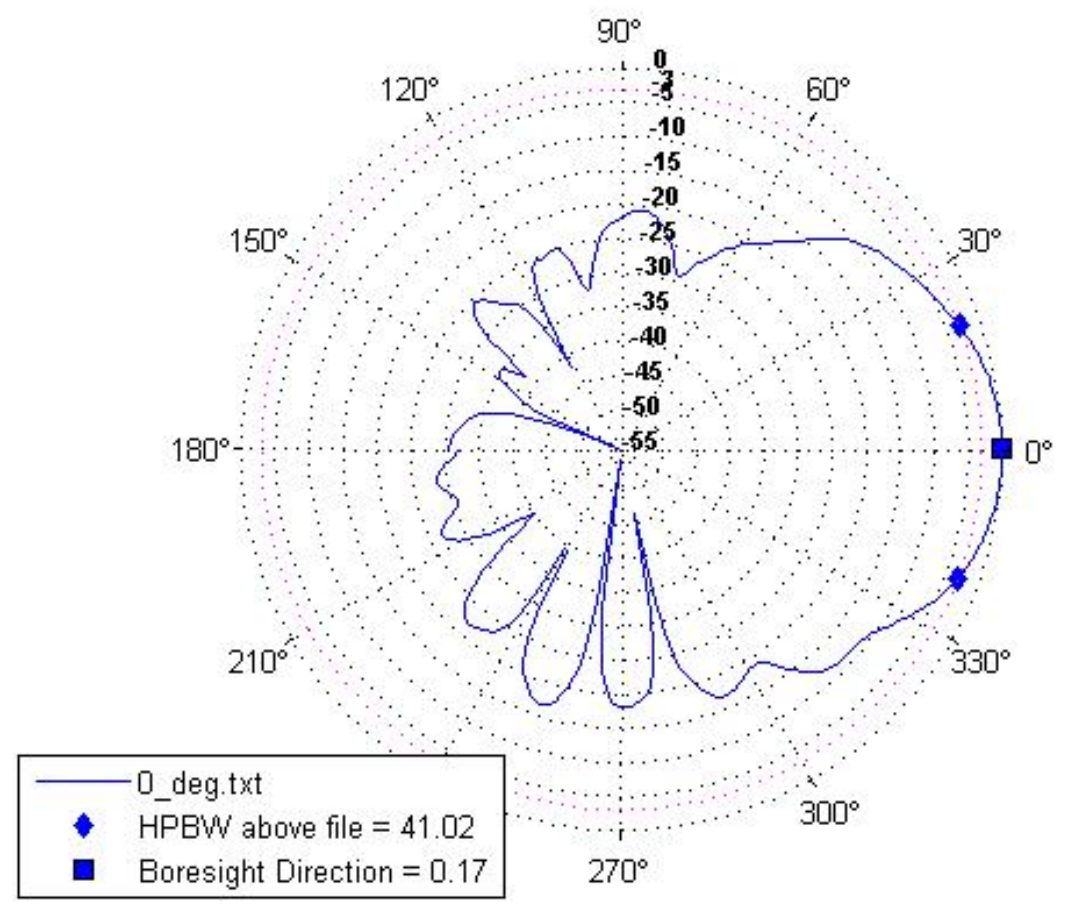

(b) Radiation Pattern, $\theta=0^{\circ} \phi=0^{\circ}$

Figure 7.16: GUI Test Measured Radiation Patterns 


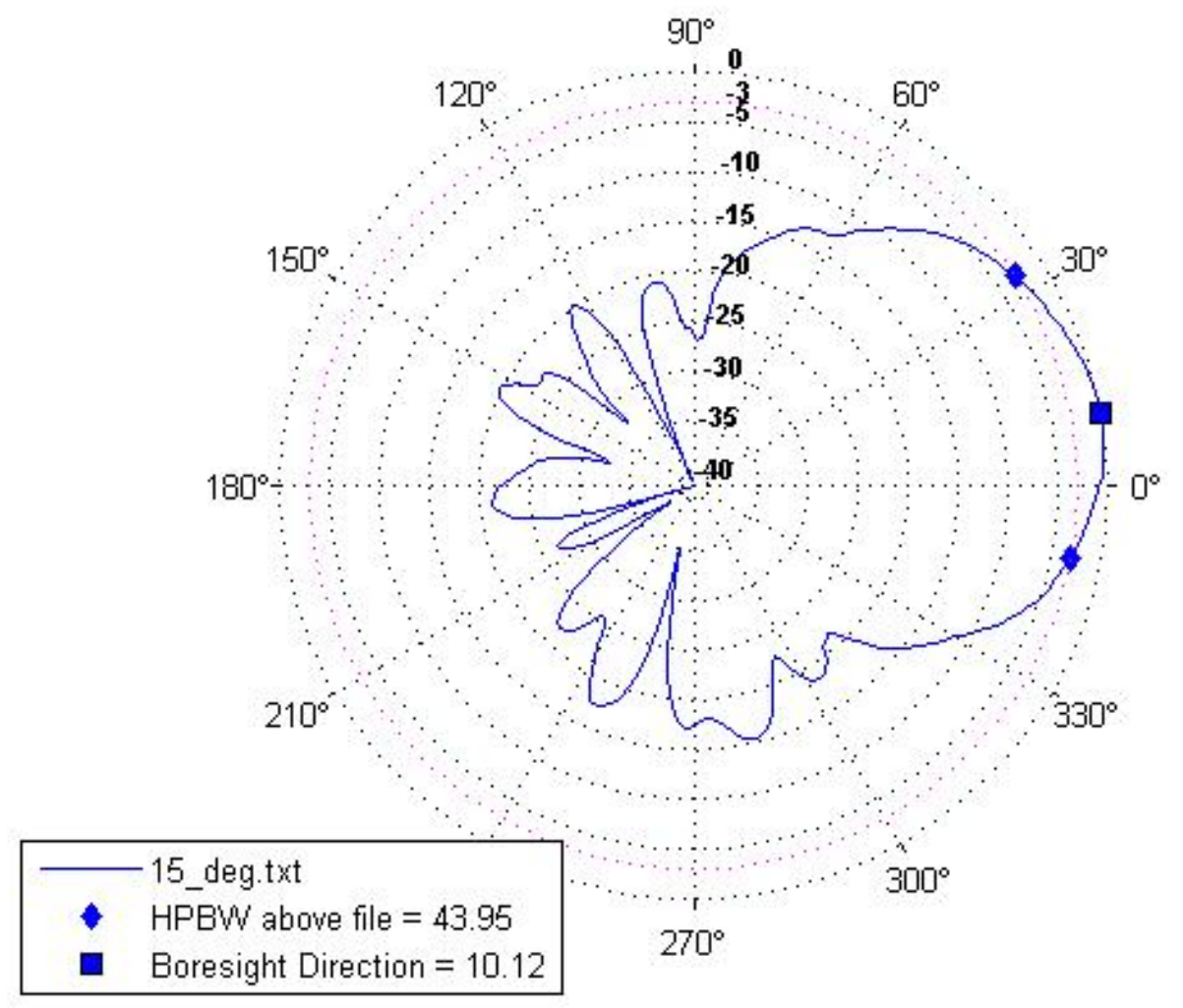

(c) Radiation Pattern, $\theta=15^{\circ} \phi=0^{\circ}$

Figure 7.16: GUI Test Measured Radiation Patterns 


\section{CHAPTER 8}

\section{ADAPTIVE RECEIVE MODE}

In receive mode, an adaptive antenna performs a scan to determine the maximum receive power direction. This mode to used to maintain a lock on a receiving signal from a transmitting antenna.

\subsection{Adaptive Receive Algorithm}

For the search algorithm, a 58-point scan on each antenna face is performed. Figure 8.1 shows the location of the points used in the the adaptive receive scan. The points are scanned using a raster scan technique, which is a line-by-line rectangular sweep as shown in Figure 8.2. Power is received for each scan point. If the received power at the current point is greater than the power received at any previous point, the new maximum is stored. This continues until the scanning is complete. Following scan completion, the beam is reset to the maximum power direction. The scan direction is maintained until the receive power level decreases below $-50 \mathrm{dBm}$. This level is chosen because the RF system has a noise floor of $-60 \mathrm{dBm}$. 


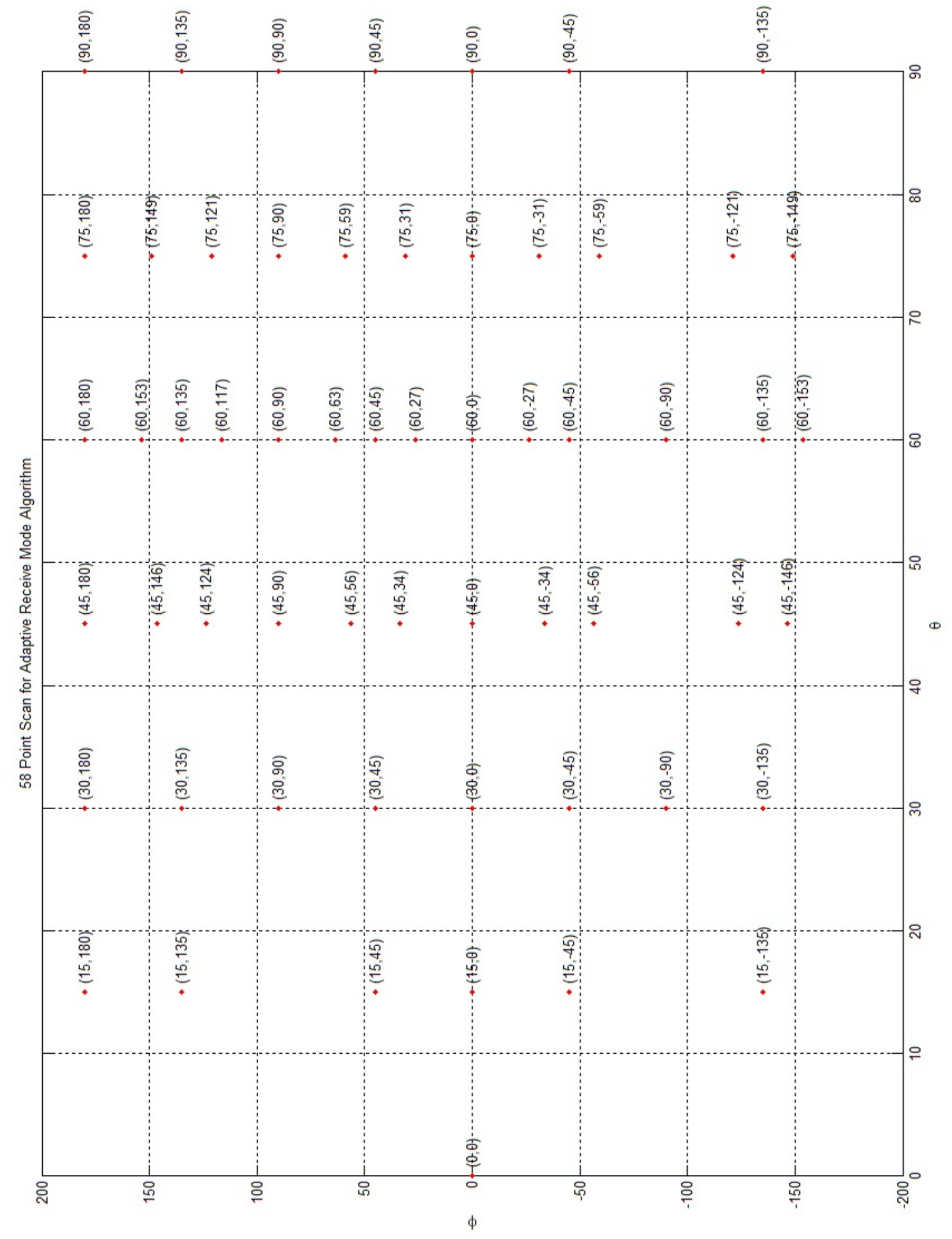

Figure 8.1: Adaptive Receive scan plot 


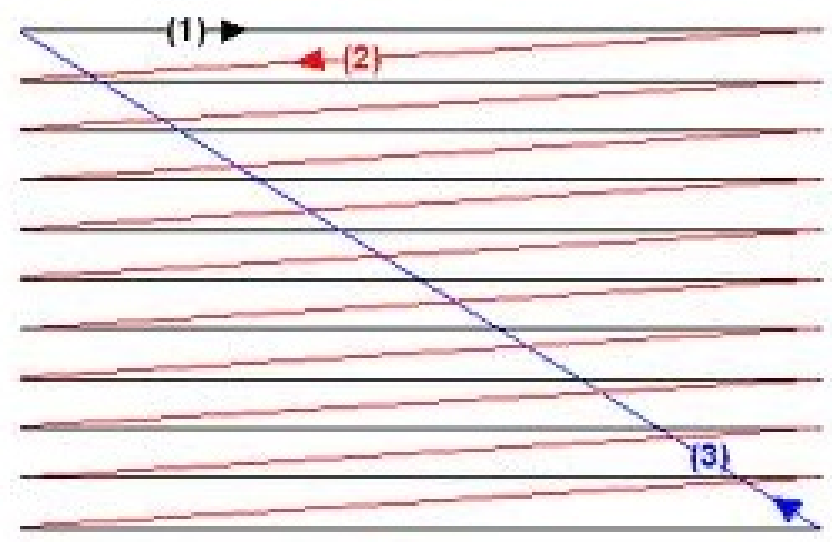

Figure 8.2: Raster Scan Pattern

The flowchart in Figure 8.3 shows the scan algorithm in the adaptive receive mode. Upon scan completion, if the lock flag is set, the maximum beam direction is initialized. If the flag was not set in the previous scan, the scan repeats itself. The lock flag is initially set when the receive power is greater than $-50 \mathrm{dBm}$, or subsequently if the received power is greater than the previously received maximum power. The interrupt service routine (ISR) flowchart for the microcontroller is shown in Figure 8.4. Figure 8.4 shows the flowchart for the interrupt service routine (ISR), which is triggered when the comparator output changes to logic high. 


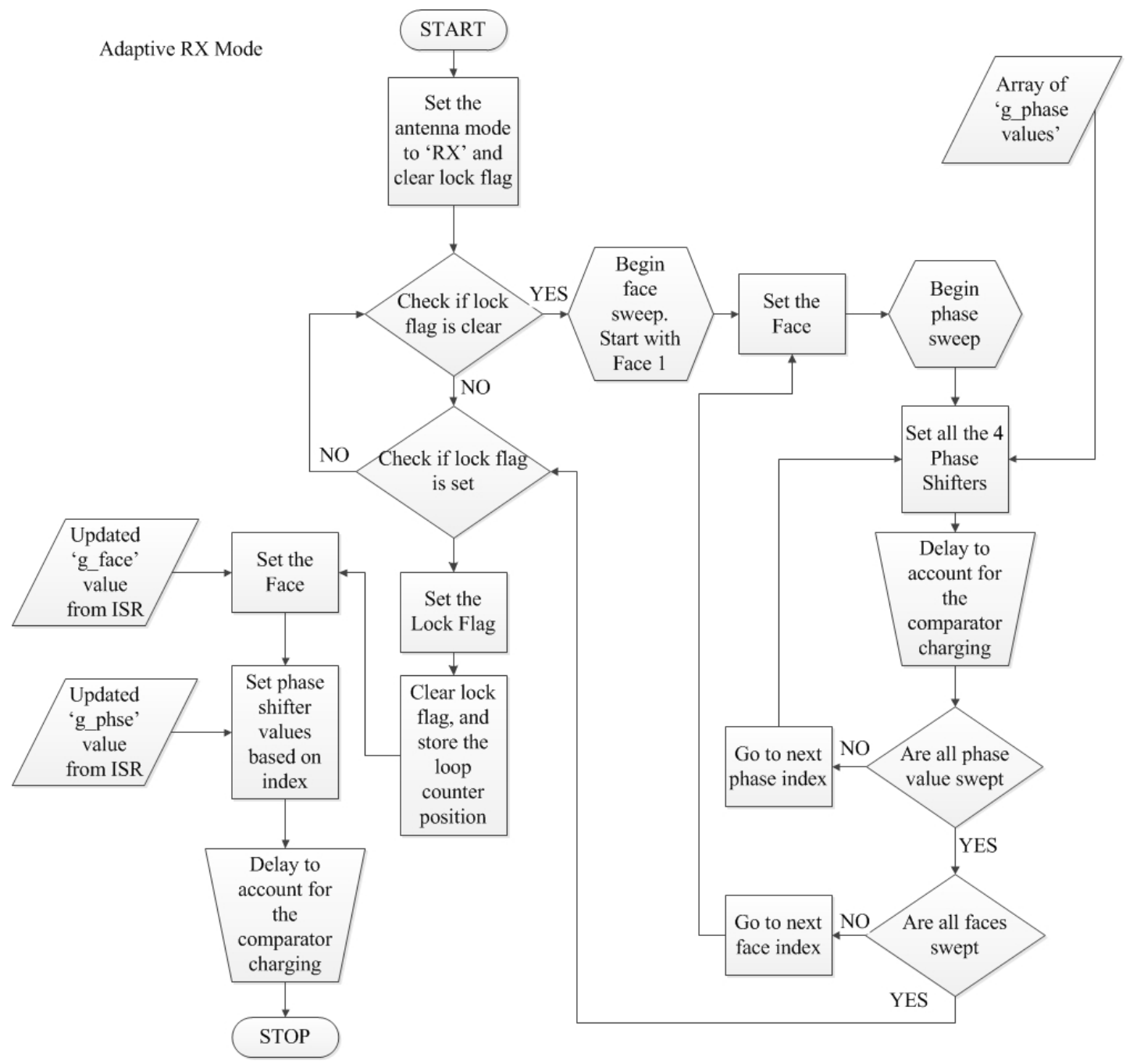

Figure 8.3: Adaptive Receive Flowchart 


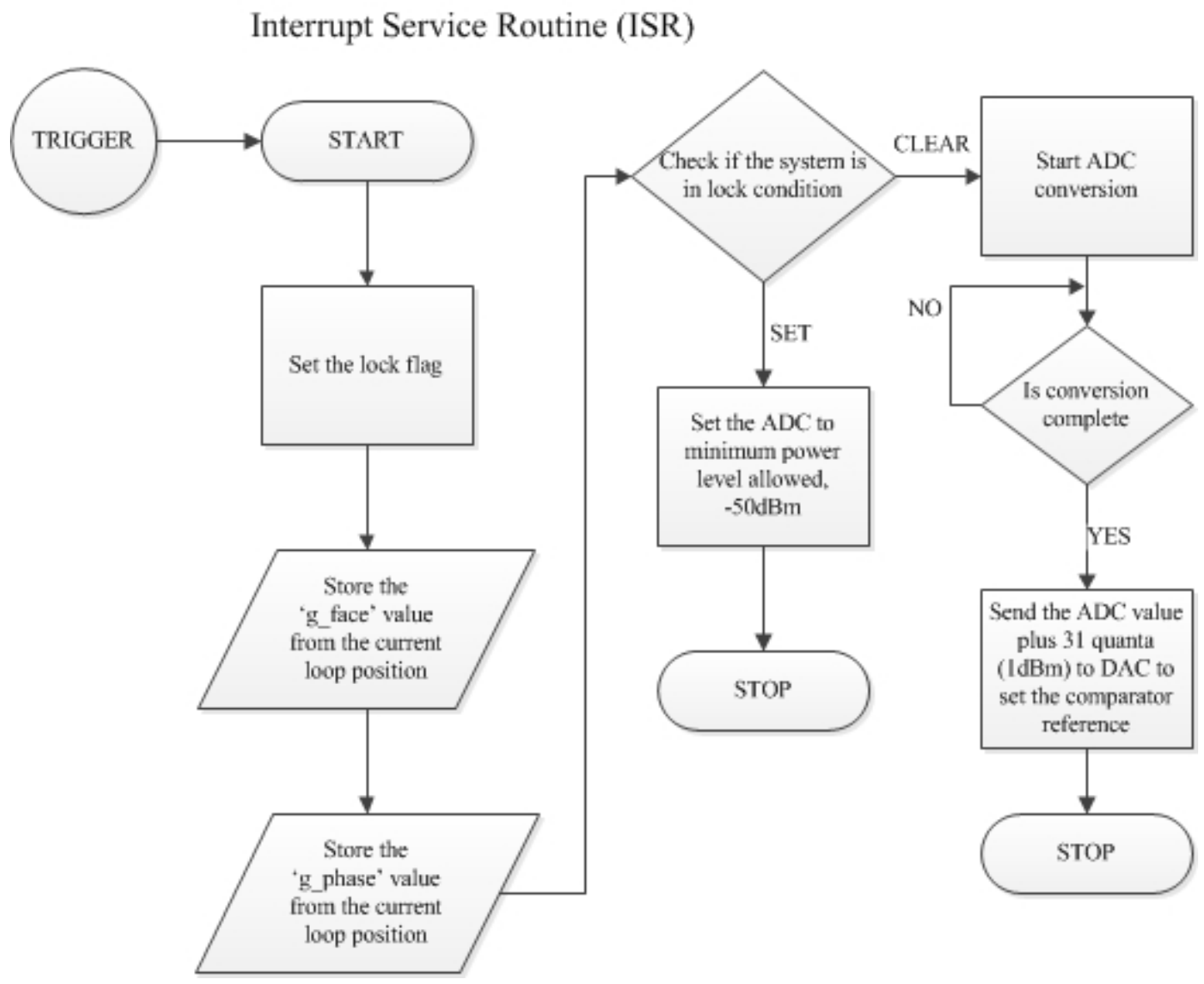

Figure 8.4: ISR Flowchart

\subsection{Testing}

The system was tested without the RF board and antenna connected to the digital control board. A Wiltron (Model 68147A) RF source was used to simulate the received power. A delay of $100 \mathrm{~ms}$ was set between scan points to facilitate observation of the working algorithm. Table 8.1 shows adaptive receive mode test procedure data. The following test procedure was performed:

1. Apply $-60 \mathrm{dBm}$ power to the receive port

2. Change the power to $-30 \mathrm{dBm}$ at a single scan point and then back to 
$-60 \mathrm{dBm}$, to set the trigger

3. Observe when Lock LED status turns on

4. Revert back to -30dBm power

5. Reduce the power from $-30 \mathrm{dbm}$ in $2 \mathrm{~dB}$ steps and observe lock LED turn off once $-50 \mathrm{dBm}$ is reached

Table 8.1: Test Data for Adaptive Receive Mode Test Procedure

\begin{tabular}{|c|c|}
\hline Receive Power (dBm) & Lock LED status \\
\hline-30 & $\mathrm{ON}$ \\
\hline-32 & $\mathrm{ON}$ \\
\hline-34 & $\mathrm{ON}$ \\
\hline-36 & $\mathrm{ON}$ \\
\hline-38 & $\mathrm{ON}$ \\
\hline-40 & $\mathrm{ON}$ \\
\hline-42 & $\mathrm{ON}$ \\
\hline-44 & $\mathrm{ON}$ \\
\hline-46 & $\mathrm{ON}$ \\
\hline-48 & $\mathrm{ON}$ \\
\hline-50 & $\mathrm{OFF}$ \\
\hline-52 & $\mathrm{OFF}$ \\
\hline-54 & $\mathrm{OFF}$ \\
\hline-56 & $\mathrm{OFF}$ \\
\hline-58 & $\mathrm{OFF}$ \\
\hline-60 & $\mathrm{OFF}$ \\
\hline
\end{tabular}


The data in Table 8.1 proves that system operates in the specification, maintaining lock for power level greater than $-50 \mathrm{dBm}$. The complete system test was not performed due to RF PCB manufacturing problems [1]. 


\section{CHAPTER 9}

\section{CONCLUSIONS AND FUTURE WORK}

The system shows a successful implementation of the digital control board for beam steering and adaptive communications. The digital control board controls the antenna face and mode as described in [1]. Each sub-block of the digital controlled board was tested in order to verify that specifications were met. The MATLAB graphical user interface successfully controlled the digital control board. An adaptive communication system was implemented to demonstrate a working example. The system autonomously locked to the maximum receive power using the 58-point scan, for the power levels above $-50 \mathrm{dBm}$.

The RF power detector used a logarithmic amplifier-based design, which provides a linear relation between input power and output voltage. The RF power detector has a slope of $37.3 \mathrm{mV} / \mathrm{dB}$ with a minimum detectable power of $-60 \mathrm{dBm}$ and maximum detectable power of $0 \mathrm{dBm}$.

An ATmega2560 is used as the microcontroller for the system. The built-in ADC was used to measure the receive power. The microcontroller is also used to communicate with MATLAB, which allows it to set the output voltages for the phase shifters as well as the control signals that set the antenna face and the mode. The microcontroller can be configured in MATLAB GUI mode or the adaptive receive mode.

The system successfully controls the SP3T and SPDT switches, which control 
active antenna face and mode selection, respectively. The system also successfully sends control voltages to the phase shifter within $0.53 \%$ of expected values. The control board demonstrates a working bench test for an adaptive receive station.

The PCB design shows improved performance with a separate analog and digital plane and reduces the noise on the analog signal. The digital control board was designed for in system test and debugging. The digital control board has features for future expandability.

Future work:

- Re-test the digital control board with a fully operational RF board

- Develop a half duplex adaptive receive and transmit communication system

- Digital communication (16-QAM, APSK, LTE, etc.) implementation on the control board 


\section{BIBLIOGRAPHY}

[1] S. Brockhoff, "Electronically-Steered Tetrahedral-Structured Phased Array 2.4 GHz Transceiver Design Applied to Adaptive Communications," Master's thesis, California Polytechnic State University, 2013.

[2] Louis E. Frenzel, "Design FAQ: RF Detector for Wireless Devices." http : //www.analog.com/static/imported files/faqs/201551981Detector ${ }_{F} A Q . p d f$.

[3] "Integrated DC Logarithmic Amplifiers," Dallas Semiconductor, Maxim Engineering Journal, vol. 56, 2005.

[4] "LT5534 - 50MHz to 3GHz RF Power Detector with 60dB Dynamic Range." http : //www.linear.com/product/LT5534.

[5] "LT5538 - 40MHz to $3.8 \mathrm{GHz}$ RF Power Detector with 75dB Dynamic Range." http://www.linear.com/product/LT5538.

[6] "LT5534 Datasheet." http ://cds.linear.com/docs/en/datasheet/5534fc.pdf.

[7] "LT5534 Demo Board." http : //www.linear.com/demo?demoboard = part $_{n}$ umber $=5534$ category $y_{i} d=0$ companion $_{b}$ oard $=$ software $_{i} d=$.

[8] "LT5538 Datasheet." http ://cds.linear.com/docs/en/datasheet/5538f.pdf.

[9] "LT5538 Demo Board." http : //www.linear.com/demo?demoboard = part $_{n}$ umber $=5538$ category $y_{i} d=0$ companion $_{b}$ oard $=$ software $_{i} d=$. 
[10] Morris R. Driels and Young S. Shin, "DETERMINING THE NUMBER OF ITERATIONS FOR MONTE CARLO SIMULATIONS OF WEAPON EFFECTIVENESS," Tech. Rep. NPS-MAE-04-005, Naval Postgraduate School, MONTEREY, CALIFORNIA, April 2004. 


\section{APPENDICES}

A 2013 IEEE APS Student Design Challenge: Reconfigurable Antennas 


\title{
- IEEE
}

\section{IEEE AP-S Student Design Challenge: Reconfigurable Antennas}

\author{
Travel to the 2013 IEEE Antennas and Propagation Symposium \\ Win \$1500, \$750, \$250
}

Join the $4^{\text {th }}$ IEEE AP-S Antenna Design Challenge! Build an antenna system with reconfigurable antenna elements that can adapt to different propagation conditions (e.g. line-of-sight versus non-line-of-sight). The top three teams will receive up to $\$ 2,500$ (US dollars) in travel funds to attend the IEEE Antennas and Propagation Symposium in Orlando, FL, USA, July 7-13, 2013 to demonstrate their working systems. From these top teams, first, second, and third place winners will be announced at the 2013 IEEE AP-S Awards Banquet and will receive cash awards of $\$ 1500, \$ 750$, and $\$ 250$, respectively.

Important deadlines are November 30, 2012 and April 26, 2013. See below for details.

\section{Goals and Specifications:}

1. Design an antenna system with reconfigurable antenna elements that can adapt to different propagation conditions in order to achieve the best link performance. The performance can be shown in terms of received power or higher level system metric such as symbol error rate.

2. The antenna system should be used to teach how antennas work. Such a system might be used in college undergraduate or graduate courses, and in pre-college ('High School') physics courses.

3. The system must be safe and durable, easily reproducible by others, inexpensive, and portable so that it can be demonstrated at the Symposium.

4. The system must operate at $2.4 \mathrm{GHz}$, have its own source (i.e. no commercial signal generator can be used), and fit on a table top (roughly 24"x $40^{\prime \prime}$ ) or two closely spaced tables. Readily available software (e.g., student versions of C, Matlab, Visual Basic, LabView) or free software packages may be used. All software must be included in the budget.

5. The total cost for reproduction of the system must be less than $\$ \mathbf{1 , 5 0 0}$. The use of a laptop computer is allowed and does not have to be included in the $\$ 1,500$ limit.

\section{Eligibility:}

The team should consist of 2 to 5 persons, with a majority of undergraduate students. Each team should be advised by a professional mentor who is a member of the AP Society. No student or mentor should be involved in more than one team.

\section{The Application and Review Process:}

1. All applicants must submit a preliminary design by November 16, 2012. It must include:

a. A proposal limited to two pages and in 12-pt Times New Roman font that includes

i. A detailed description of the system to be built. 
ii. The steps that will be taken to ensure the accuracy of the system.

iii. A bill of materials (up to $\$ 1,500$ ).

b. A letter from a professional mentor such as a professor or engineer in industry indicating agreement to supervise the project. The mentor must be an IEEE AP-S member and must verify that all members of the team are graduate and/or undergraduate students at a university, college, or technical school. The proposal and letter must be integrated into a single pdf file named TeamName.pdf. The proposal should precede the letter.

2. The Design Contest Committee will assess each preliminary design based on creativity, likelihood of achieving the design goals, educational value, and quality of written materials. Six semi-finalist teams will be selected by November 30, 2012 and will receive \$1,500 each to build and test their designs.

3. Each of the six semi-finalist teams must submit its final design by April 26, 2013. It must be accompanied by a video demonstration of its working system, and a final report in pdf format using the template available at the AP-S web-site. Submission instructions for the video demonstration will be provided later. The report must be limited to 10 pages and include:

a. A detailed description of the system (including schematic and other diagrams).

b. A list of parts and materials required, including where to obtain them and costs.

c. Photos of the final system (including a scale to show how large it is).

d. Assembly and operating instructions for the system.

e. A set of measurements obtained using the system with an example test antenna.

f. Biographies (100 words or less each) and photos of all design team members.

g. All software necessary to build and/or operate the system in a separate file(s).

4. The Design Contest Committee will assess each semi-finalist's design based on creativity, completeness of the description, functionality of the system as determined by the video, educational value, and quality of written materials. Three finalist teams will be selected by May 10, 2013 and will receive stipends of up to $\$ 2,500$ per team to travel to and attend the IEEE AP-S Symposium. The stipend is intended to cover equipment shipping costs and all of the expenses for one team representative; however, it may be divided among multiple team members.

5. The finalists will be expected to demonstrate their working systems during the Symposium and attend the Awards Ceremony at the banquet. Power supplies will be made available at the Symposium. Each team is responsible for bringing all other necessary equipment for a successful demonstration.

6. The Design Contest Committee will judge the final demonstrations and select the first, second, and third prize winners to receive $\$ 1,500, \$ 750$ and $\$ 250$, respectively. The prize winners will be announced at the AP Awards Banquet.

7. After the Symposium, the finalists will be required to revise the final report for publication in the IEEE AP Magazine under the Education Column.

\section{How to Submit Materials:}

Send all materials to designcontest@ieeeaps.org with the subject line "2013 IEEE AP-S Design Contest Submission." Questions may be sent to the same address. All submitted materials must be in pdf format according to the guidelines above. 
B Contest Proposal 


\section{IEEE Antenna Design Contest By: Steve Brockhoff, Mayur Bansal}

\section{Objective}

We plan to build an electrically reconfigurable antenna system to demonstrate line-of-sight and non-line-of-sight transmission. The physical structures of both transmit and receive antenna systems will be a triangular-based pyramidal structure. Each face of the structure has three probe-fed triangular microstrip patch antennas See Figure 1 for the general structure.

This structure has two levels of precision. The face and patch antenna arrangement creates a three dimensional phased array in which array elements are phase-controlled to direct a beam to three-dimensional sectors. Two elements on one face could be

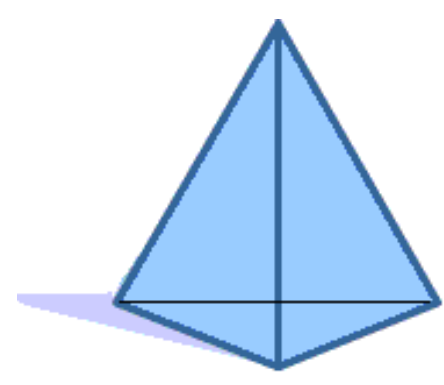

Figure 1: Triangular-based pyramidal structure. activated and one on another to optimize beam-steering to maximize signal reception.

Transmit and receive antenna systems are completely isolated and are identical, both capable of transmitting and receiving. Two-way communication enables transmission beam directing to maximize receive antenna reception.

\section{Preliminary Design}

The 2.4 GHz signal is generated using an Analog Devices integrated synthesizer (CC2500). Three $2.4 \mathrm{GHz}$ amplifiers amplify signals for transmission and after reception. Three $2.4 \mathrm{GHz}$ bandpass filters suppress out-of-band power by at least $10 \mathrm{~dB}$. Each bandpass filter will be a cascaded combination of Chebyshev low-pass and high-pass filters with cutoff frequencies of $2.35 \mathrm{GHz}$ and $2.45 \mathrm{GHz}$, respectively. Amplifier placement before the filters minimizes noisefigure.

The signal is applied to a distribution network, which includes four phase-shifters controlled by an Arduino microcontroller, see Fig. 2. The Arduino controls the antenna and active face by controlling RF switches in order coarsely scan for the optimal transmit direction. Multiple faces can be active at one time. The design utilizes three phase-shifters to control three patch antennas on

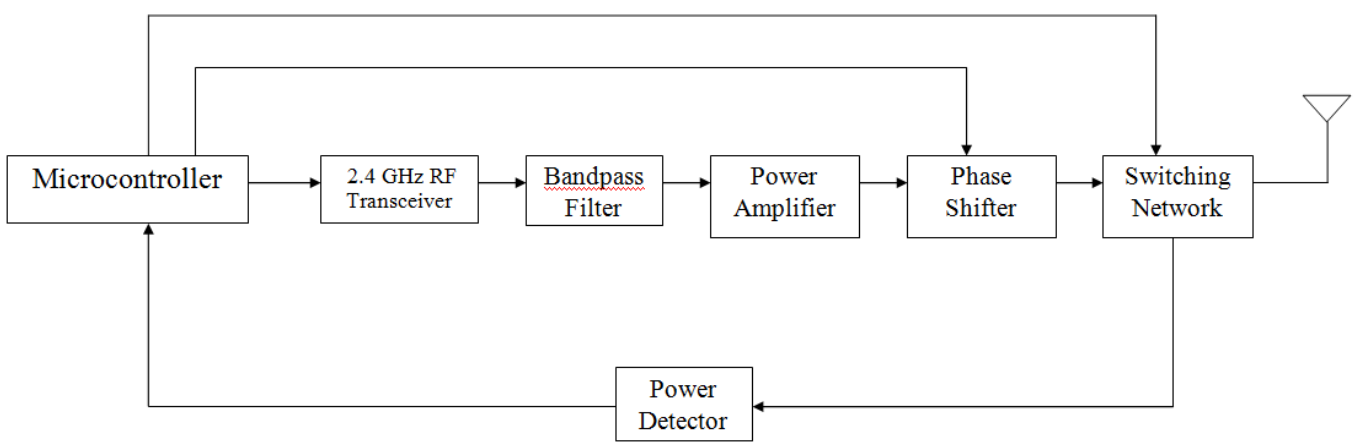

Figure 2: System Block Diagram 
different structure faces. Three of the nine patch antennas will be active at any one time.

To maximize received signal strength, the microcontroller selects combinations of faces and active patches to direct the transmit signal. Concurrently, the receive antenna scans in a similar fashion in a two-step process. First, all phase-shifters are set to zero and the signal is applied to different three-element combinations of the TX patch antennas. The system then waits for an acknowledgement (ACK) signal from the RX antenna. The TX system measures the ACK signal power and saves it in the Arduino memory and associates the recorded power level with the specific three-element combination. Second, the Arduino recalls each three-element combination, then controls the phase-shifters to change the beam direction to finely scan for the optimal transmit direction. The TX antenna waits for an ACK signal, as before, but will now store the phaseshift values in memory as well, in order to recall the coarse and fine directions for optimal transmission.

The first scan determines the general direction to establish a link (both the TX and RX directions)Then, the phase-shifters are adjusted to finely scan in each direction that received an ACK message, starting with the strongest sector direction. After all antenna combinations and fine scanning is completed, the three-element array and phase-shift values that correspond to the maximum signal strength is chosen as the optimum transmission direction.

\begin{tabular}{|c|c|c|c|c|c|c|}
\hline \multirow{3}{*}{$\begin{array}{l}\text { Component } \\
\text { Antenna }\end{array}$} & Part & Unit Price & Qty & \multicolumn{2}{|c|}{ Cost } & \multirow{2}{*}{$\begin{array}{l}\text { Notes } \\
\text { PCBExpress }\end{array}$} \\
\hline & PCB & $\$ \quad 45.00$ & 6 & $\$$ & 270.00 & \\
\hline & SMA Connector & 2.50 & 9 & $\$$ & 22.50 & DigiKey \\
\hline \multirow[t]{12}{*}{ Motherboard } & PCB & 75.00 & 2 & $\$$ & 150.00 & PCBExpress \\
\hline & Microcontroller & 30.00 & 2 & $\$$ & 60.00 & Arduino \\
\hline & Crystal & 0.35 & 2 & $\$$ & 0.70 & Analog Device \\
\hline & Transreciever & 4.76 & 2 & $\$$ & 9.52 & Texas Instruments \\
\hline & Power detector & 19.42 & 2 & $\$$ & 38.84 & Analog Device \\
\hline & RF Amp & 1.50 & 20 & $\$$ & 30.00 & Analog Device \\
\hline & Active Phase Shifter & 5.35 & 8 & $\$$ & 42.80 & Mini Circuits \\
\hline & RF Switch ( SP3T) & 8.36 & 42 & $\$$ & 351.12 & HMC245QS16 \\
\hline & RF Switch ( SP2T) & 0.52 & 18 & $\$$ & 9.36 & AS179 \\
\hline & R,L,C,D & & & $\$$ & 50.00 & DigiKey \\
\hline & LCD $16 X 2$ character & 13.95 & 2 & $\$$ & 27.90 & Sparkfun \\
\hline & SMA Connector & 2.00 & 18 & $\$$ & 36.00 & DigiKey \\
\hline \multirow{3}{*}{$\begin{array}{l}\text { Accessories } \\
\text { Total }\end{array}$} & SMA male to male cable & 12.96 & 18 & $\$$ & 233.28 & DigiKey \\
\hline & MATLAB Student Version & $\$ 100.00$ & 1 & $\$$ & 100.00 & \\
\hline & & & & \multicolumn{2}{|c|}{$\$ 1,432.02$} & \\
\hline
\end{tabular}


C LT 5534 Demo Board Schematic 


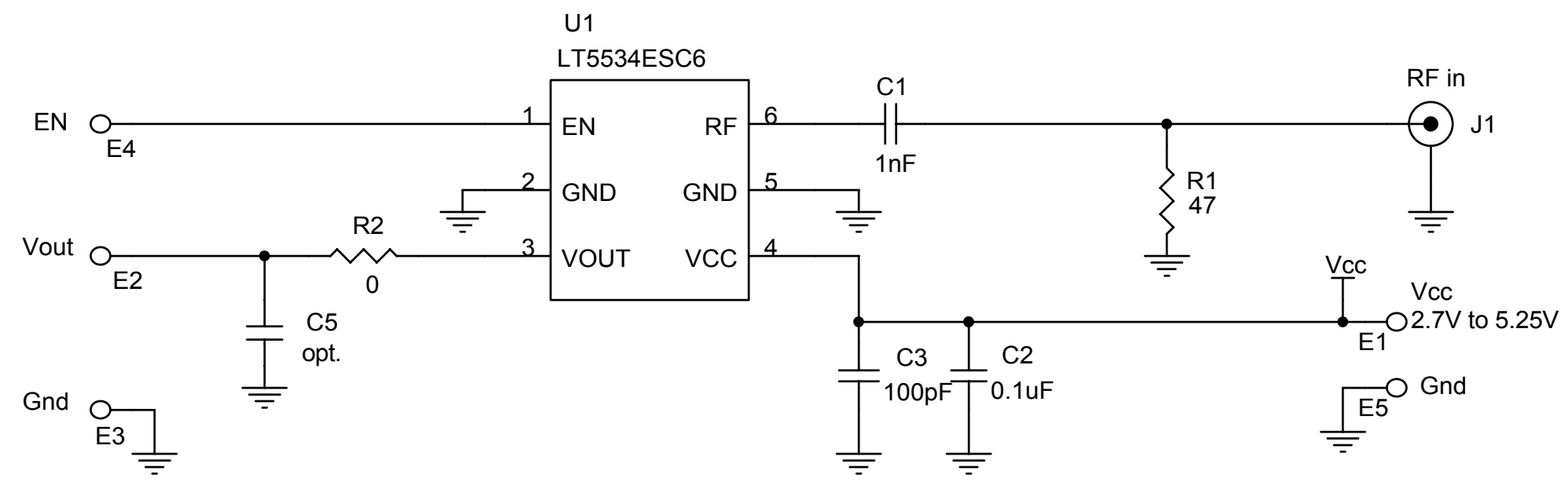

\section{CUSTOMER NOTICE}

LINEAR TECHNOLOGY HAS MADE A BEST EFFORT TO DESIGN A CIRCUIT THAT MEETS CUSTOMER-SUPPLIED SPECIFICATIONS; HOWEVER, IT REMAINS THE CUSTOMER'S RESPONSIBILITY TO VERIFY PROPER AND RELIABLE OPERATION IN THE ACTUAL APPLICATION. COMPONENT SUBSTITUTION AND PRINTED CIRUIT BOARD LAYOUT MAY SIGNIFICANTLY AFFECT CIRCUIT PERFORMANCE OR RELIABILITY. CONTACT LINEAR

TECHNOLOGY APPLICATIONS ENGINEERING FOR ASSISTANCE.

THIS CIRCUIT IS PROPRIETARY TO LINEAR TECHNOLOGY AND SUPPLIED FOR USE WITH LINEAR TECHNOLOGY PARTS.

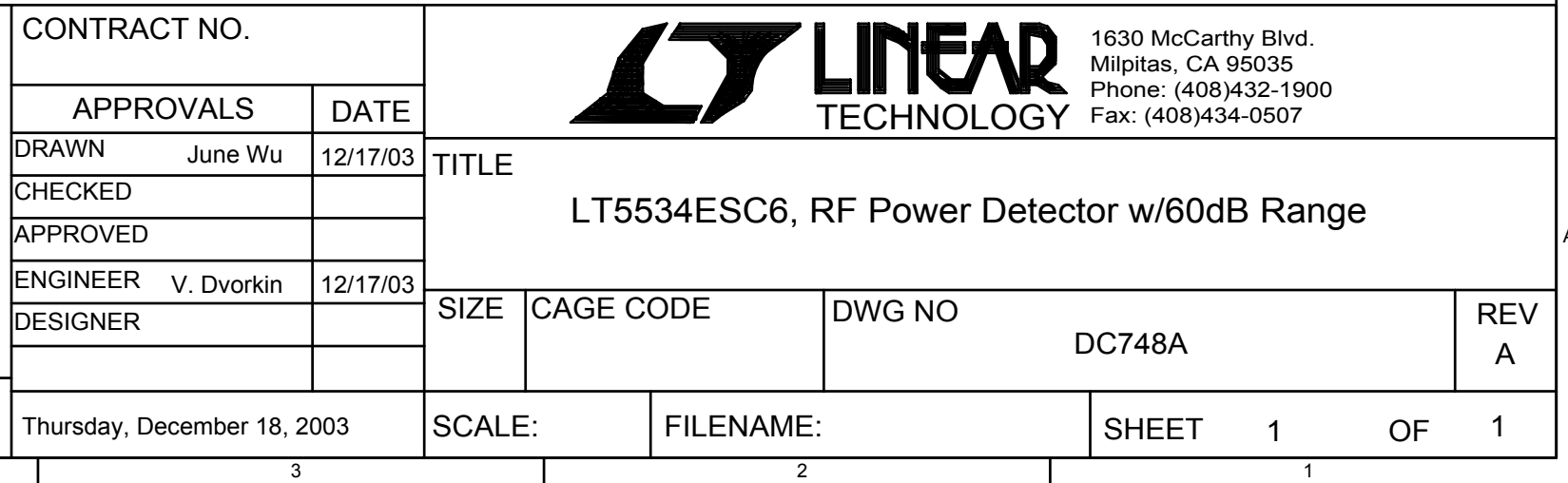




\section{LT 5538 Demo Board Schematic}




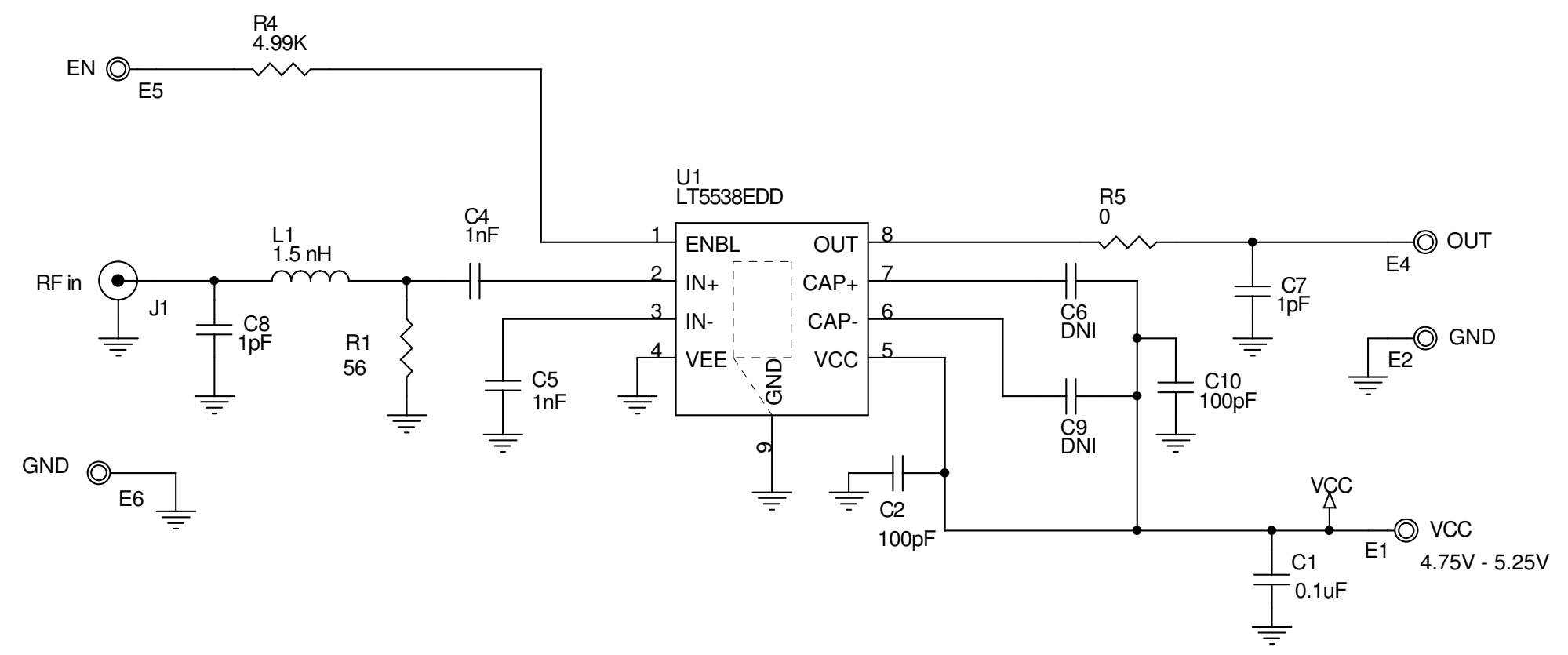

\begin{tabular}{|c|c|c|c|c|c|c|c|c|c|}
\hline & \multicolumn{2}{|l|}{ CONTRACT NO. } & \multirow{2}{*}{ TECHNOLOGY } & \multirow{2}{*}{\multicolumn{2}{|c|}{ 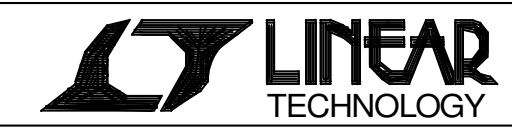 }} & \multirow{2}{*}{\multicolumn{4}{|c|}{$\begin{array}{l}1630 \text { McCarthy Blvd. } \\
\text { Milpitas, CA95035 } \\
\text { Phone: : } 4088432-1900 \\
\text { Fax: }(408) 434-0507\end{array}$}} \\
\hline & \multirow{2}{*}{\multicolumn{2}{|c|}{ APPROVALS }} & & & & & & & \\
\hline & $\begin{array}{|ll|}\text { DRAWN } & \text { A. Karpova } \\
\end{array}$ & & \multirow{3}{*}{ TITLE } & \multirow{3}{*}{\multicolumn{6}{|c|}{$\begin{array}{l}\text { LT5538EDD } \\
50 \mathrm{MHz} \text { to } 3.8 \mathrm{GHz} \text { DETECTOR }\end{array}$}} \\
\hline & \multirow{2}{*}{\multicolumn{2}{|c|}{\begin{tabular}{|l} 
APPROVED \\
\end{tabular}}} & & & & & & & \\
\hline & & & & & & & & & \\
\hline & \begin{tabular}{|ll} 
ENGINEER & D.Stuetzle \\
DESIGNER
\end{tabular} & $6 / 12 / 06$ & \multirow[t]{2}{*}{ SIZE } & \multirow[t]{2}{*}{ CAGE CODE } & \multirow{2}{*}{\multicolumn{4}{|c|}{ DC1120A }} & \\
\hline & DESIGNER & & & & & & & & 3 \\
\hline $\begin{array}{l}\text { CUSTOMER NOTICE } \\
\text { LINEAR TECHNOLOGY HAS MADE A BEST EFFORT TO DESIGNA } \\
\text { CIRCUIT THAT MEETS CUSTOMER-SUPPLIED SPECIFICATIONS; } \\
\text { HOWEVER, IT REMAINS THE CUSTOMER'S RESPONSIBILITY TO } \\
\text { VERIFY PROPER AND RELIABLE OPERATION IN THE ACTUAL } \\
\text { APPLICATION. COMPONENT SUBSTITUTION AND PRINTED } \\
\text { CIRUIT BOARD LAYOUT MAY SIGNIFCANTLY AFFECT CIRCUIT } \\
\text { PERFORMANCE OR RELIABILTY. CONTACT LINEAR } \\
\text { TECHNOLOGY APPLICATIONS ENGINEERING FOR ASSISTANCE. } \\
\text { THIS CIRCUIT IS PROPRIETARY TO LINEAR TECHNOLOGY AND } \\
\text { SUPPLIED FOR USE WITH LINEAR TECHNOLOGY PARTS. }\end{array}$ & \multicolumn{2}{|c|}{ Thursday, November 15, 2007} & \multicolumn{2}{|c|}{ SCALE: } & FILENAME: & SHEET & 1 & OF & 1 \\
\hline
\end{tabular}


E Microcontroller Code 

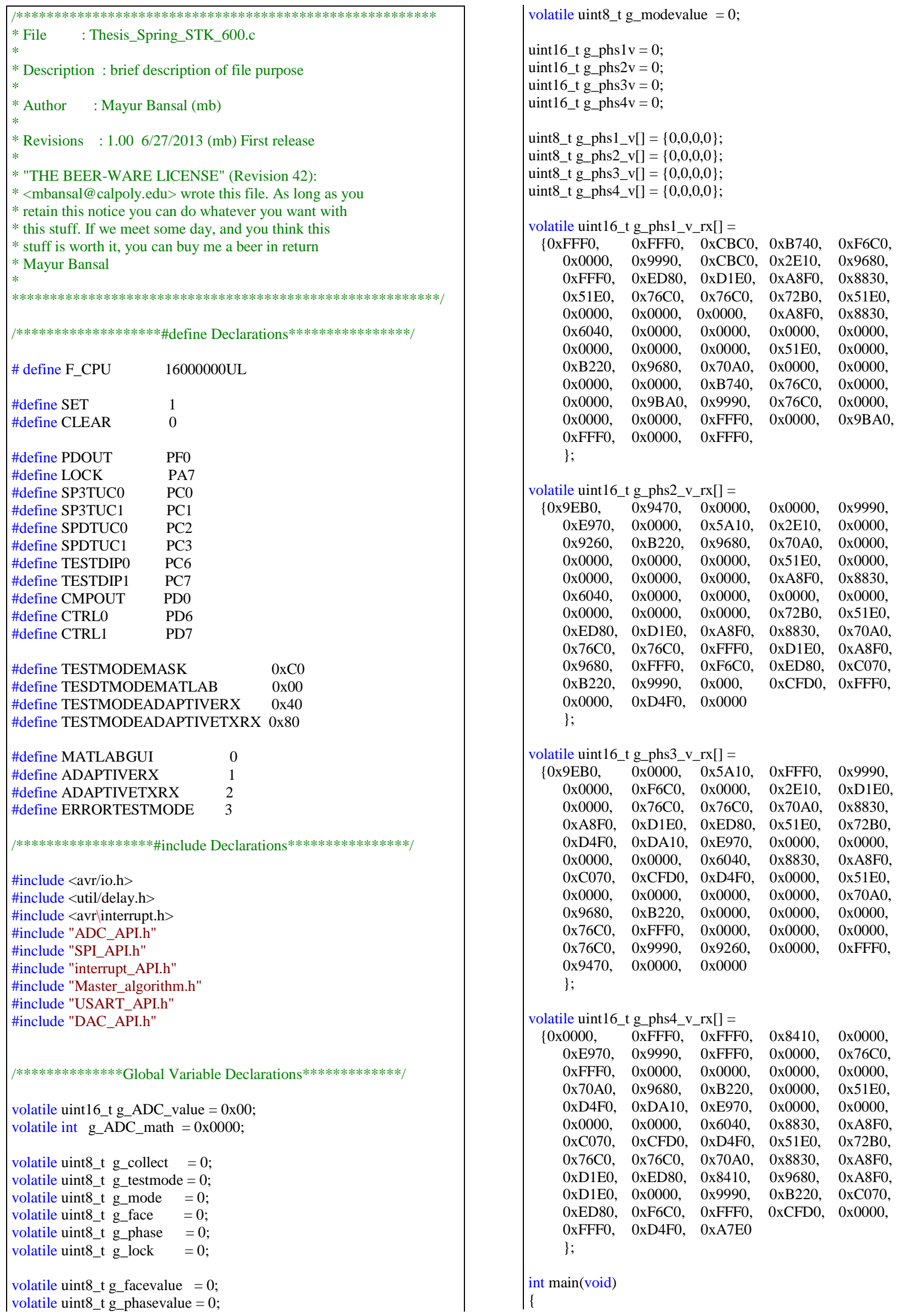
// Set the different I/O's

// set Lock as Output

DDRA $\mid=(1<<$ LOCK $)$;

// set peak detector output as input to ADC0

DDRA \& $=\sim(1<<$ PDOUT $)$;

// set the SPDT as outputs

DDRC $\mid=(1<<$ SPDTUC0 $) \mid(1<<$ SPDTUC1 $)$;

// set the SP3T as outputs

DDRC $\mid=(1<<$ SP3TUC0 $) \mid(1<<$ SP3TUC1 $)$;

$/ /$ set the TESTDIP as inputs

DDRC $\&=\sim(1 \ll$ TESTDIP0) $\& \sim(1 \ll$ TESTDIP1 $)$;

// set Comparator out as input

DDRD $\&=\sim(1<<$ CMPOUT $)$;

// set CTRL line as GPIO for RF board for future use.

//DDRD $\mid=(1<<$ CTRLO $) \mid(1<<$ CTRL 1$)$;

/* test CODE */

// Initialize all the Peripherals required

// Initialize ADC

initialize_ADC_single_conv();

set_ADC_clock () ;

enable_ADC();

set_ADC_trigger_source();

// Initialize SPI

SPI_MasterInit();

// Initialize USART

USART_init();

// disable the Semaphore

g_collect $=0$;

// Check to see what test mode the uC should be on

// MATLAB GUI mode

if $($ (PINC \& TESTMODEMASK $)==$ TESDTMODEMATLAB $)$

\{

SPI_MasterTransmit_DAC(set_pwrDACA,0xff,0xff);

while(1)

\{

// receive the mode

g_mode = USART_rx () ;

// receive the face

g_face = USART_rx();

// receive the phase 1 value

g_phs1_v[1] = USART_rx( $)$;

g_phs1_v[2] = USART_rx () ;

g_phs1_v[3] = USART_rx( $)$;

g_phs1_v[4] = USART_rx () ;

$/ /$ receive the phase 2 value

g_phs2_v[1] = USART_rx();

g_phs2_v[2] = USART_rx();

g_phs2_v[3] = USART_rx();

g_phs2_v[4] = USART_rx( $)$;

// receive the phase 3 value

g_phs3_v[1] = USART_rx();

g_phs3_v[2] = USART_rx ();

g_phs3_v[3] = USART_rx( $)$;

g_phs3_v[4] = USART_rx () ;

// receive the phase 4 value g_phs4_v[1] = USART_rx();

g_phs4_v[2] = USART_rx( $($;

g_phs4_v[3] = USART_rx () ;

g_phs4_v[4] = USART_rx ();
// convert the recive characters to number value g_mode = char_to_num(g_mode);

g_face $=$ char_to_num $($ g_face $)$;

// convert the recieve character phase value to voltage

value

g_phs $1 \mathrm{v}=$ convert_data_voltage (g_phs1_v);

g_phs2v = convert_data_voltage(g_phs2_v);

g_phs3v = convert_data_voltage(g_phs3_v);

g_phs $4 \mathrm{v}=$ convert_data_voltage $($ g_phs 4 _v $)$;

g_phs1v $=($ g_phs $1 \mathrm{v}<<4)$;

g_phs $2 \mathrm{v}=($ g_phs $2 \mathrm{v}<<4)$;

g_phs3v = (g_phs3v $<<4)$;

g_phs4v $=($ g_phs $4 \mathrm{v}<<4)$;

// set the system variables

set_face(g_face);

set mode(g mode);

g_phs1v);

SPI_MasterTransmit_DAC(setDACE,(g_phs1v >> 8),

g_phs2v);

g_phs3v);

SPI_MasterTransmit_DAC(setDACF,(g_phs2v >> 8),

SPI_MasterTransmit_DAC(setDACG,(g_phs3v >> 8),

SPI_MasterTransmit_DAC(setDACHpwrALL,(g_phs4v >> 8), g_phs4v);

\}

// Adaptive RX

else if $($ (PINC \& TESTMODEMASK $)==$

TESTMODEADAPTIVERX )

\{

// set the mode to receive

set_mode(RX);

// clear the semaphore

g_collect $=$ CLEAR;

// set the comparater refrence to $-50 \mathrm{dBm}$

SPI_MasterTransmit_DAC(set_pwrDACA,(MINPWRLVL >> 8), MINPWRLVL);

// Initialize Interrupts

enable_interrupts () ;

enable_int0_rising_edge();

while (1)

// semaphore check

if $\left(\mathrm{g} \_\right.$collect $==$CLEAR $)$

\{

// clear lock semaphore

g_lock = CLEAR;

clr_lock();

// start the face loop

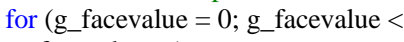

NUMBEROFFACES; g_facevalue++)

\{

set_face(g_facevalue+1);

// start the phase loop

for (g_phasevalue $=0 ;$ g_phasevalue $<$

NUMBEROFPOINTSRX; g_phasevalue++ )

SPI_MasterTransmit_DAC(setDACE,(g_phs1_v_rx[g_phasevalue ] >> 8), g_phs1_v_rx[g_phasevalue] ); 
SPI_MasterTransmit_DAC(setDACF,(g_phs2_v_rx[g_phasevalue] >> 8), g_phs2_v_rx[g_phasevalue] );

SPI_MasterTransmit_DAC(setDACG,(g_phs3_v_rx[g_phasevalue ] >> 8), g_phs3_v_rx[g_phasevalue] );

SPI_MasterTransmit_DAC(setDACHpwrALL,(g_phs4_v_rx[g_ph asevalue] > 8), g_phs4_v_rx[g_phasevalue] ); _delay_ms(DELAYTIME*20);

$$
\begin{aligned}
& \text { \} } \\
& \text { \} } \\
& \text { \} } \\
& \text { if }\left(\mathrm{g} \_ \text {collect }==\text { SET }\right) \\
& \{ \\
& \text { // set the lock semaphore } \\
& \text { g_lock = SET; } \\
& \text { set_lock(); }
\end{aligned}
$$

SPI_MasterTransmit_DAC(setDACE,(g_phs1_v_rx[g_phase] $>>$ 8), g_phs1_v_rx[g_phase] );

SPI_MasterTransmit_DAC(setDACF,(g_phs2_v_rx[g_phase] $>>$ 8), g_phs2_v_rx[g_phase] );

SPI_MasterTransmit_DAC(setDACG,(g_phs3_v_rx[g_phase] >> 8), g_phs3_v_rx[g_phase] );

SPI_MasterTransmit_DAC(setDACHpwrALL,(g_phs4_v_rx[g_ph ase] >> 8), g_phs4_v_rx[g_phase] );

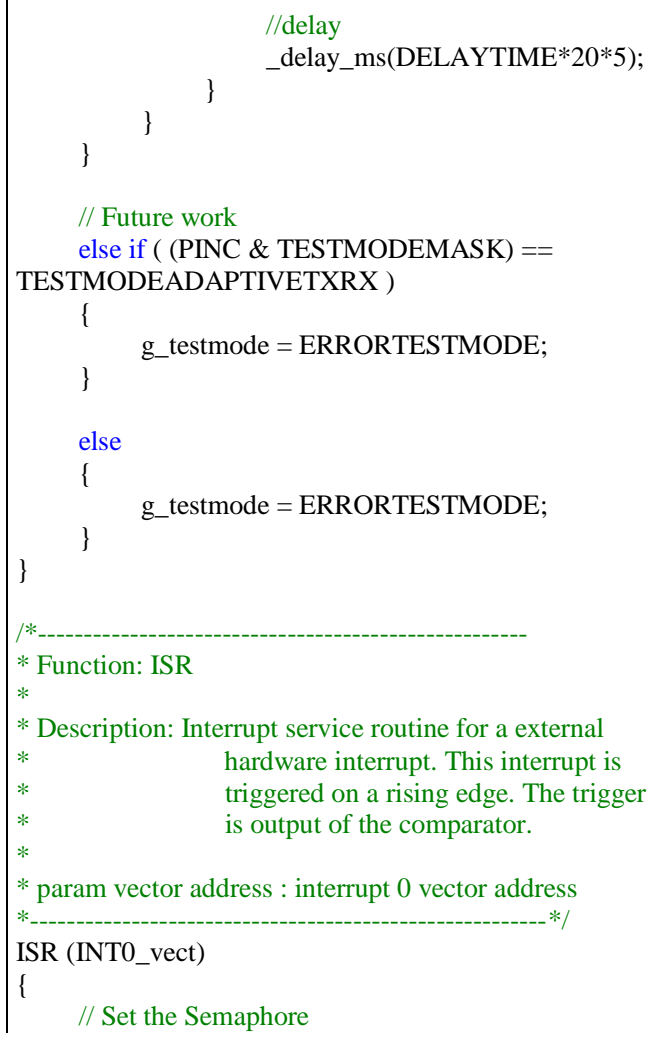

g_collect $=$ SET;

// Save the Important values

g_face = g_facevalue; // The face of Antenna used

g_phase = g_phasevalue; // The Phase index

if $(\mathrm{g}$ _lock $==$ CLEAR)

\{

start_conversion_ADC();

while(!(ADCSRA \& 0x10));

ADCSRA $\mid=(1<<$ ADIF $)$;

g_ADC_value $=$ ADC;

SPI_MasterTransmit_DAC(set_pwrDACA,(g_ADC_value

>> 8), g_ADC_value);

\}

else

\{

SPI_MasterTransmit_DAC(set_pwrDACA, (MINPWRLVL >> 8), MINPWRLVL);

\}

\}

/ $* * * * * * * * * * * * * * * * * * * * * * * * * * * * * * * * * * * * * * * * * * * * * * * * * * * * * * * * * * *$

* File : ADC_API.h

* Description : brief description of file purpose

$*$

* Author : Mayur Bansal (mb)

*

* Revisions : 1.00 6/27/2013 (mb) First release

*

* "THE BEER-WARE LICENSE" (Revision 42):

*<mbansal@calpoly.edu> wrote this file. As long as you

* retain this notice you can do whatever you want with

* this stuff. If we meet some day, and you think this

* stuff is worth it, you can buy me a beer in return

* Mayur Bansal

$*$

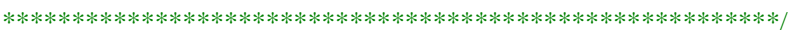

\#ifndef ADC_API_H_

\#define ADC_API_H_

$/ * * * * * * * * * * * * * * * * * * *$ Function Prototypes $* * * * * * * * * * * * * * * * * * /$

$/ *$

* Function: initialize_ADC_single_conv

$*$

* Description: Initialize the ADC

void initialize_ADC_single_conv();

* Function: enable_ADC

*

* Description: enable the ADC

(

void enable_ADC();

$/ *$

* Function: start_conversion_ADC

* Description: start a single conversation of the ADC

void start_conversion_ADC();

/*

* Function: set_ADC_clock

$*$

* Description: set the ADC clock to OSC/4

*-------------------------------------------------*/

void set_ADC_clock();

$/ *$ 


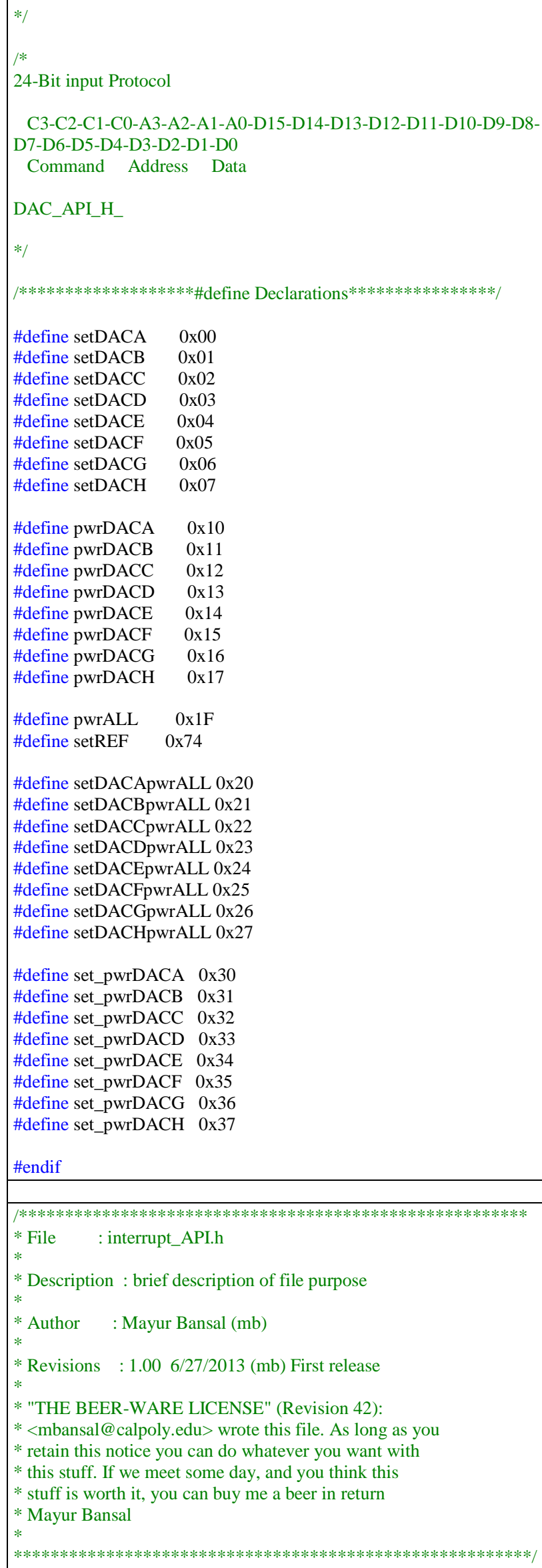

\#ifndef INTERRUPT_API_H \#define INTERRUPT_API_H

/ $* * * * * * * * * * * * * * * * * * * *$ Function Prototypes********************** 


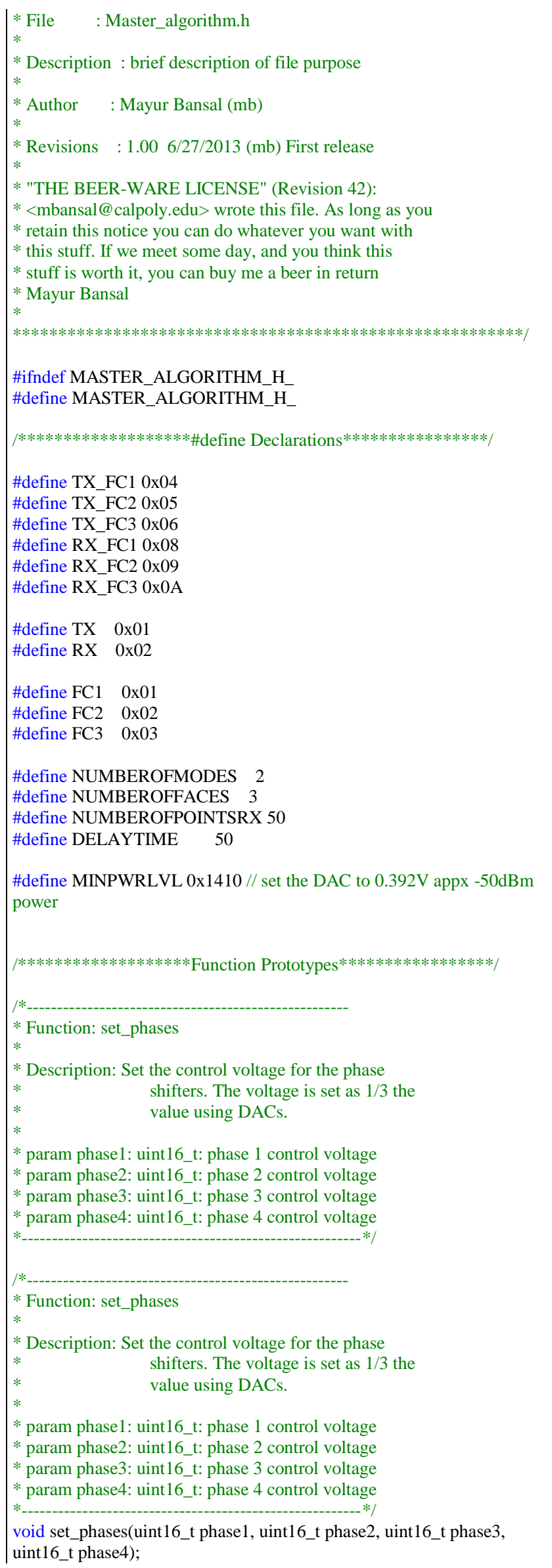

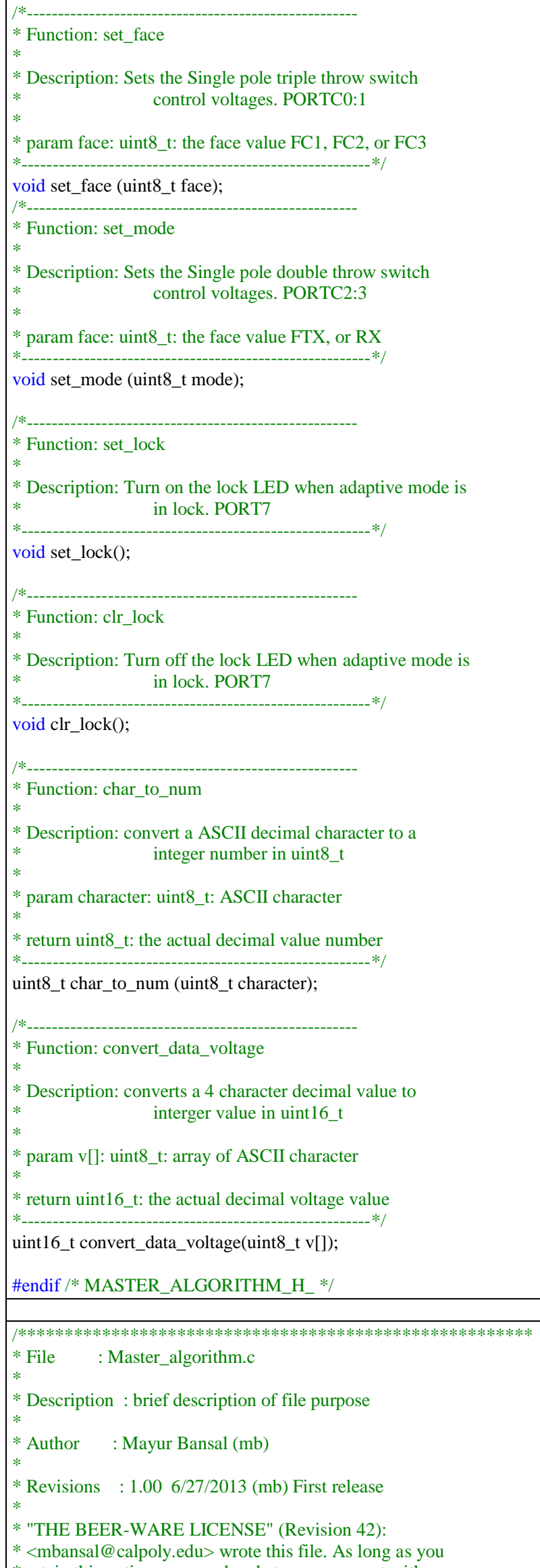




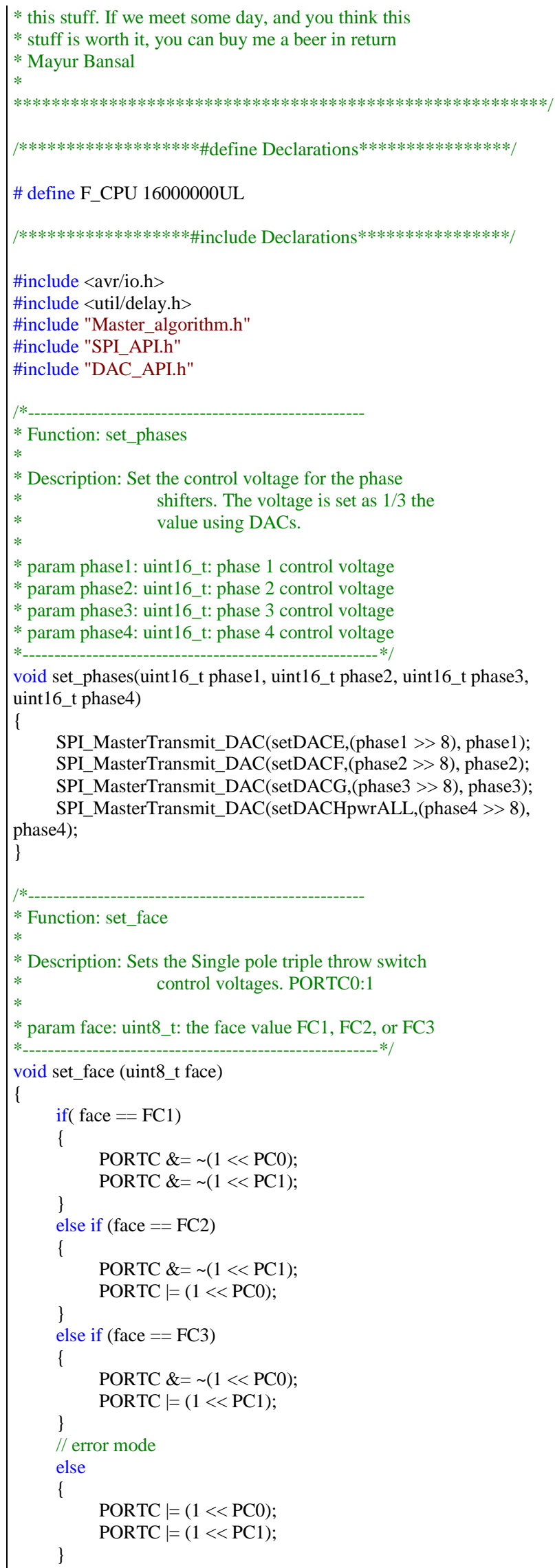




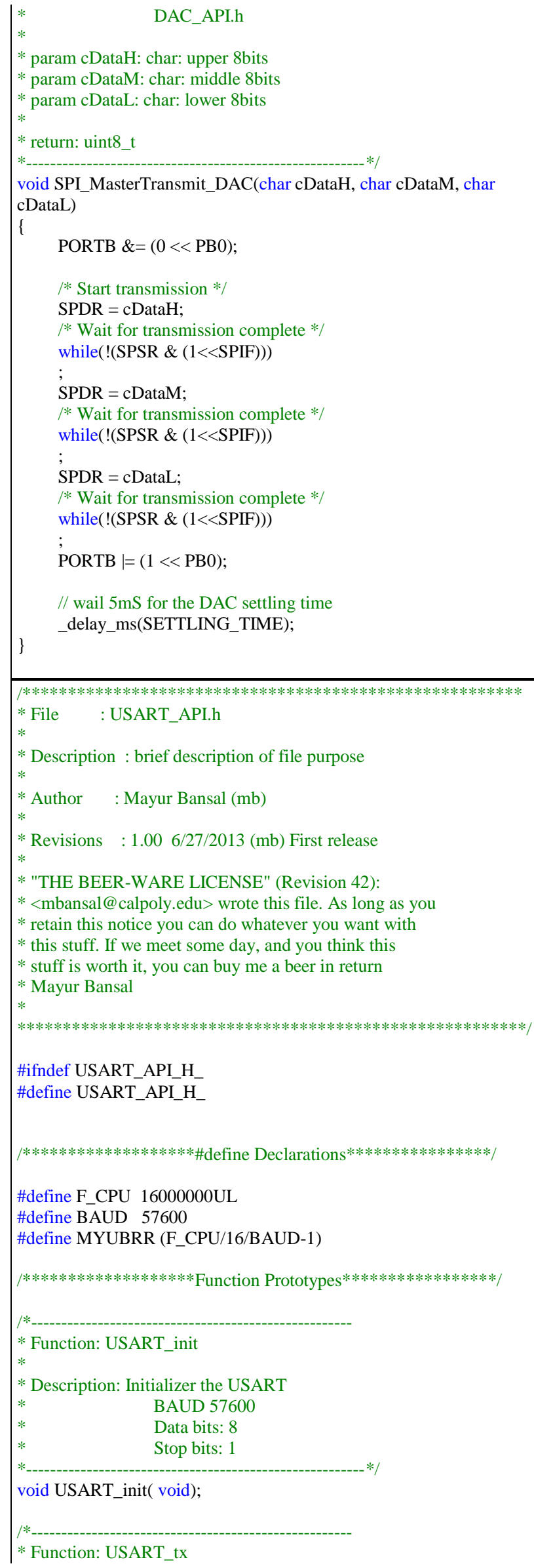

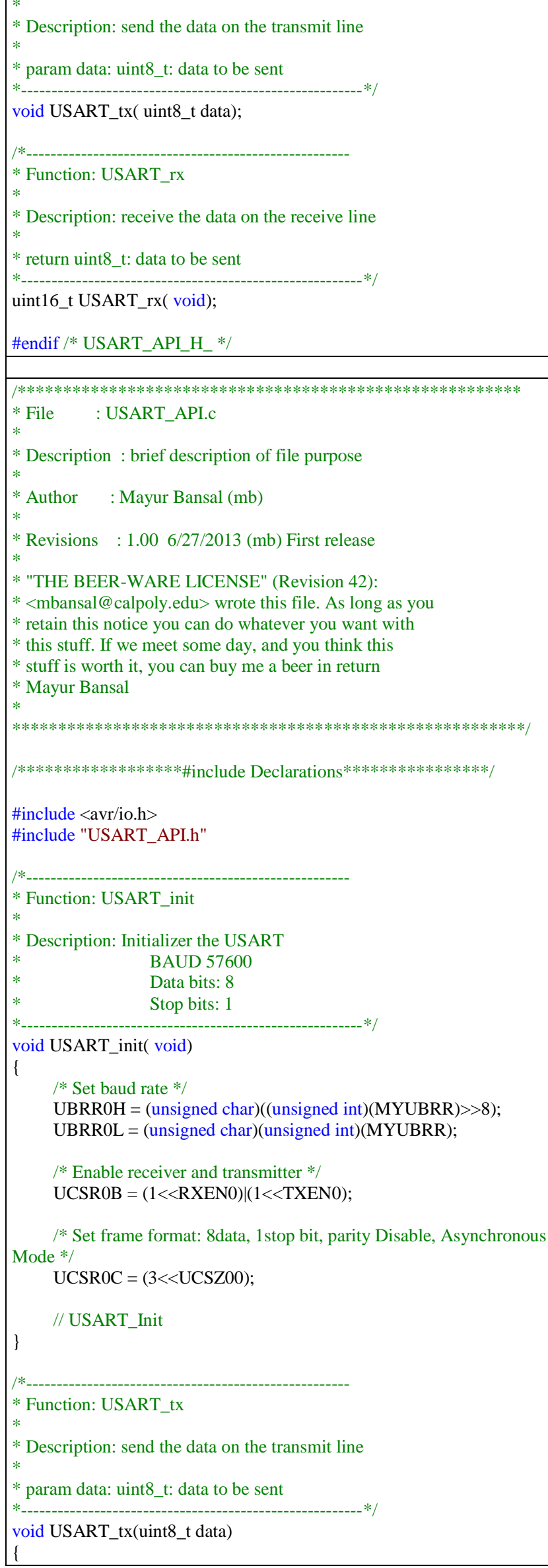


/* Wait for empty transmit buffer */ while ( ! UCSR0A \& (1<<UDRE0)) )

/* Put data into buffer, sends the data */ UDR0 = data;

\}

|*---------------------------------------------------

* Function: USART_rx

* Description: receive the data on the receive line

* return uint8_t: data to be sent

uint16_t USART_rx( void)

\{

$/ *$ Wait for data to be received */ while (!(UCSR0A \& $(1<<\mathrm{RXC} 0)))$

;

$/ *$ Get and return received data from buffer */ return UDR0; 


\section{F PCB Schematic}




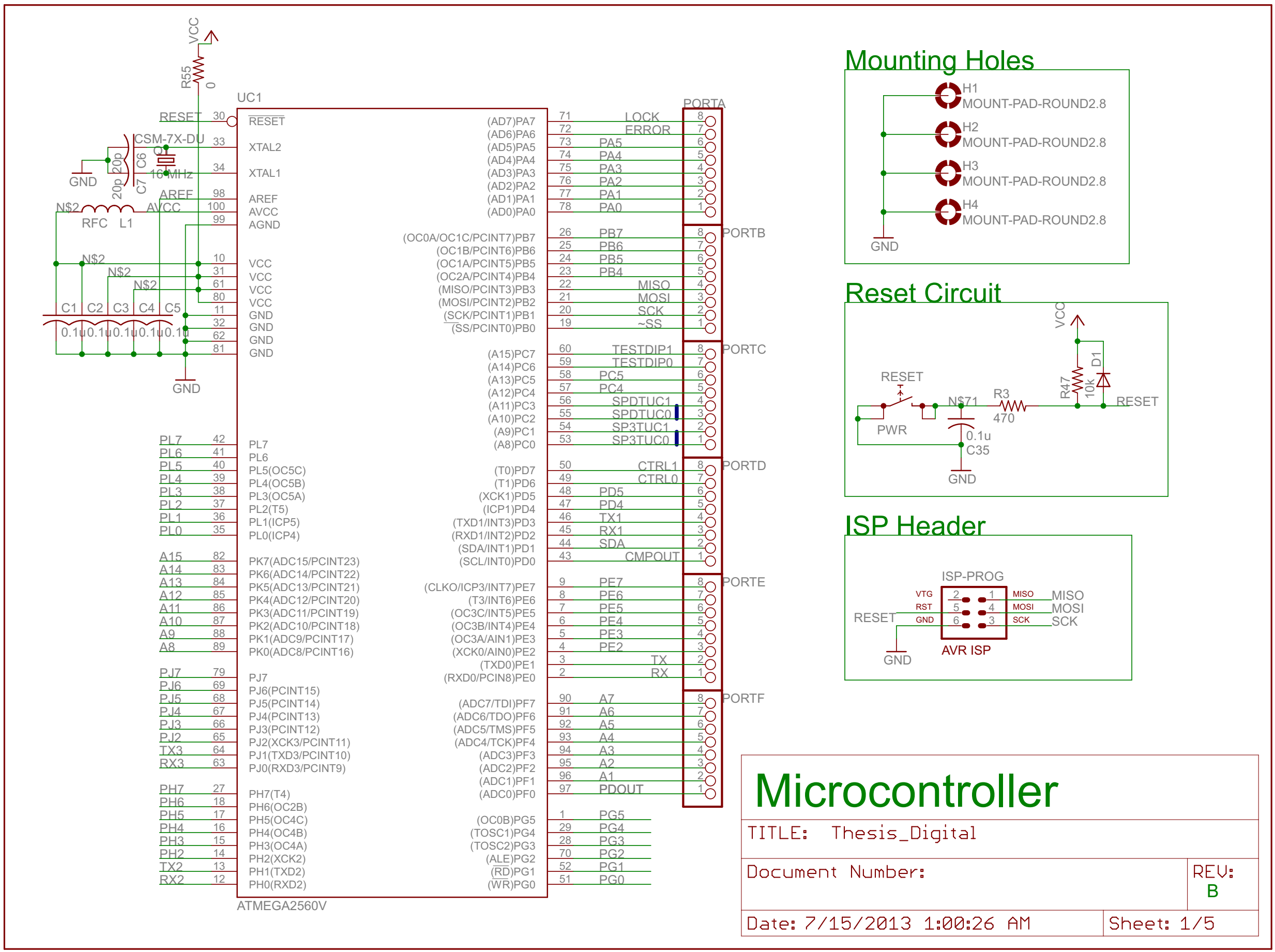




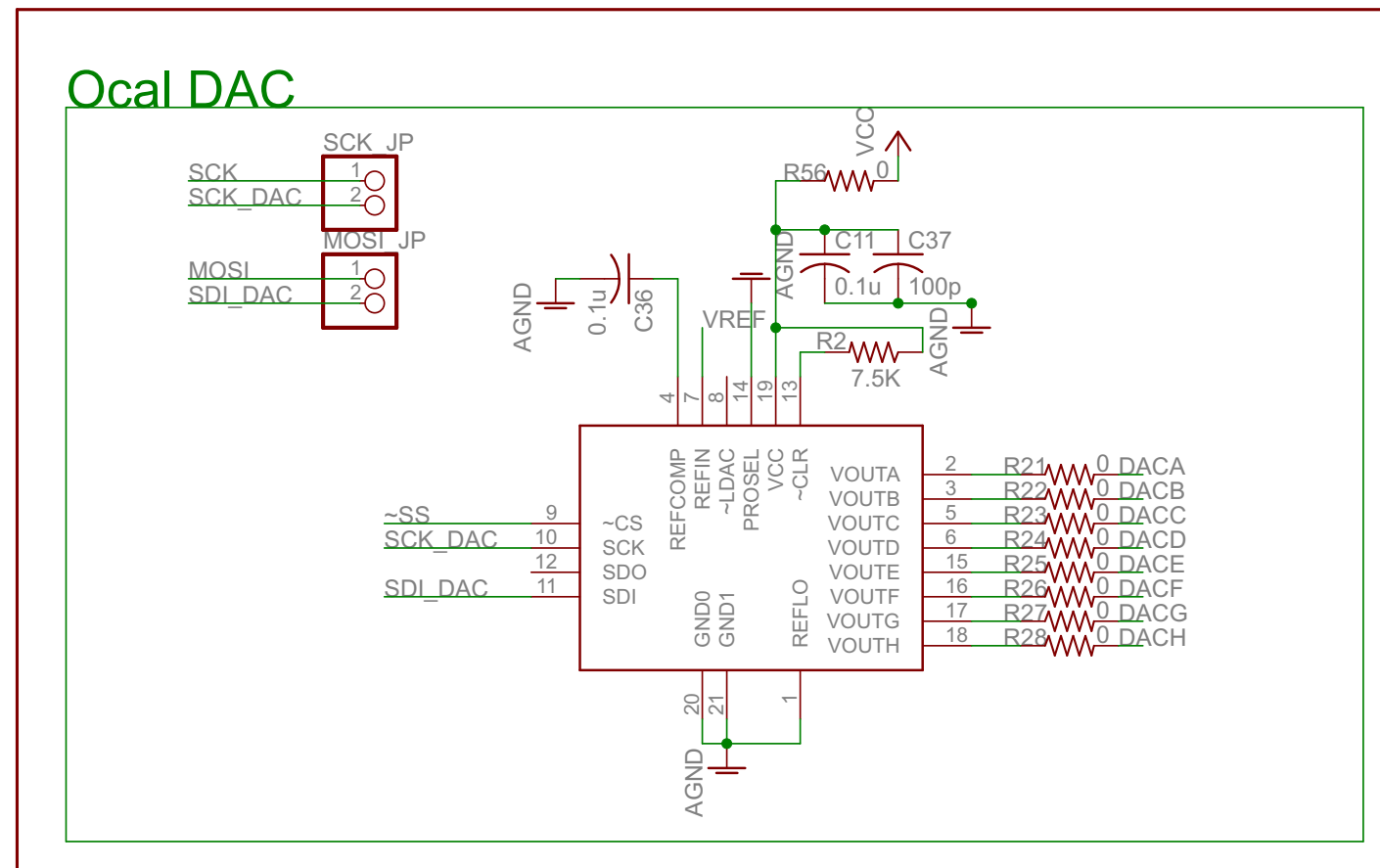

Phase Shifter Control line Amplifier
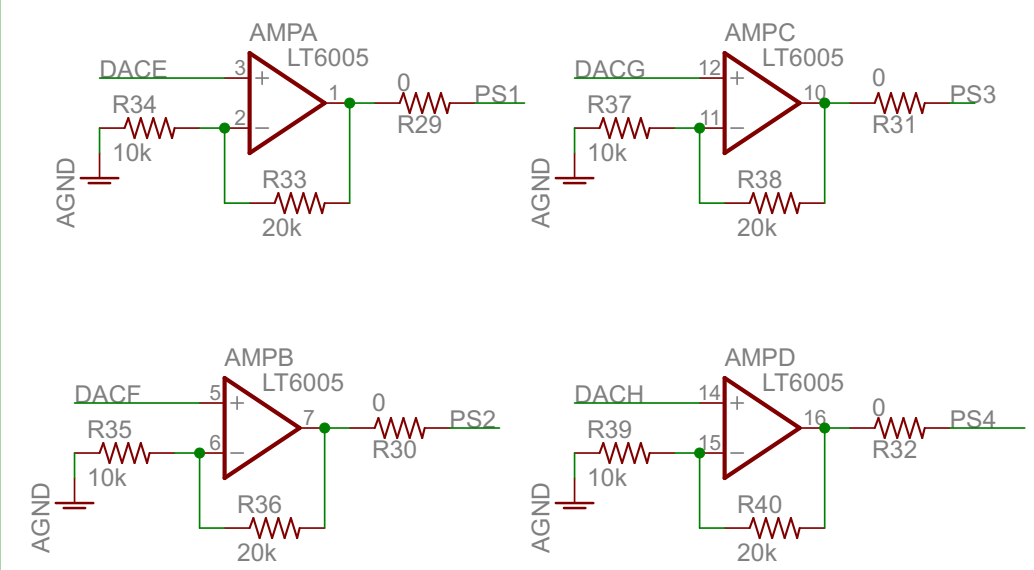

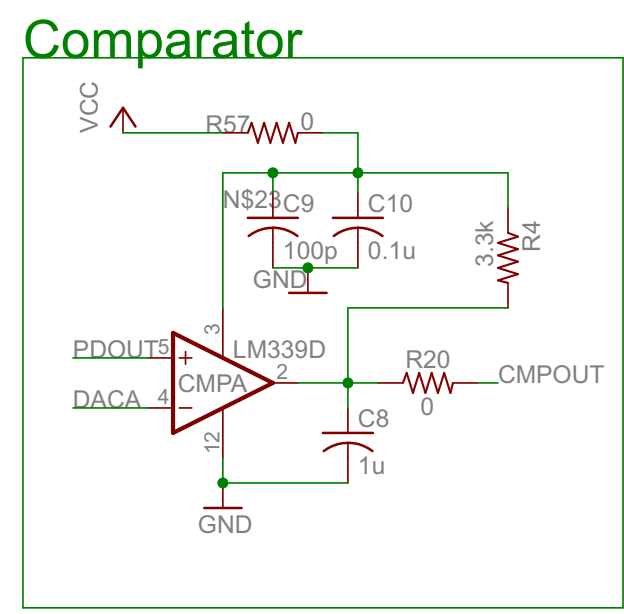

\section{Peak Detector}

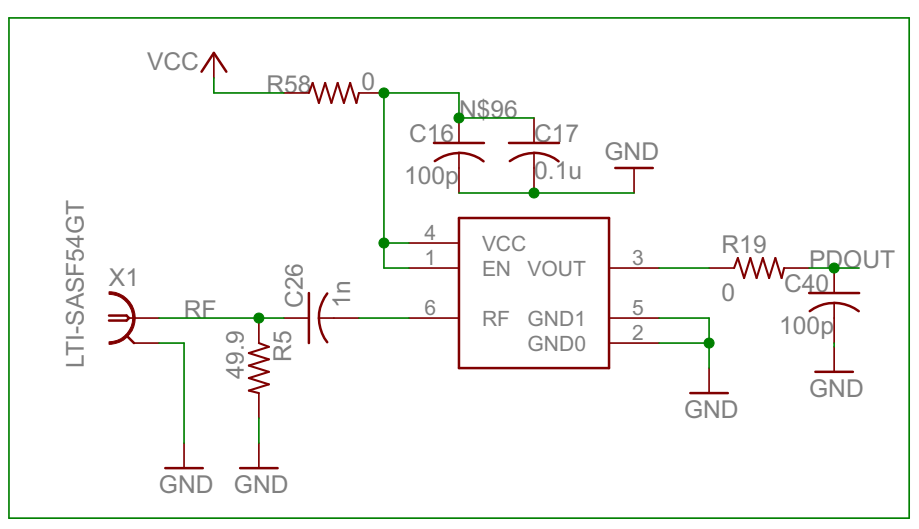

\section{Peripherals}

TITLE: Thesis_Digital

Document Number: 


\section{Input Voltage}

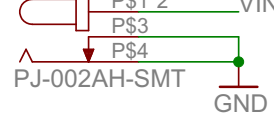

\section{V System}

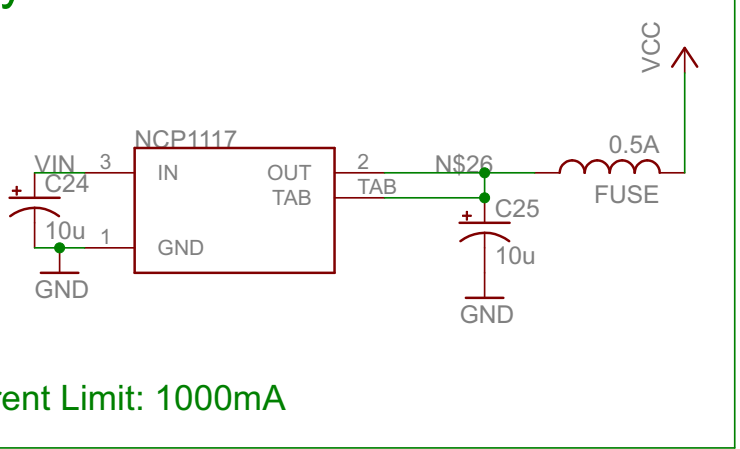

15 Volt Amplifier Rails

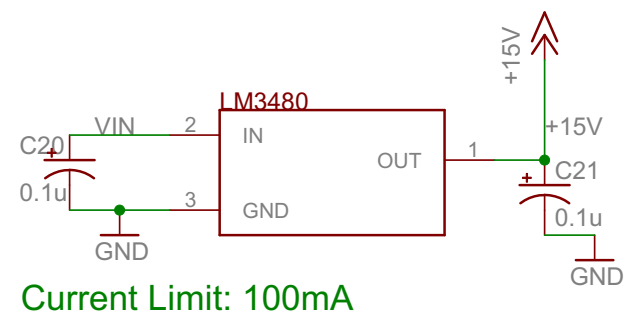

\subsection{DAC Reference}

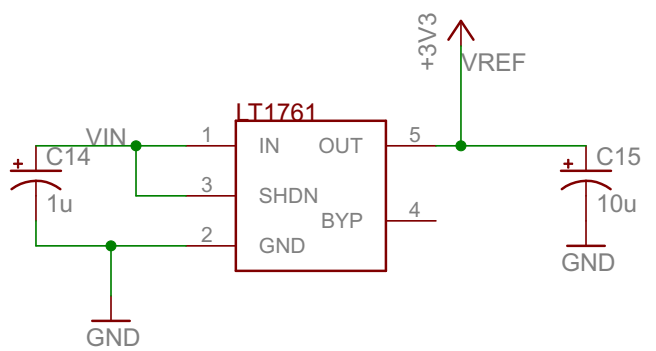

Current Limit: 100mA

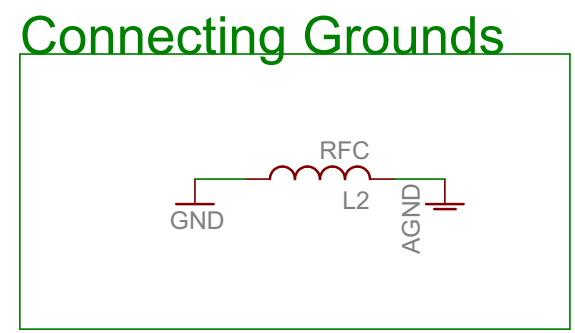

\section{Power}

TITLE: Thesis_Digital

Document Number: 


\section{Control Lines}

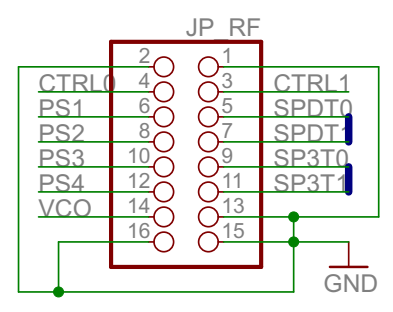

\section{Status LED}

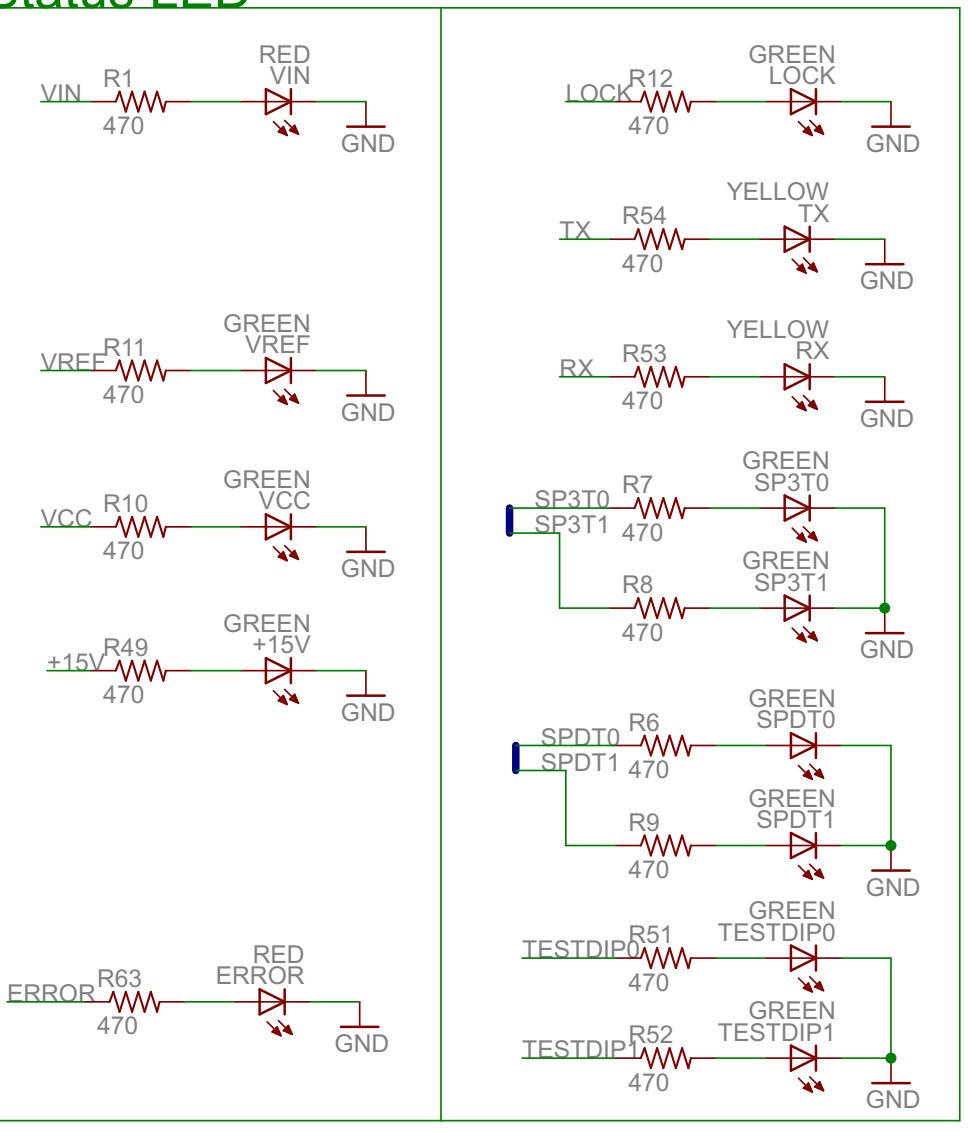

Signal Strength Display

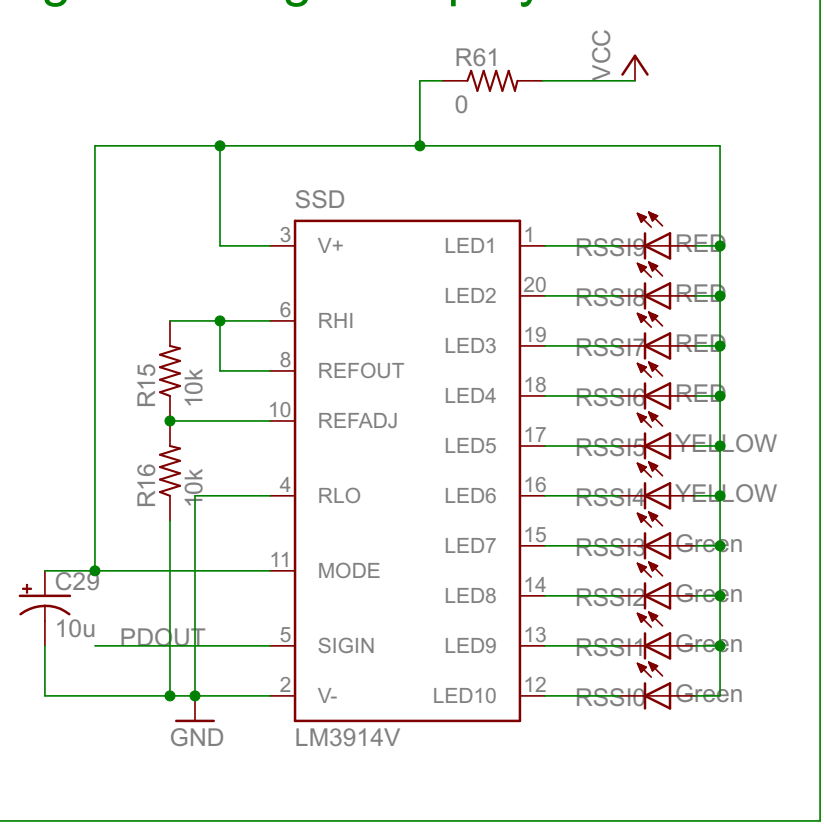

VCO Tunning

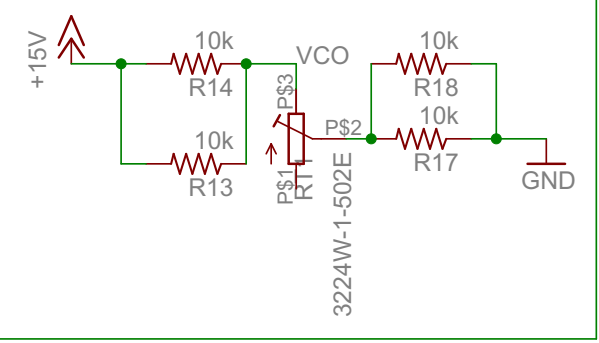

\section{RF Interface}

TITLE: Thesis_Digital

Document Number: 
RS-232 DRIVER

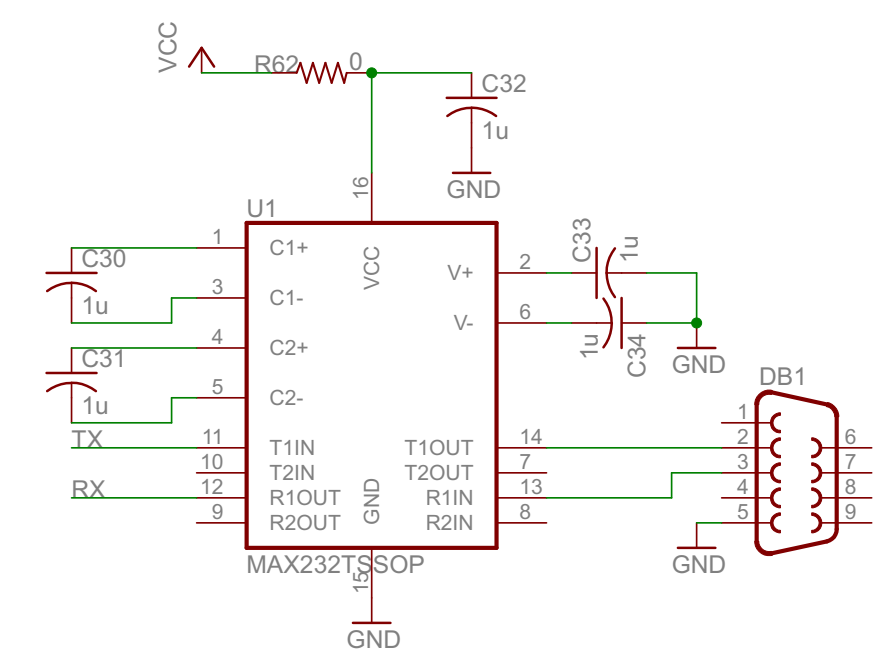

\section{ANT MODE}

\begin{tabular}{c|c|c} 
SPDT[1] & SPDT[0] & MODE \\
\hline 0 & 1 & RX \\
1 & 0 & TX
\end{tabular}

\section{ANT FACE}

\begin{tabular}{c|c|c} 
SP3T[1] & SP3T[0] & FACE \\
\hline 0 & 0 & 1 \\
0 & 1 & 3 \\
1 & 0 & 2
\end{tabular}

\section{TEST MODE}

\section{DIP Pins}

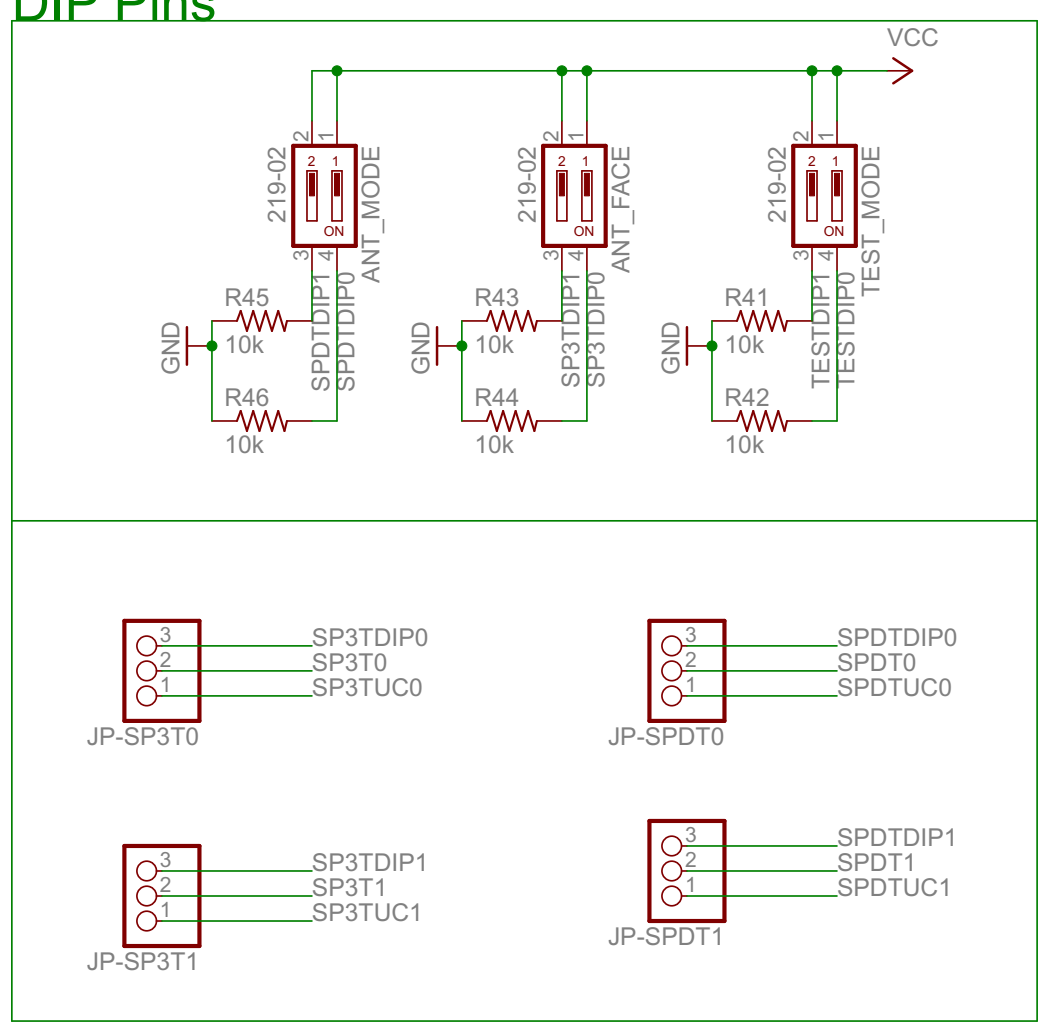

\begin{tabular}{c|c|l} 
TEST[1] & TEST[0] & MODE \\
\hline 0 & 0 & MATLAB GUI \\
0 & 1 & ADAPTIVE RX \\
1 & 0 & ADAPTIVE TX/RX \\
1 & 1 & UNUSED
\end{tabular}

\section{TEST MODE}

TITLE: Thesis_Digital

Document Number: 
G PCB Layout 


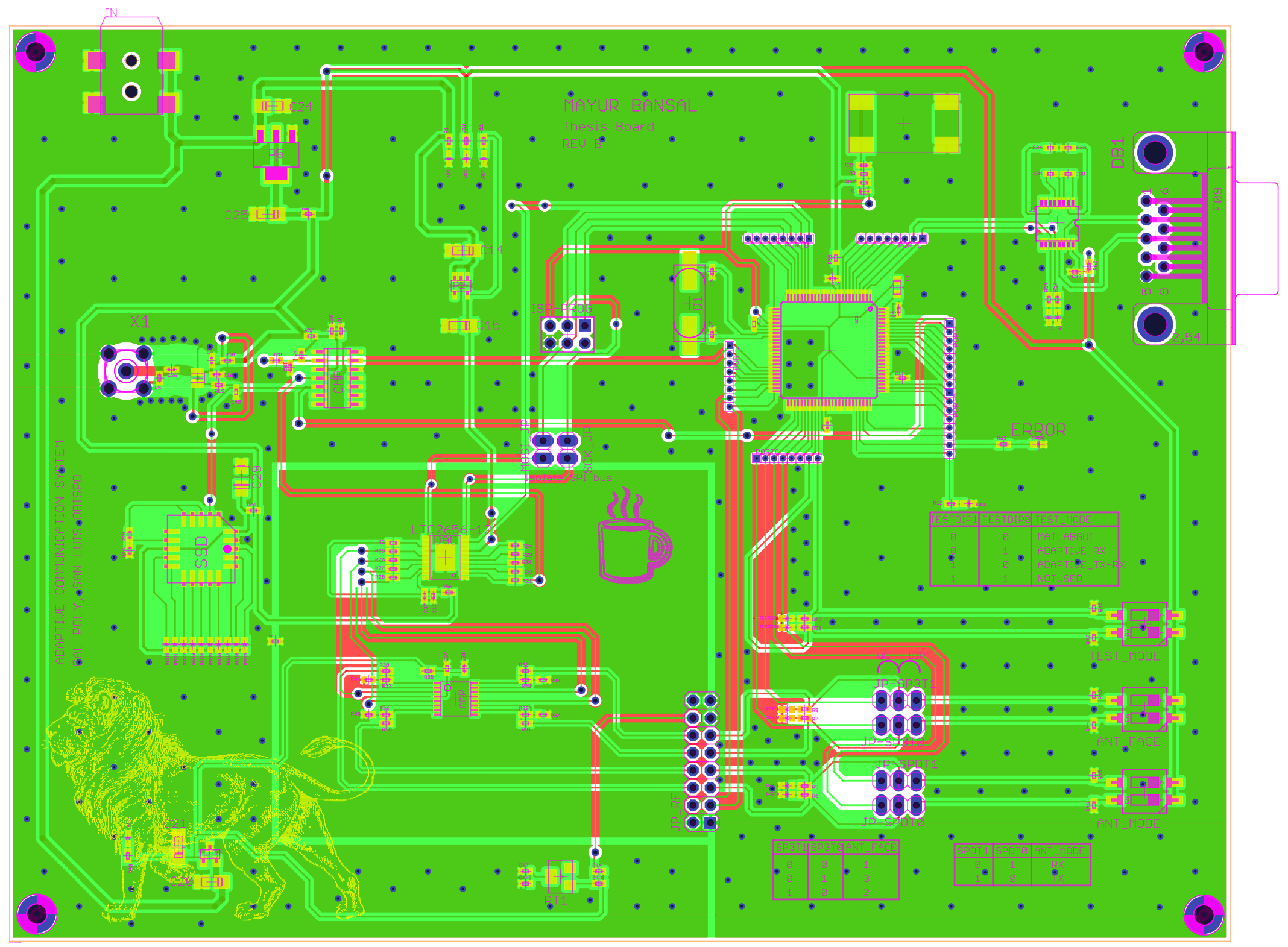




\section{H Bill Of Materials}

The complete electrical bill of materials (BOM) is presented below for the Digital Control Board. 


\begin{tabular}{|c|c|c|c|c|c|c|}
\hline Part & Manufacturer & Distributor & Model Number & Unit Price (\$) & Qty/Board & Total \\
\hline Crystal & ECS Inc & Digikey & ECS-160-20-5PXDU-TR & $\$ 1.46$ & \begin{tabular}{|r|}
1 \\
\end{tabular} & $\$ 1.46$ \\
\hline Microcontroller (ATmega2560) & Atmel & Digikey & ATMEGA2560-16AU & $\$ 18.19$ & 1 & $\$ 18.19$ \\
\hline LT1761(2.5V) & Linear Technology & Digikey & LT1761IS5-2.5\#TRMPBF & $\$ 2.62$ & 1 & $\$ 2.62$ \\
\hline NCP1117ST50T3G & ON Semi & Digikey & NCP1117ST50T3G & $\$ 0.49$ & 1 & $\$ 0.49$ \\
\hline LM3480(+15V) & Texas Instuments & Digikey & LM3480IM3-15/NOPB & $\$ 0.97$ & 1 & $\$ 0.97$ \\
\hline LTC2656 & Linear Technology & http://www.linear.com & LTC2656IFE-L12\#PBF & $\$ 14.37$ & 1 & $\$ 14.37$ \\
\hline LT5534 & Linear Technology & http://www.linear.com & LT5534ESC6\#TRMPBF & $\$ 6.50$ & 1 & $\$ 6.50$ \\
\hline LT6005 & Linear Technology & http://www.linear.com & LT6005CGN\#PBF & $\$ 2.06$ & 1 & $\$ 2.06$ \\
\hline LM339 & Texas Instuments & Digikey & LM339ADR & $\$ 0.41$ & 1 & $\$ 0.41$ \\
\hline LM3914 & Texas Instuments & Digikey & LM3914VX/NOPB & $\$ 3.66$ & 1 & $\$ 3.66$ \\
\hline MAX232 & Texas Instuments & Digikey & MAX232ECPW & $\$ 1.34$ & 1 & $\$ 1.34$ \\
\hline 16 Pin Conn (16 POS) & $\overline{\mathrm{FCI}}$ & Digikey & 67996-216HLF & $\$ 4.90$ & 2 & $\$ 9.80$ \\
\hline AVR-ISP-header (6POS) & FCI & Digikey & 67996-206HLF & $\$ 0.29$ & 6 & $\$ 1.74$ \\
\hline SPI Jumpers (2POS) & FCI & Digikey & 67996-104HLF & $\$ 0.27$ & 1 & $\$ 0.27$ \\
\hline DB-9 & TE Connectivity & Digikey & $1734351-1$ & $\$ 1.18$ & 1 & $\$ 1.18$ \\
\hline DIP- 2 POS & CTS Electrocomponents & Digikey & 219-2LPST & $\$ 0.60$ & 3 & $\$ 1.80$ \\
\hline Jumper & TE Connectivity & Digikey & $2-382811-1$ & $\$ 0.09$ & 4 & $\$ 0.36$ \\
\hline SMA & TE Connectivity & Digikey & $2081233-1$ & $\$ 1.65$ & 1 & $\$ 1.65$ \\
\hline 16 PIN IDC Cable & TE Connectivity & Digikey & A3DDH-1606G & $\$ 7.19$ & 1 & $\$ 7.19$ \\
\hline PWR70Q2S & C \& K Components & Digikey & PWR70Q2S & $\$ 3.89$ & 1 & $\$ 3.89$ \\
\hline LED Green & OSRAM Opto Semiconductors Inc & Digikey & LG Q971-KN-1 & $\$ 0.07$ & 16 & $\$ 1.09$ \\
\hline LED Red & OSRAM Opto Semiconductors Inc & Digikey & LS Q976-NR-1 & $\$ 0.09$ & 8 & $\$ 0.72$ \\
\hline LED Yellow & OSRAM Opto Semiconductors Inc & Digikey & LY Q976-P1S2-36 & $\$ 0.09$ & 3 & $\$ 0.27$ \\
\hline $0 \mathrm{Ohm}$ & Yageo & Digikey & RC0402JR-070RL & $\$ 0.01$ & 20 & $\$ 0.24$ \\
\hline $49.9 \mathrm{ohm}$ & Rohm Semiconductor & Digikey & MCR01MRTF49R9 & $\$ 0.10$ & 1 & $\$ 0.10$ \\
\hline $7.5 \mathrm{k}$ & Panasonic Electronic Components & Digikey & ERJ-2GEJ752X & $\$ 0.10$ & 2 & $\$ 0.20$ \\
\hline $3.3 \mathrm{k}$ & Panasonic Electronic Components & Digikey & ERJ-2GEJ332X & $\$ 0.10$ & 4 & $\$ 0.40$ \\
\hline $470 \mathrm{ohm}$ & Stackpole Electronics Inc & Digikey & RMCF0402JT470R & $\$ 0.02$ & 10 & $\$ 0.17$ \\
\hline $20 \mathrm{k}(0.5 \%)$ & Vishay Dale & Digikey & CRCW040220K0DHEDP & $\$ 0.28$ & 4 & $\$ 1.10$ \\
\hline $10 \mathrm{k}(0.5 \%)$ & Vishay Dale & Digikey & CRCW040210K0DHEDP & $\$ 0.28$ & 4 & $\$ 1.10$ \\
\hline $10 \mathrm{~K}$ & Panasonic Electronic Components & Digikey & ERJ-2RKF1002X & $\$ 0.10$ & 11 & $\$ 1.10$ \\
\hline $5 \mathrm{~K}$ POT & Bourns Inc. & Digikey & $3224 \mathrm{~W}-1-502 \mathrm{E}$ & $\$ 3.64$ & 1 & $\$ 3.64$ \\
\hline CER 0.1uF & Kemet & Digikey & C0402C104K8PACTU & $\$ 0.10$ & 9 & $\$ 0.90$ \\
\hline CER 20pF & Yageo & Digikey & CC0402JRNPO9BN200 & $\$ 0.10$ & 2 & $\$ 0.20$ \\
\hline CER 100pF 16V & Kemet & Digikey & C0402C101K4GACTU & $\$ 0.10$ & 4 & $\$ 0.40$ \\
\hline CER 1uF 16V & TDK Corporation & Digikey & C1005X5R1C105K050BC & $\$ 0.12$ & 7 & $\$ 0.84$ \\
\hline TANT $0.1 \mathrm{uF} 35 \mathrm{~V}$ & Kemet & Digikey & T491A104M035AT & $\$ 0.38$ & 2 & $\$ 0.76$ \\
\hline TANT 1uF 16V & Kemet & Digikey & T491A105K016AT & $\$ 0.34$ & 1 & $\$ 0.34$ \\
\hline TANT 10uF 20V & Kemet & Digikey & T494A106M020AT & $\$ 1.02$ & 3 & $\$ 3.06$ \\
\hline Fuse & TE Connectivity & Digikey & 0603SFF050F/32-2 & $\$ 0.35$ & 1 & $\$ 0.35$ \\
\hline RFC & TDK Corporation & Digikey & MLK1005S22NJ & $\$ 0.10$ & 2 & $\$ 0.20$ \\
\hline Diode & Micro Commercial Co & Digikey & 1N4148X-TP & $\$ 0.20$ & 1 & $\$ 0.20$ \\
\hline $20 \mathrm{~V}$ & CUI Inc & Digikey & ETSA200200UDC-P5P-SZ & $\$ 24.23$ & & $\$ 0.00$ \\
\hline Power Jack & CUI Inc & Digikey & PJ-002AH-SMT-TR & $\$ 1.89$ & 1 & $\$ 1.89$ \\
\hline
\end{tabular}




\section{Block Diagrams}

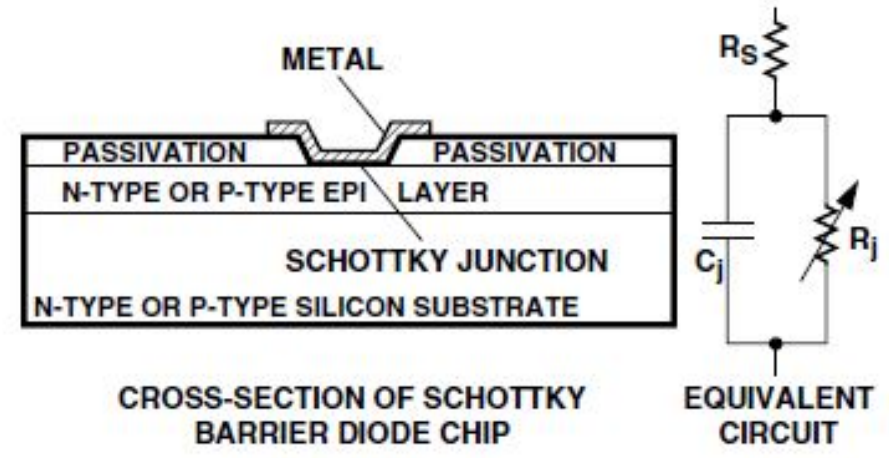

*SPICE model for HSMS-286x

(a) Electrical Model

*The parameters are for a single diode (HSMS-2860). Parameters also apply

*to the individual diodes within multiple diode configurations.

-subckt hsms286x Anode Cathode

D1 Anode Cathode DHOD

.MODEL DHOD D (IS=5E-8 CJO=0.18E-12 UJ=.65 BU=7 IBU=15E-5 EG=0.69 $\mathrm{N}=1.58 \mathrm{RS}=5 \mathrm{XTI}=2 \mathrm{M}=0.5)$

.ends hsms $286 x$

(b) Spice Netlist

Figure I.1: Schottky Diode Chip Model 


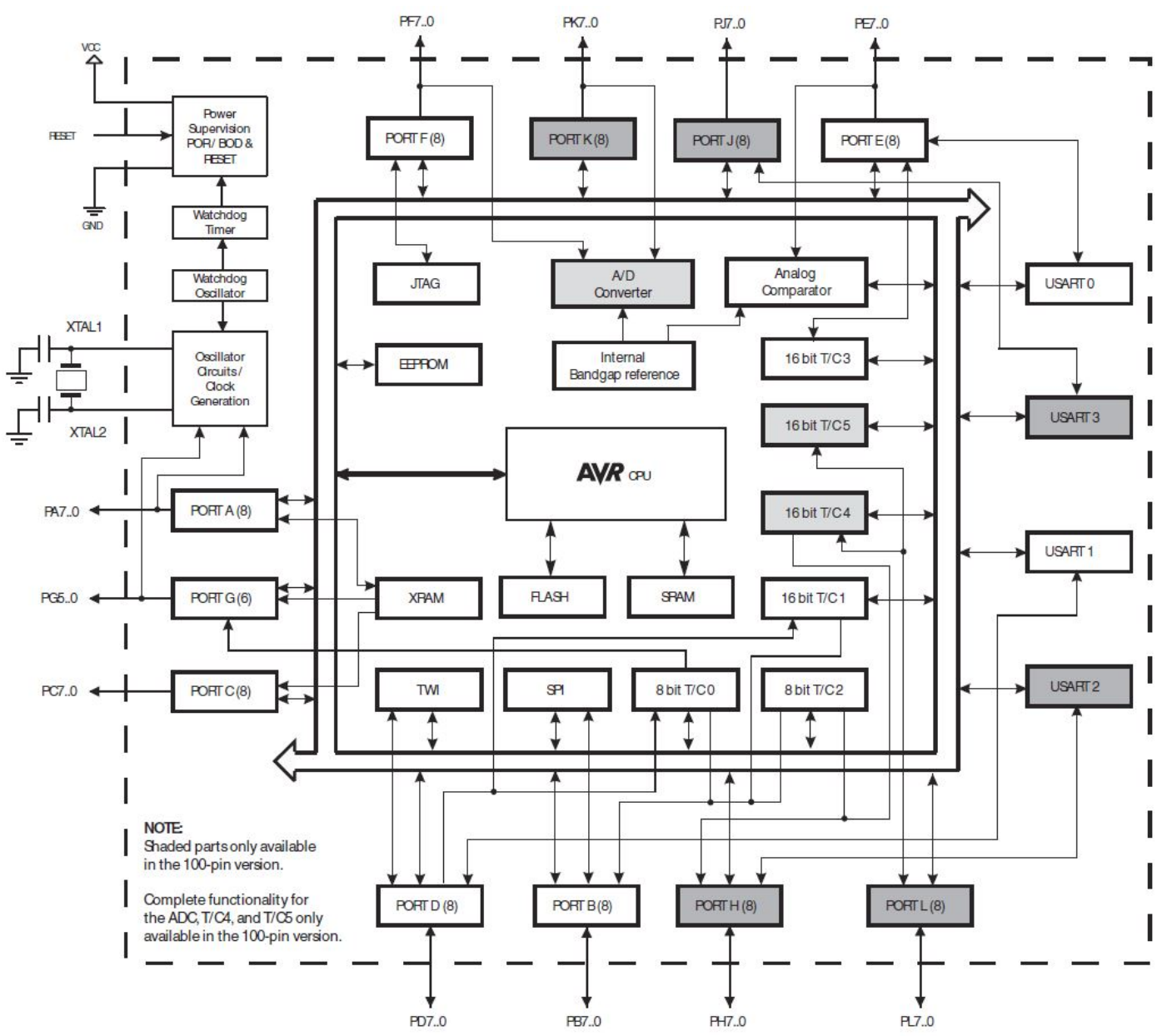

Figure I.2: ATmega2560 Block Diagram 


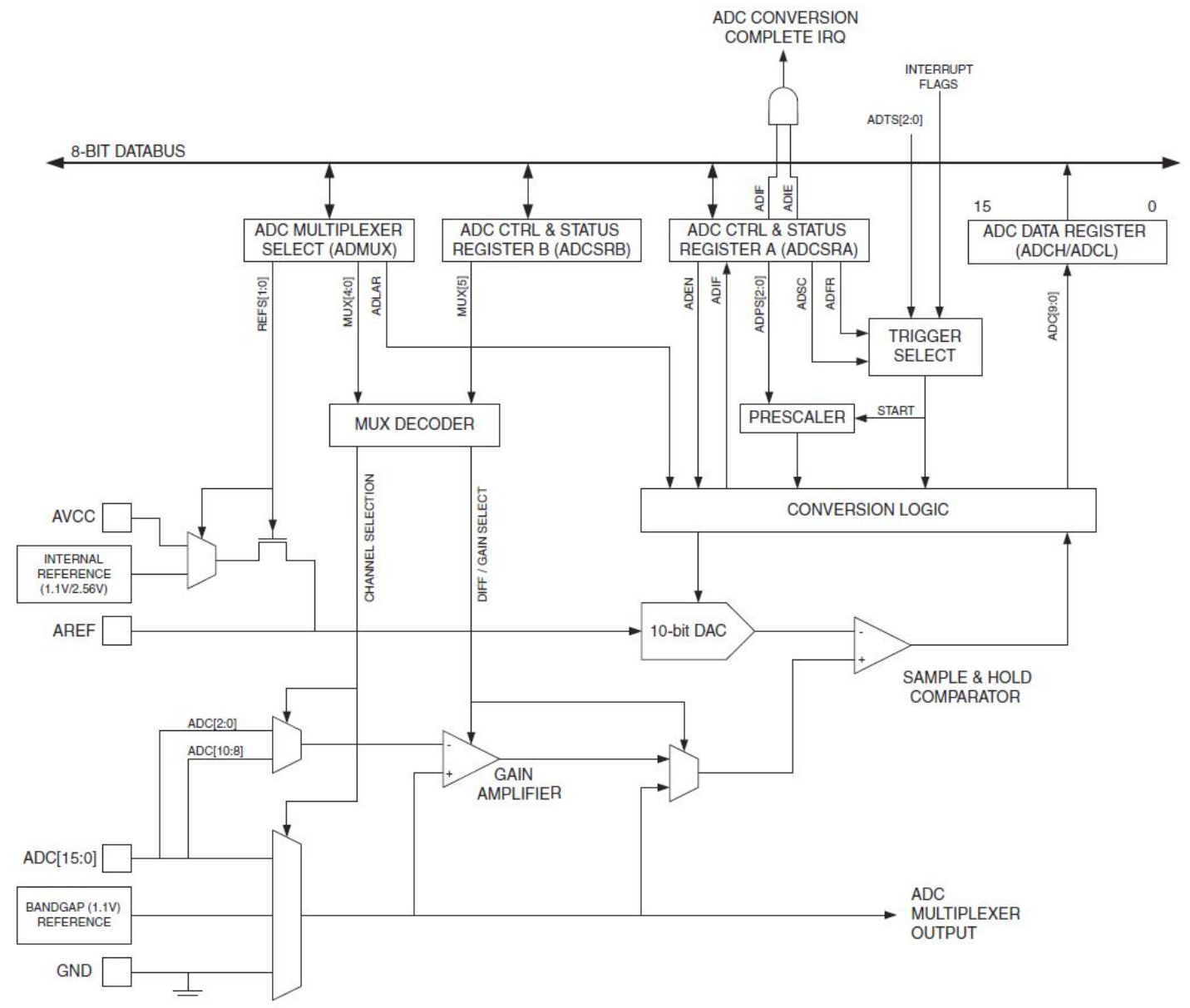

Figure I.3: Analog to Digital Converter Block Schematic 


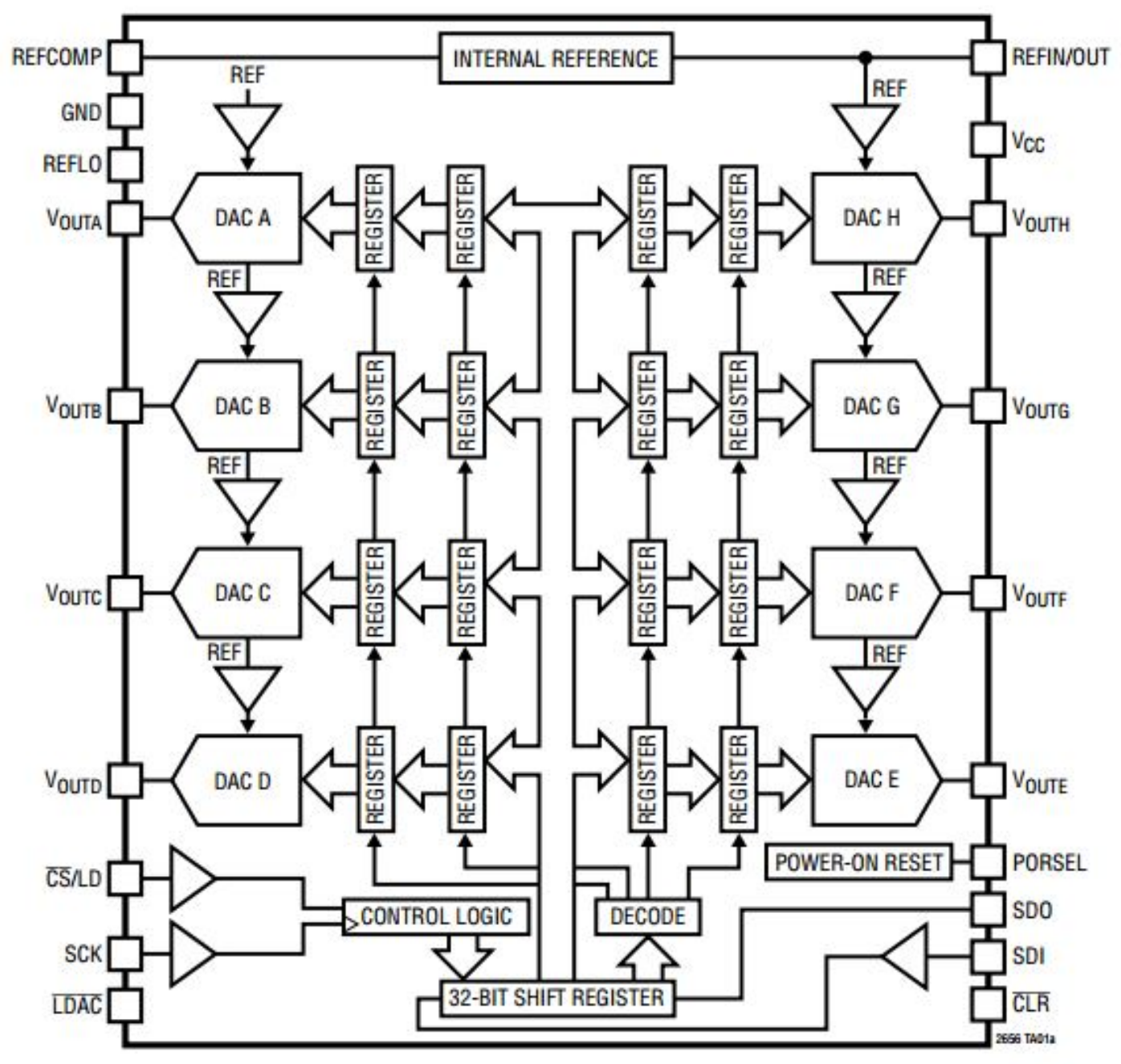

Figure I.4: LTC2656 Block Diagram 